\title{
Quantum optics with artificial atoms
}

\author{
ANTON Frisk KockUM
}

Applied Quantum Physics Laboratory Department of Microtechnology and Nanoscience CHALMERS UNIVERSITY OF TECHNOLOGY

Göteborg, Sweden 2014 
Quantum optics with artificial atoms

ANTON FRISK KOCKUM

ISBN 978-91-7597-113-1

(C)ANTON FRISK KOCKUM, 2014

Doktorsavhandlingar vid Chalmers tekniska högskola

Ny serie nr 3794

ISSN 0346-718X

Applied Quantum Physics Laboratory

Department of Microtechnology and Nanoscience - MC2

Chalmers University of Technology

SE-412 96 Göteborg, Sweden

Telephone +46 (0)317721000

www.chalmers.se

Author email: friska@chalmers.se

ISSN 1652-0769

Technical Report MC2-291

Cover: Clockwise from top left: a setup for microwave photon detection using cascaded three-level systems (Paper V); time evolution of the coherent state in a resonator, dispersively coupled to a qubit, conditioned on the qubit state (Paper I); a model for a giant multi-level atom coupled at several points (possibly wavelengths apart) to a $1 \mathrm{D}$ waveguide (Paper VII); zoom-in on part of a transmon qubit coupled to surface acoustic waves, realizing a giant atom (Paper VI).

Printed by Chalmers Reproservice

Göteborg, Sweden 2014 
Quantum optics with artificial atoms

ANTON FRISK KOCKUM

Applied Quantum Physics Laboratory

Department of Microtechnology and Nanoscience

Chalmers University of Technology

\begin{abstract}
Quantum optics is the study of interaction between atoms and photons. In the eight papers of this thesis, we study a number of systems where artificial atoms (here, superconducting circuits emulating the level structure of an atom) enable us to either improve on known concepts or experiments from quantum optics with natural atoms, or to explore entirely new regimes which have not been possible to reach in such experiments.

Paper I shows how unwanted measurement back-action in a parity measurement can be avoided by fully using the information in the measurement record. Paper III is a proof-of-principle experiment demonstrating that an artificial atom built from superconducting circuits can mediate a strong photon-photon interaction. In Papers II and V, we theoretically investigate whether this interaction can be used in a setup for detecting propagating microwave photons, making the photon to be detected impart a phase shift on a coherent probe signal. We find that one atom is not enough to overcome the quantum background noise, but it turns out that several atoms cascaded in the right way can do the trick.

In Paper IV, we explain experimental results for a driven artificial atom coupled to photons in a resonator. The last three papers all deal with an artificial atom coupled to a bosonic field at several points, which can be wavelengths apart. Paper VI is a ground-breaking experimental demonstration of coupling between an artificial atom and propagating sound in the form of surface acoustic waves (SAWs). The short SAW wavelength makes the atom "giant" in comparison; the effects of this new regime is explored theoretically in Paper VII, where the multiple coupling points are shown to give interference effects affecting both the atom's relaxation rate and its energy levels. In Paper VIII, an artificial atom in front of a mirror is used to probe the mode structure of quantum vacuum fluctuations.
\end{abstract}

Keywords: Quantum optics, quantum stochastic calculus, quantum measurement, transmon, circuit QED, photon detector, parity measurement, surface acoustic waves, artificial atoms 



\section{List of publications}

This thesis is based on the work contained in the following eight papers, which are referred to in the text by their Roman numerals:

I. Undoing measurement-induced dephasing in circuit QED Anton Frisk Kockum, Lars Tornberg, and Göran Johansson Physical Review A 85, 052318 (2012)

II. Breakdown of the cross-Kerr scheme for photon counting Bixuan Fan, Anton Frisk Kockum, Joshua Combes, Göran Johansson, Io-Chun Hoi, C. M. Wilson, Per Delsing, G. J. Milburn, and Thomas M. Stace Physical Review Letters 110, 053601 (2013)

III. Giant cross-Kerr effect for propagating microwaves induced by an artificial atom

Io-Chun Hoi, Anton Frisk Kockum, Tauno Palomaki, Thomas M. Stace, Bixuan Fan, Lars Tornberg, Sankar R. Sathyamoorthy, Göran Johansson, Per Delsing, and C. M. Wilson

Physical Review Letters 111, 053601 (2013)

IV. Detailed modelling of the susceptibility of a thermally populated, strongly driven circuit-QED system

Anton Frisk Kockum, Martin Sandberg, Michael R. Vissers, Jiansong Gao, Göran Johansson, and David P. Pappas

Journal of Physics B: Atomic, Molecular and Optical Physics 46, 224014 (2013) 
V. Quantum nondemolition detection of a propagating microwave photon

Sankar R. Sathyamoorthy, Lars Tornberg, Anton Frisk Kockum, Ben Q. Baragiola, Joshua Combes, C. M. Wilson, Thomas M. Stace, and Göran Johansson

Physical Review Letters 112, 093601 (2014)

VI. Propagating phonons coupled to an artificial atom

Martin V. Gustafsson, Thomas Aref, Anton Frisk Kockum, Maria K.

Ekström, Göran Johansson, and Per Delsing

Science 346, 207 (2014)

VII. Designing frequency-dependent relaxation rates and Lamb shifts for a giant artificial atom

Anton Frisk Kockum, Per Delsing, and Göran Johansson

Physical Review A 90, 013837 (2014)

VIII. Probing the quantum vacuum with an atom in front of a mirror

Io-Chun Hoi, Anton Frisk Kockum, Lars Tornberg, Arsalan Pourkabirian, Göran Johansson, Per Delsing, and C. M. Wilson Submitted (2014). ArXiv preprint: 1410.8840 


\section{Acknowledgements}

First of all I would like to thank my supervisor Göran Johansson, for giving me the chance to work in the fascinating field of quantum optics with artificial atoms.

I owe big thanks to Lars Tornberg. You taught me the basics when I started out as a master student, and then returned to impart more knowledge to me when I continued as a $\mathrm{PhD}$ student. I really appreciate how you always were available for fun discussions about physics and climbing.

Thanks also to all the rest of the people in the Applied Quantum Physics Laboratory, and also in the Quantum Device Physics Laboratory, for making this such a fun and stimulating place to work.

I want to thank Bixuan Fan, Thomas Stace, Gerard Milburn, and the rest of the group in Brisbane, for hosting me during my visit to University of Queensland. I also want to thank my colleagues in Delft, Waterloo, Albuquerque, and Boulder for fruitful collaborations.

To Birger Jörgensen, and all the other great teachers who helped me reach this point.

Thanks to Göran, Anna, Lars, Thomas, and Per for your helpful comments on the drafts of this thesis.

Thank you, friends and family, for your support and for all the good times we have shared.

Göteborg, November 2014 Anton Frisk Kockum 



\section{Contents}

List of publications $\quad$ V

Acknowledgements

$\begin{array}{lll}\text { Contents IX } & \text { IX }\end{array}$

List of figures $\quad$ XIII

$\begin{array}{ll}\text { Nomenclature } & \text { XV }\end{array}$

1 Introduction 1

1.1 Quantum optics in superconducting circuits . . . . . . . 2

1.2 Reaching new regimes with artificial atoms . . . . . 5

1.3 Quantum fluctuations of the vacuum . . . . . . . . 7

1.4 Quantum computing and parity measurement . . . . . . 9

1.5 Overview of the thesis . . . . . . . . . . . 12

2 Artificial atoms and 1D waveguides 15

2.1 Circuit QED . . . . . . . . . . . . . . 16

2.2 The quantized transmission line . . . . . . . . . . 18

2.2.1 The infinite $1 \mathrm{D}$ waveguide . . . . . . . . . . 18

2.2 .2 Mirrors and resonators . . . . . . . . . . . . . . . 21

2.3 The transmon qubit . . . . . . . . . . . . . . 22

2.4 Surface acoustic waves . . . . . . . . . . . 25

2.4.1 Classical SAW theory . . . . . . . . . . . . 25

2.4.2 Coupling SAWs to a transmon . . . . . . . . . . . 32

2.5 The Jaynes-Cummings model . . . . . . . . . . . . . . 35 
3 Master equations and input-output theory 41

3.1 Master equation for an open quantum system . . . . . . . . 42

3.2 Input-output theory . . . . . . . . . . . . . . . . . 45

3.3 Quantum stochastic calculus . . . . . . . . . . 47

3.4 Fock-state input . . . . . . . . . . . . . 50

3.4.1 Deriving the coupled master equations . . . . . . . 51

3.4.2 Example - a photon meeting an atom ....... 54

4 Quantum measurements $\quad 57$

4.1 Measurement back-action and SMEs . . . . . . . . . . 57

4.1.1 An example of back-action . . . . . . . . . 58

4.1.2 Time evolution of the density matrix . . . . . . . . 59

4.2 Photon detection . . . . . . . . . . . . . . 60

4.3 Homodyne detection . . . . . . . . . . . . . 63

4.4 Susceptibility . . . . . . . . . . . . . . . 66

5 Cascaded quantum systems $\quad 69$

5.1 The $(S, L, H)$ formalism $\ldots \ldots \ldots \ldots$

$5.2(S, L, H)$ rules and examples $\ldots \ldots \ldots \ldots \ldots$

5.2.1 Series product, concatenation, and feedback . . . . 71

5.2.2 Example 1 - coupled cavities and a circulator . . . . 73

5.2 .3 Example 2 - a giant artificial atom . . . . . . . . 77

6 Paper overview $\quad 81$

6.1 Paper I - Undoing measurement-induced dephasing in circuit QED . . . . . . . . . . . . . . . . 81

6.2 Paper II - Breakdown of the cross-Kerr scheme for photon counting . . . . . . . . . . . . . 83

6.3 Paper III - Giant cross-Kerr effect for propagating microwaves induced by an artificial atom . . . . . . . . . 8 85

6.4 Paper IV - Detailed modelling of the susceptibility of a thermally populated, strongly driven circuit-QED system . . . . 86

6.5 Paper V - Quantum nondemolition detection of a propagating microwave photon . . . . . . . . . 86

6.6 Paper VI - Propagating phonons coupled to an artificial atom 88

6.7 Paper VII - Designing frequency-dependent relaxation rates and Lamb shifts for a giant artificial atom . . . . . . . 89

6.8 Paper VIII - Probing the quantum vacuum with an atom in front of a mirror . . . . . . . . . . . . 90 
$\begin{array}{lll}7 & \text { Summary and outlook } & 93\end{array}$

$\begin{array}{ll}\text { A Transformations } & 97\end{array}$

A.1 Properties and identities for unitary transformations . . . . 97

A.2 Dispersive transformation . . . . . . . . . . . . 98

A.3 Rotating frame . . . . . . . . . . . . . 100

B Deriving the master equation 103

$\begin{array}{ll}\text { Bibliography } & 107\end{array}$

Appended papers $\quad 135$ 



\section{List of figures}

1.1 A micrograph of the artificial atom used to mediate photonphoton interactions in Paper III . . . . . . . . . . . . . 3

1.2 An artist's impression of an artificial atom coupled to SAWs 6

1.3 Sketch of an artificial atom probing quantum vacuum fluctuations in front of a mirror . . . . . . . . . . . 9

1.4 The Bloch sphere representation of a qubit . . . . . . 10

1.5 Quantum error-correction code for bit-flip protection with three qubits . . . . . . . . . . . . . 11

2.1 The three basic circuit elements for superconducting circuits 17

2.2 Circuit diagram for a transmission line . . . . . . . . 18

2.3 Circuit diagram for a Cooper-pair box . . . . . . . . 22

2.4 Energy levels of the Cooper-pair box . . . . . . . . . 23

2.5 SAW propagation . . . . . . . . . . . . 28

2.6 Sketch of an interdigital transducer . . . . . . . . . . . . 29

2.7 Circuit models for transmitter and receiver IDTs . . . . . . 31

2.8 Circuit model of a transmon coupled to SAWs . . . . . . . . 32

2.9 A model for a giant artificial atom . . . . . . . . . . . 34

2.10 A transmission line resonator coupled to two transmons . . 36

2.11 Bare and dressed states in the Jaynes-Cummings model . . 38

3.1 A Fock-state wavepacket interacting with a quantum system 51

3.2 Excitation probability for a two-level atom hit by a singlephoton wavepacket . . . . . . . . . . 55

4.1 Bloch sphere representation of two weakly entangled qubits 58

4.2 Illustration of the model for homodyne detection. . . . . . . 64

5.1 Cascading quantum systems . . . . . . . . . . 70 
5.2 The three operations in the $(S, L, H)$ formalism $\ldots \ldots \ldots 72$

5.3 Two coupled cavities . . . . . . . . . . . . . 74

$5.4(S, L, H)$ for a giant artificial atom $\ldots \ldots \ldots 78$

6.1 Cascaded transmons for photon detection $\ldots \ldots \ldots$. . . 87 


\title{
Nomenclature
}

\author{
Abbreviations \\ 1D, 2D, 3D One-dimensional, Two-dimensional, Three-dimensional \\ 2DEG Two-dimensional electron gas \\ CNOT Controlled-NOT (quantum computing gate) \\ CPB Cooper-pair box \\ CQED Cavity quantum electrodynamics \\ cQED Circuit quantum electrodynamics \\ GaAs Gallium arsenide \\ H.c. Hermitian conjugate \\ IDT Interdigital transducer \\ $\mathrm{LiNbO}_{3} \quad$ Lithium niobate \\ LO Local oscillator \\ QED Quantum electrodynamics \\ QND Quantum nondemolition \\ RWA Rotating wave approximation \\ SAW Surface acoustic wave \\ SME Stochastic master equation \\ SNR Signal-to-noise ratio \\ SQUID Superconducting quantum interference device
}

\section{Symbols defined in Chapter 1}

$\alpha, \beta \quad$ Complex numbers (amplitudes of a qubit state)

$\hbar \quad h / 2 \pi$

$\left|\psi_{3}\right\rangle,\left|\psi_{3, \text { err }}\right\rangle \quad$ A state with three qubits before/after a bit flip error

$|\psi\rangle \quad$ An arbitrary qubit state 


$\begin{array}{ll}\omega_{\mathrm{p}}, \omega_{\mathrm{c}} & \text { Probe frequency, control frequency } \\ \omega & \text { Angular frequency } \\ \theta, \phi & \text { Angles on the Bloch sphere } \\ a, a^{\dagger} & \text { Annihilation/creation operator for a harmonic oscillator } \\ c & \text { The speed of light } \\ H & \text { Hamiltonian } \\ h & \text { Planck's constant } \\ L & \text { Distance from an artificial atom to a mirror } \\ m & \text { Mass } \\ M_{12}, M_{23} & \text { Parity measurement (result) for qubits } 1 \text { and } 2(2 \text { and } 3) \\ N & \text { Number of qubits } \\ x, p & \text { Position and momentum of a harmonic oscillator }\end{array}$

\section{Symbols defined in Chapter 2}

$\chi$

$\Delta x$

$\Delta$

$\delta(x)$

$\delta_{n m}$

$\eta$

$\frac{\partial \mathfrak{L}}{\partial \dot{\Phi}_{n}}$

$\Gamma$

$|g / e\rangle$

$|n, \pm\rangle$

$|n\rangle$

$\lambda$

$\lambda$

a

D

E

$\mathbf{F}, F_{i}$

$\mathrm{S}$

$\mathbf{T}$

$\mathbf{u}, u_{i}$

$\mathcal{L}$
Dispersive frequency shift

A small distance

Detuning between atom and resonator frequencies, $\omega_{\mathrm{a}}-\omega_{\mathrm{r}}$

Delta function

Kronecker delta: $\delta_{n m}=1$ if $n=m, \delta_{n m}=0$ if $n \neq m$

IDT metallization ratio

Generalized momenta

Transmon relaxation rate

Bare ground/excited state of a two-level atom

Dressed state

Bare resonator state with $n$ photons

$g / \Delta$ (a small parameter)

Wavelength

Acceleration

Electrical displacement

Electric field

Force (in direction $i=x, y, z$ )

Strain vector

Stress vector

Particle displacement (in direction $i=x, y, z$ )

Lagrangian density 
$\mathcal{P}$

$\mathfrak{L}$

$\mathfrak{L}_{\mathrm{JJ}}$

$\mathfrak{L}_{\mathrm{TL}}$

$\mathfrak{L}_{C}, \mathfrak{L}_{L}$

$\mathfrak{L}_{\mathrm{CPB}}$

$\mu$

$\omega_{\mathrm{a}}, \omega_{\mathrm{tr}}$

$\omega_{\mathrm{r}}$

$\omega_{j}$

$\omega_{m}$

$\Omega_{n}$

$\omega_{n}$

$\phi_{\mathrm{L}}, \phi_{\mathrm{R}}$

$\Phi$

$\phi$

$\phi$

$\Phi(x, t)$

$\Phi_{\mathrm{L}}, \Phi_{\mathrm{R}}$

$\Phi_{n}$

$\rho_{m}$

$\sigma_{i}$

$\sigma_{-}, \sigma_{+}$

$\sigma_{-}^{m}$

$\sigma_{+}^{m}$

$\varepsilon$

$\varepsilon_{0}$

$\varepsilon_{\mathrm{p}}$

$\varphi_{n}$

$\zeta$

a

$A_{j}$

$a_{\mathrm{L}, \omega}, a_{\mathrm{R}, \omega}$
Principal value

Lagrangian

Lagrangian for a Josephson junction

Lagrangian for a transmission line

Lagrangian for a capacitor/inductor

CPB Lagrangian

Coupling between IDT voltage and the electric potential for generated SAWs

Atom/transmon frequency

Resonator frequency

Frequency of mode $j$ in a transmission line

Energy of level $m$ in a multi-level atom $(\hbar=1)$

Splitting between dressed states, $\Omega_{n}=\sqrt{\Delta^{2}+4 g^{2}(n+1)}$

Resonance frequency for mode $n$ of a harmonic oscillator

Electric potential of left/right-moving SAWs

Flux

Electric potential at the surface of a piezoelectric solid

Phase difference across a Josephson junction

Flux density

Left/right-moving flux wave

Flux at node $\mathrm{n}$

Mass density

The Pauli matrices $(i=x, y, z)$

Atom lowering and raising operators

Lowering operator for a multi-level atom, $|m\rangle\langle m+1|$

Raising operator for a multi-level atom, $|m+1\rangle\langle m|$

Permittivity matrix

Permittivity of the medium above a SAW substrate

Permittivity of a SAW substrate

Mixing angle of a dressed state $|n, \pm\rangle$

Damping factor of a parallel $R L C$ circuit

IDT finger width

Cross-section area in direction $j(j=x, y, z)$

Annihilation operator for left/right-moving photons of angular frequency $\omega$ 


\begin{tabular}{|c|c|}
\hline$a_{\mathrm{L} j}, a_{\mathrm{R} j}$ & $\begin{array}{l}\text { Annihilation operator for left/right-moving photons in mode } \\
j \text { of a transmission line }\end{array}$ \\
\hline$C$ & Capacitance \\
\hline$c$ & Stiffness matrix \\
\hline$c^{\prime}$ & Renormalized stiffness in a piezoelectric solid \\
\hline$C_{0}, L_{0}$ & Capacitance/inductance per unit length \\
\hline$C_{\mathrm{g}}, V_{\mathrm{g}}$ & Gate capacitance/voltage \\
\hline$C_{\mathrm{J}}$ & Capacitance of a Josephson junction \\
\hline$C_{\mathrm{T}}$ & IDT capacitance \\
\hline$c_{\mathrm{g}}$ & Geometry factor for an IDT \\
\hline$C_{\mathrm{s}}$ & $\varepsilon_{0}+\varepsilon_{\mathrm{p}}$ \\
\hline$C_{\mathrm{tr}}$ & Transmon capacitance \\
\hline$c_{i j k l}$ & Elasticity tensor \\
\hline$e$ & Piezoelectric constant \\
\hline$e$ & The elementary charge \\
\hline$E(n, \pm)$ & Energy of a dressed state \\
\hline$E_{\mathrm{J}}$ & Josephson energy \\
\hline$E_{\mathrm{C}}$ & Electron charging energy \\
\hline$f$ & Frequency \\
\hline$f_{0}$ & IDT resonance frequency, $f_{0}=\lambda v_{0}$ \\
\hline$g$ & Coupling strength between resonator and atom \\
\hline$G_{\mathrm{a}}, B_{\mathrm{a}}$ & Real/imaginary part of the acoustic admittance \\
\hline$g_{m}$ & $\begin{array}{l}\text { Coupling between electric potential of incoming SAWs and } \\
\text { generated IDT current }\end{array}$ \\
\hline$g_{j k m}$ & $\begin{array}{l}\text { Coupling between transition } m-1 \text { in a multi-level atom and } \\
\text { mode } j \text { in a transmission line at point } x_{k}\end{array}$ \\
\hline$H_{\text {atom }}$ & Atom Hamiltonian \\
\hline$H_{\text {int }}$ & Interaction Hamiltonian \\
\hline$H_{\mathrm{Rabi}}, H_{\mathrm{JC}}$ & Rabi Hamiltonian, Jaynes-Cummings Hamiltonian \\
\hline$H_{\mathrm{TL}}$ & Hamiltonian for a transmission line \\
\hline$H_{\mathrm{CPB}}$ & CPB Hamiltonian \\
\hline$I, I_{n}$ & Current (at node $n$ ) \\
\hline$I_{\mathrm{C}}$ & Critical current in a Josephson junction \\
\hline$I_{\mathrm{J}}$ & Supercurrent in a Josephson junction \\
\hline$k$ & Wavenumber, $\omega / v$ \\
\hline
\end{tabular}


$K^{2} \quad$ Electromechanical coupling in a piezoelectric solid

L Inductance

$L_{\mathrm{J}} \quad$ Josephson inductance

$N \quad$ Number of connection points between a giant artificial atom and a $1 \mathrm{D}$ transmission line

$n \quad$ Number of Cooper pairs on the CPB island

$n_{\mathrm{g}} \quad$ Background charge, measured in Cooper pairs

$N_{\mathrm{p}} \quad$ Number of IDT finger pairs

$P \quad$ Power

$p \quad$ IDT inter-finger distance

$Q(x, t) \quad$ Charge density

$Q_{n} \quad$ Charge at node $\mathrm{n}$

$R \quad$ Resistance

$r \quad$ Atom radius

$S_{i j} \quad$ Strain tensor, $\frac{\partial u_{i}}{\partial j}$

$S_{V V}[\omega] \quad$ Spectral density for voltage fluctuations at $\omega$

$T_{i j} \quad$ Stress tensor, $F_{i} / A_{j}$

$v \quad$ (Particle displacement) velocity

$V, V_{n} \quad$ Voltage (at node $n$ )

$v_{0} \quad$ SAW propagation velocity

$V_{\mathrm{T}} \quad$ Voltage source connected to an IDT

$v_{\text {wave }} \quad$ Acoustic wave velocity

$W \quad$ IDT finger length, width of a SAW beam

$x_{k} \quad$ Coordinate of IDT electrode $k$ or of coupling point $k$ for a giant multi-level atom interacting with a $1 \mathrm{D}$ transmission line

$Y_{0} \quad$ Transmission line conductance

$y_{0} \quad$ Characteristic conductance for SAWs

$Z_{0} \quad$ Characteristic impedance of a transmission line

$Z_{\mathrm{L}}, Z_{\mathrm{S}} \quad$ Load/source impedance

\section{Symbols defined in Chapter 3}

$\bar{n}\left(\omega_{j}, T\right) \quad$ Number of thermal photons in mode $j$ at temperature $T$

$\Delta \quad$ Detuning between atom and incoming photon, $\omega_{\mathrm{a}}-\omega_{\mathrm{ph}}$

$\Gamma \quad$ Atom relaxation rate

$\Gamma_{\mathrm{ph}} \quad$ Bandwidth of a Gaussian photon wavepacket 
$\left|1_{\xi}\right\rangle,\left|N_{\xi}\right\rangle \quad$ A propagating Fock state with $1 / N$ photons and envelope $\xi$

$\left|\psi_{0}\right\rangle$

$|\psi\rangle$

$\mathcal{D}[c] \rho$

$\mathbf{1}_{\text {bath }}$

$\mathcal{T}$

$\omega_{a}^{\prime}, \omega_{a}^{\prime \prime}$

$\omega_{\mathrm{ph}}$

$\mathrm{d} \Lambda(t)$

$\mathrm{d} B_{t}$

$\mathrm{d} U_{t}$

$\rho$

$\rho_{\text {atom }}, \rho_{\text {bath }}$

$\rho_{\text {tot }}$

$\rho_{m, n}$

$\tilde{\xi}(\omega)$

$\tilde{X}(t)$

$\xi(t)$

$b(\omega)$

$b_{0}(\omega), b_{1}(\omega)$

$b_{\text {in }}(t), b_{\text {out }}(t)$

$b_{j}$

$B_{t}$

$c$

$g_{j}$

$H_{\text {bath }}$

$J(\omega)$

$k_{\mathrm{B}}$

$L$

$P_{\text {exc }}(t)$

$T$

$t, \tau$

$t_{0}, t_{1}$

$t_{\mathrm{ph}}$

$U(t), U(t, 0)$
A pure atom state at time $t=0$

The state of a quantum system

Lindblad superoperator, $\mathcal{D}[c] \rho=c \rho c^{\dagger}-\frac{1}{2} c^{\dagger} c \rho-\frac{1}{2} \rho c^{\dagger} c$

The identity operator in the bath Hilbert space

The time-ordering operator

Renormalized atom frequency at negligible/finite $T$

Photon frequency

Gauge process increment

A quantum noise increment

A small increment of the time evolution operator

(Effective) density matrix

Atom/bath density matrix

Density matrix for the total system (atom plus bath)

Generalized density matrix for Fock-state input

Spectral density function of a propagating Fock state

An arbitrary operator $X$ in the interaction picture

The temporal shape of a propagating Fock state

Annihilation operator for photons of frequency $\omega$

$b\left(\omega, t=t_{0}\right), b\left(\omega, t=t_{1}\right)$ in the Heisenberg picture

$t)$ The in/out-field in input-output theory

Annihilation operator for mode $j$ in a bath

A quantum Wiener process

An arbitrary operator of a quantum system

Coupling between an atom and mode $j$ of the bath

Hamiltonian for a bath/environment

Density of states for the bath

Boltzmann's constant

Coupling operator with relaxation rate, $\sqrt{\Gamma} \sigma_{-}$or $\sqrt{\kappa} a$

Probability of finding an atom in the excited state

Temperature

Time

An initial/future time

Arrival time for the center of a Gaussian photon wavepacket

The time evolution operator (from time 0 to time $t$ ) 
$U_{t} \quad$ The time evolution operator (from time 0 to time $t$ )

$w_{i} \quad$ Probability weights in the definition of the density matrix

\section{Symbols defined in Chapter 4}

$\alpha(t)$

$\beta$

$\chi_{d c}$

$\Delta\langle d\rangle$

$\epsilon$

$\eta$

$\mathrm{E}[X]$

$\kappa$

$|\Psi\rangle_{\mathrm{s}+\mathrm{p}}$

$|\Psi\rangle_{\mathrm{s}}$

$\mathcal{L}$

$\mathcal{G}[c] \rho$

$\mathcal{M}[c] \rho$

$\Omega_{i}(t)$

$\phi$

$\mathrm{d} A_{t}$

$\mathrm{d} C_{t}^{(1)}, \mathrm{d} C_{t}^{(2)}$

$\mathrm{d} N(t)$

$\mathrm{d} W(t)$

$\rho_{0}, \rho_{1}$

$\rho_{i}^{\mathrm{S}}, \rho_{i}$

$d$

$H_{0}, H_{1}$

$H_{\text {sys }}$

$j(t)$

$N(t)$

$N_{1}(t), N_{2}(t)$

$p_{i}$

$Y, Z$
A coherent probe signal

Amplitude of a coherent drive

Susceptibility connecting change of $d$ to perturbation of $c$

Change in $d$ due to a perturbation

Angle quantifying degree of entanglement for $|\Psi\rangle_{\mathrm{s}+\mathrm{p}}$

Measurement efficiency

Expectation value

Relaxation rate of a harmonic oscillator

Total state of system plus probe

System (qubit) state

Liouvillian

SME superoperator, $\mathcal{G}[c] \rho=\frac{c \rho c^{\dagger}}{\left\langle c^{\dagger} c\right\rangle}-\rho$

SME superoperator, $\mathcal{M}[c] \rho=c \rho+\rho c^{\dagger}-\left\langle c+c^{\dagger}\right\rangle \rho$

Operator giving measurement result $i, \Omega_{i}(t)=\langle i|U(t)| 0\rangle$

Phase set by the LO in homodyne detection

A quantum noise increment

Quantum noise increments for beamsplitter outputs Stochastic increment of $N(t)$

A Wiener increment

Unperturbed density matrix and a small perturbation

System density matrix, given measurement result $i$

An arbitrary system operator

Unperturbed Hamiltonian and a small perturbation

System Hamiltonian

Homodyne current

Stochastic process, counting number of photons detected

Stochastic process for photon counting at detector $1 / 2$

Probability of measurement outcome $i$

Result of measurement in the $Y / Z$ basis of a qubit 


\section{Symbols defined in Chapter 5}

$\alpha$

$\boxplus$

$\Delta_{\mathrm{a}}$

$\Delta_{a}, \Delta_{b}$

$\langle a\rangle_{\mathrm{ss}}$

$\Gamma$

$\Gamma_{k}$

$\kappa_{1}, \kappa_{2}$

$\kappa_{3}, \kappa_{4}$

$\kappa_{a}$

$\omega_{\beta}$

$\omega_{a}, \omega_{b}$

$\phi$

$\tilde{S}, \tilde{L}, \tilde{H}$

$\triangleleft$

G

$G_{1}, G_{2}$

$G_{\alpha}, G_{\beta}$

$G_{\phi}$

$G_{\mathrm{BS}}$

$G_{\mathrm{L}, 1}, G_{\mathrm{L}, 2}$

$G_{\mathrm{L}}, G_{\mathrm{R}}$

$G_{\mathrm{R}, 1}, G_{\mathrm{R}, 2}$

$G_{\text {tot }}$

$G_{a}, G_{b}$

$G_{a 1}, G_{a 2}$
Amplitude of a coherent signal

Concatenation product in the $(S, L, H)$ formalism

Detuning between atom and probe, $\omega_{\mathrm{a}}-\omega_{\mathrm{p}}$

$\omega_{a}-\omega_{\beta}, \omega_{b}-\omega_{\beta}$

The expectation value of $a$ in the steady state

Atom relaxation rate through each connection point

Atom relaxation rate through connection point $k$

Photon loss rate through the left/right side of a cavity with annihilation operator $a$

Photon loss rate through the left/right side of a cavity with annihilation operator $b$

Relaxation rate through each of the two sides of a cavity with annihilation operator $a$

Frequency of the coherent input signal

Frequency of a cavity with annihilation operator $a / b$

A phase shift

Scattering matrix, coupling operator vector, and Hamiltonian resulting from a feedback operation

Series product in the $(S, L, H)$ formalism

A doublet $(L, H)$ describing an open quantum system

$(S, L, H)$ triplet for system $1 / 2$

$(S, L, H)$ triplet for a coherent signal of amplitude $\alpha / \beta$

$(S, L, H)$ triplet for a phase shift $\phi$

$(S, L, H)$ triplet for a beamsplitter

$(S, L, H)$ triplet for the part of a giant atom interacting with left-travelling modes via connection point $1 / 2$

$(S, L, H)$ triplet for the part of a giant atom interacting with left/right-travelling modes

$(S, L, H)$ triplet for the part of a giant atom interacting with right-travelling modes via connection point $1 / 2$

Total $(S, L, H)$ triplet for a giant atom

$(S, L, H)$ triplet for a cavity with annihilation operator $a / b$

$(S, L, H)$ triplet describing input-output port $1 / 2$ of a cavity with annihilation operator $a$ 
$G_{b \rightarrow c} \quad(S, L, H)$ triplet for a system where the output from port $b$ is used as input for port $c$

$G_{b 1}, G_{b 2} \quad(S, L, H)$ triplet describing input-output port $1 / 2$ of a cavity with annihilation operator $b$

$H_{1}, H_{2} \quad$ Hamiltonian of system $1 / 2$

$H_{a}, H_{b} \quad$ Hamiltonian for a cavity with annihilation operator $a / b$

$L_{1}, L_{2} \quad$ Coupling operator for systems $1 / 2$

$L_{n} \quad$ Coupling operator for input-output port $n$

$L_{[k]} \quad$ Coupling operator vector with row $k$ removed

$S \quad$ Scattering matrix

$S_{[k, l]} \quad$ Scattering matrix with row $k$ and column $l$ removed

$U_{\mathrm{d} t}^{(1)}, U_{\mathrm{d} t}^{(2)} \quad$ Time evolution for system $1 / 2$ during a short time $\mathrm{d} t$

$\mathbf{1}_{n} \quad$ The $n \times n$ identity matrix

\section{Symbols defined in Chapter 6}

$\chi_{\mathrm{K}} \quad$ Strength of Kerr interaction

$N \quad$ Number of transmons in the setup for Paper V

$t_{\text {off }} \quad$ Time when the coherent probe is turned off in Paper I

\section{Symbols defined in the appendices}

$|\tilde{\psi}\rangle$

$\lambda$

$\tilde{H}$

$b(t)$

G

$H_{0}, H_{\mathrm{I}}$

$S$

$s(t)$

$U$

$U_{\text {disp }}$

$U_{\text {rot }}$
A state transformed by $U$

A scalar

A Hamiltonian transformed by $U$

$\sum_{j} g_{j} b_{j} e^{-i \omega_{j} t}$

An operator

The non-interacting and interacting parts of $H_{\mathrm{JC}}$

The exponent in $U_{\text {disp }}$

$\sigma_{-} e^{-i \omega_{\mathrm{a}} t}$

A unitary transformation

The dispersive transformation

A transformation to a rotating frame 



\section{Chapter 1}

\section{Introduction}

Quantum optics is the study of interaction between light and matter at a fundamental level, where the physical description needs to include quantum mechanics to account for the dynamics of single photons and atoms. For a long time, it was not clear whether such a regime was accessible to experiments. In 1952, Erwin Schrödinger, one of the founding fathers of quantum mechanics, wrote "we are not experimenting with single particles, any more than we can raise Ichthyosauria in the zoo" [1]. At that time, there were experiments involving single particles, but the only experimental records were traces in cloud chambers and the like, i.e., the measurements were destructive.

Decades after Schrödinger's comment, experiments started to catch up with theory. The Nobel Prize in Physics 2012 was awarded to Serge Haroche and David Wineland for their contribution to this field over the years [2-4]. They had demonstrated that single atoms could be used to probe photon states in a microwave cavity [5-8] and, conversely, that single ions could be trapped, cooled, and probed with laser light [9-13]. In both cases, the measurements are gentle enough to allow for continuous manipulation of the fragile quantum systems. Incidentally, one of the first big achievements for both research groups was to create and measure a superposition state known as a "Schrödinger cat state" [14, 15].

While we now have the tools to test theoretical predictions from quantum optics in practice, the experiments are still hard to implement and suffer from some limitations. However, in recent years there has been tremendous progress in performing analogues of quantum optics experiments using other systems $[16,17]$. These systems, known as artificial 
atoms, can be designed to emulate relevant properties of natural atoms. One example is artificial atoms made of superconducting circuits, which can have a multi-level structure with transitions at microwave frequencies. The artificial atoms not only make it easier to investigate known aspects of quantum optics; they also open up exciting possibilities of exploring new regimes which are not found in natural atoms.

In this thesis, we pursue both paths. Firstly, we study systems where artificial atoms overcome experimental limitations for natural atoms, realizing clear demonstrations of various known quantum optics phenomena. Secondly, we show examples where artificial atoms take us to new quantum optics regimes not possible for natural atoms. Some of the work presented in the eight appended papers falls into both categories.

In the following part of the introduction, we survey developments and motivations driving the field of quantum optics with artificial atoms, such as the use of microwave circuitry and the quest for a quantum computer. We end with an overview of the thesis.

\subsection{Quantum optics in superconducting circuits}

As we have seen, quantum optics experiments were originally performed with natural atoms, sometimes placed in cavities formed by mirrors. This approach is known as cavity quantum electrodynamics (cavity QED or CQED) $[18,19]$. In the last few decades, other systems such as quantum dots [20], nitrogen-vacancy centers in diamond [21], and rare-earth ions in crystals [22] have also attracted attention. However, perhaps the most versatile and promising of the new experimental approaches to quantum optics is that of superconducting circuits [23-28], often referred to as circuit quantum electrodynamics (circuit QED or cQED).

In the case of superconducting circuits, transmission lines on a chip are used to guide microwave photons to and from artificial atoms. The artificial atoms come in different varieties, but they are all based on Josephson junctions $[29,30]$ in combination with traditional circuit elements like capacitances and inductances.

All the elements of the superconducting circuits can be manufactured on a chip with lithographic methods. This allows for detailed design of properties suitable for the experiments one has in mind. It is possible to set the transition frequencies of the artificial atoms as well as the coupling strength between the artificial atoms and the transmission lines (the envi- 


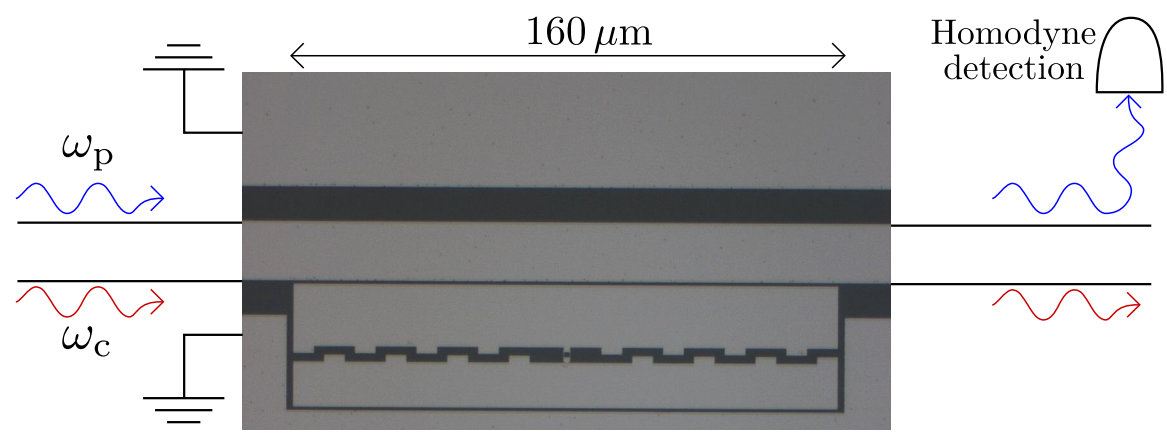

Figure 1.1: A micrograph of the artificial atom used to mediate photon-photon interactions in Paper III. The two sawtooth-shaped aluminium islands are coupled both capacitively and via Josephson junctions (the tiny structure connecting the islands in the middle). This superconducting circuit emulates a three-level atom, which couples to a transmission line for microwave photons (the middle aluminium strip with ground planes above and below). When the incoming probe photons at frequency $\omega_{\mathrm{p}}$ and control photons at $\omega_{\mathrm{c}}$ interact with the transitions of the artificial atom, the atom imparts a phase shift on the probe signal depending on the strength of the control signal. The phase-shifted probe signal is then read out using homodyne detection. Micrograph by Io-Chun Hoi.

ronment) with good precision; in fact, in some experiments one can even tune these important parameters in situ. The combination of easy-to-use conventional microwave electronics and a lithographic manufacturing process also means that there is good potential to scale up superconducting circuit setups to larger systems, which will be necessary in order to build a future quantum computer.

The control of superconducting circuits allows for some quantum optics experiments to be performed easier and more cleanly than is possible with natural atoms. This is the main reason why all experimental papers in this thesis (Papers III, IV, VI, and VIII) use superconducting circuits. The pure theory papers (Papers I, II, V, and VII) are also written with superconducting circuits in mind as the first experimental realization, but in most cases there are no insurmountable obstacles to implementing their suggested experiments in other systems.

A good example where superconducting circuits outperform natural atoms is provided in Paper III. In that paper, we use a three-level artificial atom in an open transmission line to mediate an effective photon-photon interaction between a probe signal and a control signal resonant with the first and the second atom transition, respectively, as shown in Fig. 1.1. We 
demonstrate phase shifts of tens of degrees in the probe signal when the control signal is on a single-photon level. Comparable experiments with natural atoms placed in optical fibres are at best able to achieve phase shifts of less than a milliradian per photon [31-33].

Another example is the experiment in Paper VIII. There, an artificial atom is placed close to the end of a transmission line. This setup mimics the situation of a natural atom placed in front of a mirror. While such an experiment has been performed with natural atoms [34], the superconducting circuit offers several advantages. One is that the artificial atom is fixed, but its effective distance to the mirror can be tuned by changing its transition frequency. The natural atom must be physically moved and is hard to keep rigidly in place. The other distinction between the two cases is that the superconducting circuit setup is effectively one-dimensional (1D), while the natural atom couples to a three-dimensional (3D) environment. These two differences make it easier to detect the interference effect of the mirror on the atom relaxation rate in the superconducting circuit. The move from $3 \mathrm{D}$ to $1 \mathrm{D}$ has in the last few years led to several experiments with artificial atoms in open transmission lines [35-41] (plus Papers III and VIII), significantly improving on earlier efforts with natural atoms [42-46] and quantum dots $[47,48]$, even though the latter ones use elaborate focussing to overcome the drawbacks of the $3 \mathrm{D}$ geometry.

From the discussion so far one might get the impression that superconducting circuits hold the answer to all problems in quantum optics. The reality is more complicated; superconducting circuits certainly have drawbacks compared to other systems. For example, two natural atoms of the same species are guaranteed to have identical features, but it is impossible to fabricate two artificial atoms with superconducting circuits and make sure that they are the same in every way. Another problem is the fact that the field of circuit QED is still young compared to other approaches, e.g., ion traps, and some tools available in experiments with optical photons are still missing from the toolkit for microwave superconducting circuits. An important example is an efficient single-photon detector for propagating photons, which exists in several variations for optical frequencies $[49,50]$, but is more difficult to achieve for microwave photons since their energies are several orders of magnitude lower than the energies of optical photons.

In Papers II and V, we try to remedy this drawback and investigate the limits of a possible photon-detection scheme in circuit QED (there are also other proposals [51-55]). The scheme is based on the Kerr-like photon- 
photon interaction we demonstrated experimentally in Paper III. A photon, with frequency close to that of the first transition in the artificial atom, is sent through a transmission line along with a coherent probe signal, which has a frequency close to that of the second transition of the artificial atom. If the atom can mediate a strong enough interaction between the probe and the photon, the presence of a photon can be read out from a measurement on the probe signal. While Paper II shows that it is not enough to use a single artificial atom to achieve sufficient signal-to-noise ratio (SNR) for photon detection, Paper $\mathrm{V}$ demonstrates that cascading several artificial atoms in the right way makes it possible. An important advantage compared to existing optical photon detectors is that ours does not destroy the photon to be detected; the detection is said to be quantum nondemolition (QND). Nondemolition detection schemes based on Kerr interactions for optical photons have also been suggested [56, 57], but seem harder to implement with natural atoms.

\subsection{Reaching new regimes with artificial atoms}

The advantages of superconducting circuits extolled in the previous section begs the question: if we can do things so much better with artificial atoms and have such freedom of design, can we not then reach new regimes inaccessible with natural atoms? Yes, we can! Comparing to the previous section, we here try to distinguish between parameter regimes that are merely very hard to reach with natural atoms (but are easier to achieve with artificial ones) and regimes which don't exist in nature, but can be designed for artificial atoms. The border between the two is not sharp.

Above, we focussed on quantum optics experiments with superconducting circuits and other artificial atoms. To fully appreciate the possibilities afforded by engineered quantum systems, we also need to introduce the field of quantum optomechanics, where the interaction between light and mechanical vibrations is studied [58-65]. The typical experimental setup is an optical cavity where one of the mirrors can move. The quantized vibrations of the mirror then couples to the photons in the cavity. This kind of setup has been realized in a large variety of systems in recent years [66-73]. Of special interest is quantum electromechanics, where the photons are provided in electrical circuits [74-78]. A corresponding experimental setup is then an $L C$-oscillator where one of the capacitor plates can vibrate, realizing a coupling to the microwave photons in the electrical 


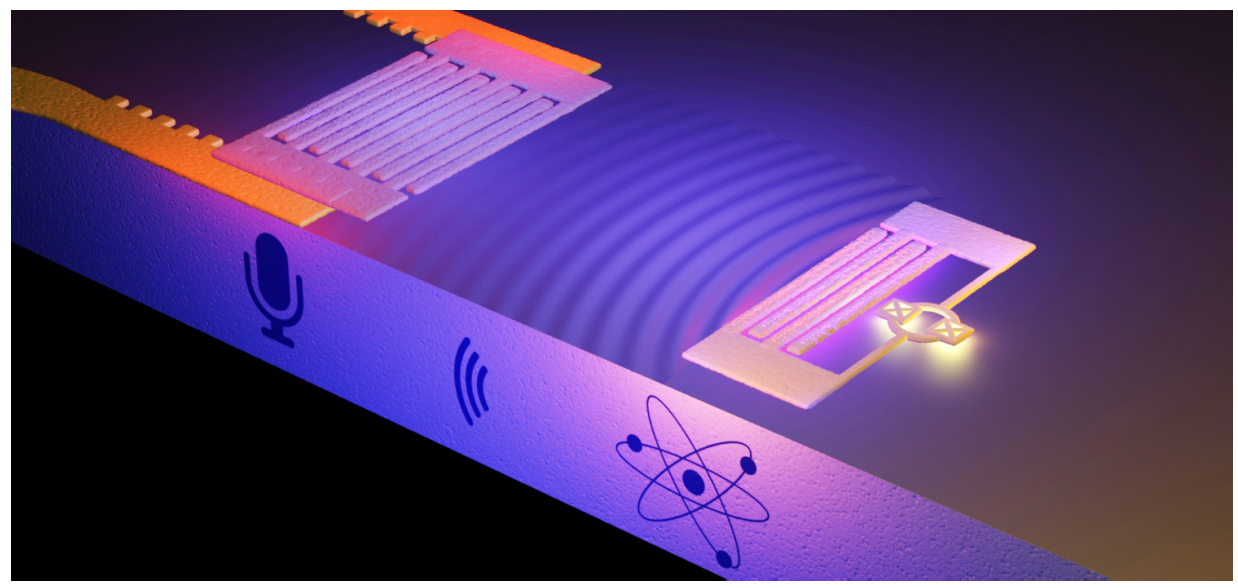

Figure 1.2: An artist's impression of an artificial atom coupled to SAWs. An interdigitated structure of metal fingers (an interdigital transducer, IDT) can convert microwave photons to phonons and vice versa. The structure to the left functions as both a loudspeaker and a microphone, letting us communicate with the artificial atom to the right via sound waves that travel on the surface of a microchip. Illustration by Philip Krantz (krantznanoart.com).

circuit. Mechanical vibrations have been cooled to their quantum ground state in both optomechanical and electromechanical setups in the last few years $[79,80]$. To complete the circle, there is now a theoretical proposal for implementing an analogue of optomechanics in superconducting microwave circuits without any moving parts [81]. Instead, two Josephson junctions form a loop to make a superconducting quantum interference device (SQUID) [30, 82], which can act as an effective movable mirror. The "movement" is achieved by tuning the magnetic field passing through the loop.

In the large zoo of artificial and natural atoms together with other engineered quantum systems, much of the interesting physics is a result of cross-breeding. There are many ongoing efforts to create hybrid systems that combine the best characteristics of different systems while avoiding their shortcomings [17, 83-92]. The general trend seems to be that superconducting circuits act as a hub for most of these efforts thanks to the ease with which such systems can be designed, manufactured, and controlled.

Papers VI and VII provide an excellent example of a hybrid system that uses mechanical vibrations and an artificial atom made from superconducting circuits to open up a new regime. In the experiment of Paper 
VI, illustrated in Fig. 1.2, surface acoustic waves (SAWs) are coupled to an artificial atom. The SAWs are vibrations that travel on the surface of a solid; they have seen widespread use in microwave technology, e.g., providing minute bandpass filters in cellphones [93-96]. Here, the SAWs propagate on a piezoelectric material, which makes it possible for them to contact the superconducting electrical circuit that is the artificial atom. For the first time, it is now possible to perform quantum optics experiments with sound taking on the role of light.

Since the sound travels much slower than light (a difference of roughly five orders of magnitude), but at the same frequency, it has a correspondingly shorter wavelength. This is what makes the experiment realize an entirely new regime, that of a "giant atom". In previous quantum optics studies with both natural and artificial atoms, the size of the atom was almost always negligible compared to the wavelength of the light. In the SAW setup, the atom is no longer point-like in this sense, but instead couples to the sound waves at several different points that can be wavelengths apart. This gives rise to new interference effects, resulting in frequency-dependent relaxation rates and energy level renormalizations for the artificial atom, which is the topic of the theoretical study in Paper VII.

Another area where superconducting circuits allow us improve experiments or reach new regimes is relativistic physics [97]. The SQUID emulation of a mirror can be made to "move" at an appreciable fraction of $c$, the speed of light. This has been used to demonstrate the dynamical Casimir effect [98-101], where a moving mirror creates pairs of photons from vacuum. There are also proposals to test the twin paradox on a chip [102] and the effect of relativity on quantum teleportation [103].

\subsection{Quantum fluctuations of the vacuum}

The relativistic physics that now is within reach for superconducting circuits is often connected to quantum vacuum fluctuations. These are at the heart of many quantum physics phenomena, including several that are relevant for the appended papers. As a basic example, consider the Hamiltonian for a harmonic oscillator,

$$
H=\frac{p^{2}}{2 m}+\frac{m \omega^{2} x^{2}}{2},
$$

where $p$ is the momentum, $m$ is the mass, $\omega$ is the angular frequency, and $x$ is the position of the oscillator. In quantum physics, $x$ and $p$ are operators 
with the commutation relation $[x, p]=i \hbar$, where $\hbar=h / 2 \pi$ ( $h$ is Planck's constant). Using this, and defining the annihilation and creation operators

$$
\begin{aligned}
a & =\sqrt{\frac{m \omega}{2 \hbar}}\left(x+\frac{i p}{m \omega}\right), \\
a^{\dagger} & =\sqrt{\frac{m \omega}{2 \hbar}}\left(x-\frac{i p}{m \omega}\right),
\end{aligned}
$$

the Hamiltonian can be rewritten as [104]

$$
H=\hbar \omega\left(a^{\dagger} a+\frac{1}{2}\right) .
$$

The operator $a^{\dagger} a$ counts the number of excitations of the oscillator. We see that even if the oscillator is in its ground state (zero excitations), it still has an energy $\frac{\hbar \omega}{2}$. This is the vacuum energy; the oscillator is never completely still.

The electromagnetic field can be described as a collection of harmonic oscillators where the excitations are photons $[105,106]$. Thus, in the electromagnetic vacuum there are photons flitting in and out of existence, which leads to several interesting effects. One example is the static Casimir effect [107], where two stationary mirrors in vacuum experience an attractive force due to there being fewer allowed electromagnetic field modes between them than elsewhere. The Casimir force is a result of more vacuum fluctuations pushing from the outside than from the inside. This effect has been detected experimentally [108]. Vacuum effects in relativistic settings include the dynamical Casimir effect mentioned above as well as Hawking radiation [109] and the Unruh effect [110].

The presence of vacuum fluctuations also affects atoms. A well-known example is the Lamb shift [111, 112], a renormalization of energy levels in the hydrogen atom. This kind of shift has also been measured for artificial atoms in superconducting circuits [113] and we calculate it for a giant artificial atom in Paper VII. Another effect of the vacuum fluctuations is that they induce relaxation of excited atom states [105, 114-116]. This is the quantum version of the fluctuation-dissipation theorem, which connects random fluctuations in the environment of a system with dissipation from that system [117-119]. Dissipation from a quantum system to an environment occurs in all the appended papers.

In Paper VIII, we use an artificial atom to map out the structure of vacuum fluctuations in front of a mirror (the end of a transmission line), as 


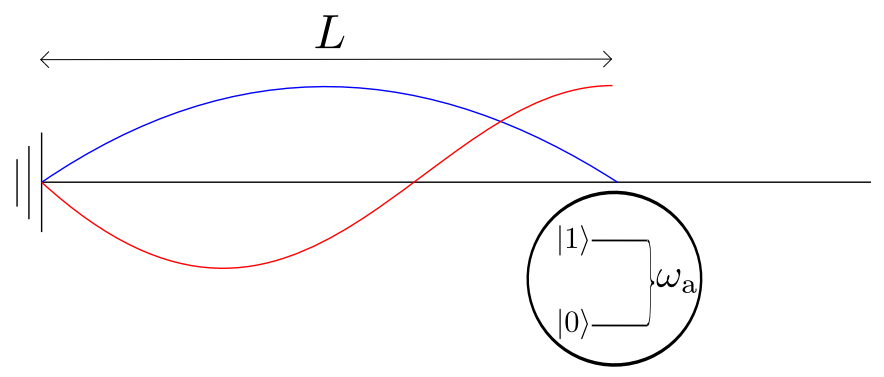

Figure 1.3: Sketch of an artificial atom probing quantum vacuum fluctuations in front of a mirror. The superconducting circuit that is the artificial atom is placed at a distance $L$ from the termination of a transmission line to ground, which is an effective mirror. By modulating the transition frequency $\omega_{\mathrm{a}}$ of the atom, the distance to the mirror can be changed in terms of wavelengths $\lambda=2 \pi c / \omega_{\mathrm{a}}$, where $c$ is the speed of light in the transmission line. Changing the effective distance $L / \lambda$ places the atom at a node (blue line) or antinode (red line) of vacuum fluctuation modes.

illustrated in Fig. 1.3. The information is extracted by measuring the relaxation rate of the atom as we tune its frequency, thus effectively changing its distance to the mirror.

Finally, quantum fluctuations of the vacuum are also important in the context of measurements on quantum systems [120]. The fluctuations result in an unavoidable noise background, which must be overcome. This is a vital point for the different measurement schemes analyzed in Papers I, II, and V.

\subsection{Quantum computing and parity measurement}

We have already alluded to the building of a quantum computer as an important motivation for much of the development in quantum optics in the last decades. The idea of a quantum computer was introduced by Feynman in 1982 [121]. In contrast to a classical computer, which operates using bits that can be in the two states 0 and 1 , a quantum computer would work with quantum bits, qubits. Qubits have eigenstates denoted $|0\rangle$ and $|1\rangle$, but they can exist in a superposition of these states,

$$
|\psi\rangle=\alpha|0\rangle+\beta|1\rangle
$$

where $\alpha$ and $\beta$ are complex numbers satisfying the normalization condition $|\alpha|^{2}+|\beta|^{2}=1[122]$. Such a superposition state can be represented on a 


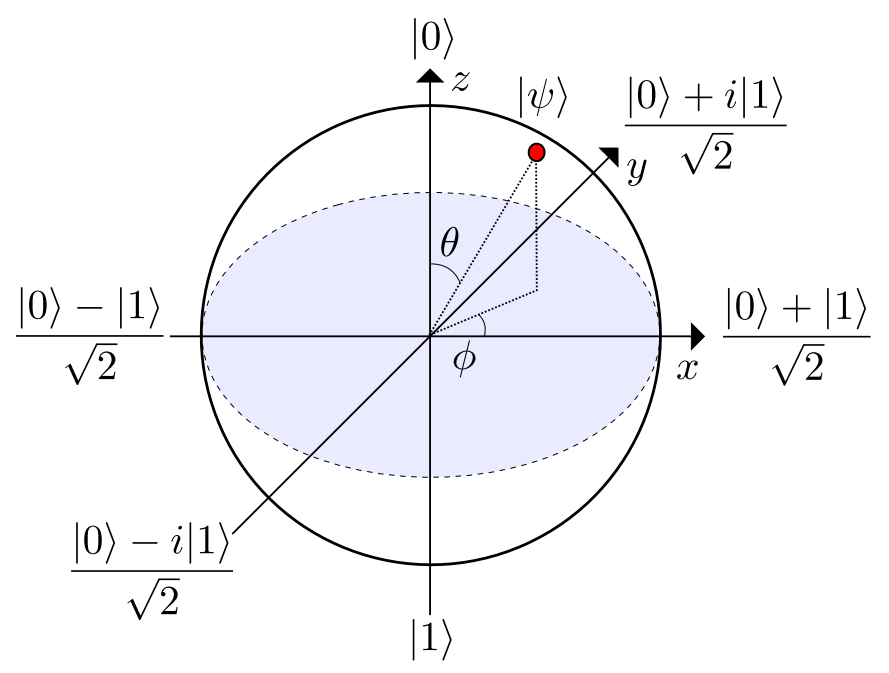

Figure 1.4: The Bloch sphere representation of a qubit. The basis states are located at the north and south poles. The various possible superpositions of the two can then be converted to unique coordinates on the sphere, since an equivalent parametrization of the superposition is $|\psi\rangle=\cos \frac{\theta}{2}|0\rangle+e^{i \phi} \sin \frac{\theta}{2}|1\rangle$.

Bloch sphere, shown in Fig. 1.4.

Simply put, the possibility of putting a qubit in superposition states enables a parallelization of computation that provides an advantage compared to classical computers. Several quantum computing algorithms have been developed that can provide a great speed-up for solving certain classes of problems [123-127].

To build a fully working quantum computer, an architecture must be found that is scalable and uses long-lived qubits that can both be measured and work in gate operations [128]. Several systems, with both natural and artificial atoms, are being investigated for this purpose [16, 26, 28, 129133]. However, so far only a few qubits have been made to work together [134-137] (not counting the D-Wave machines [138-144]). Hence, before we see large-scale quantum computers we will first have quantum simulators [145-152], where a smaller number of qubits are used to investigate quantum physics problems that are intractable on classical computers; it takes $2^{N}$ classical bits to simulate $N$ quantum two-level systems, but it only takes $N$ qubits.

It is also hard to make qubits that can maintain a superposition state for a long time. Coupling to environmental noise like vacuum fluctuations 


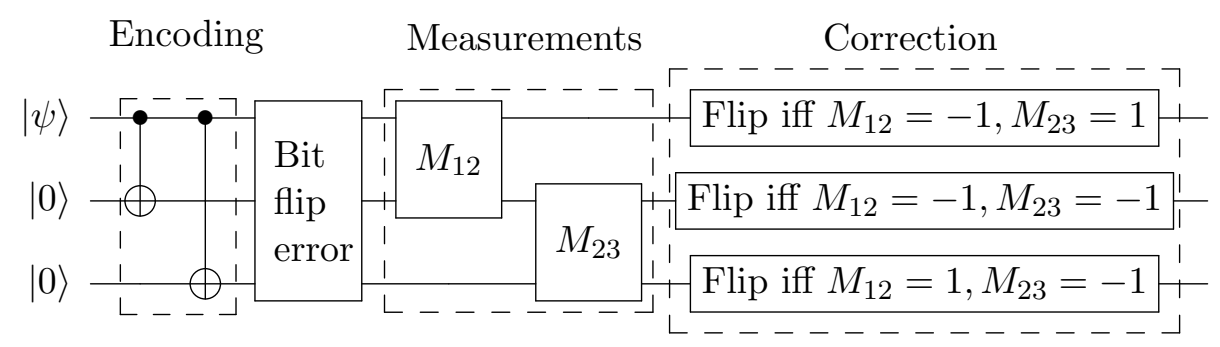

Figure 1.5: A circuit diagram for the three-qubit bit-flip error-correction code. In the first step, quantum controlled-NOT (CNOT) gates are applied to produce the state $\left|\psi_{3}\right\rangle$ from $|\psi\rangle$ (see Eq. (1.6)). After a bit-flip error occurs, parity measurements are done and correcting flips are applied to the qubits depending on the measurement results ( -1 means the qubits are in opposite states, +1 that they are in the same state).

leads to decoherence of the qubit. For superconducting qubits there has been tremendous progress in the last few years, increasing coherence times from microseconds to above a millisecond [153-155]. This is not enough in itself to enable computations with low enough error rate, but it is at the threshold for being useful in quantum error correction codes, where several qubits together represent and store the information of a single "logical qubit" [156-159]. This redundancy allows for a kind of "majority vote" system where if one qubit fails, that can be detected and corrected using the others.

In the last years, 2D surface codes have emerged as a good, scalable candidate for error correction in quantum computing [160,161]. These and other codes use parity measurements to detect errors without disturbing the encoded logical qubit. A parity measurement on two or more qubits is a measurement which determines whether an even or odd number of them are in the same state. The measurement does not give any information about the states of the individual qubits, preserving their superposition states. Thus a parity measurement on two qubits tells us if they are in either some superposition of $|00\rangle$ and $|11\rangle$ or in some superposition of $|01\rangle$ and $|10\rangle$. It does not give us any clue about whether any single qubit is in state $|0\rangle$ or $|1\rangle$.

The simple three-qubit code, which can protect against bit-flip errors, is a pedagogical example showing how parity measurements can be used in error correction [122] (and has been implemented with superconducting circuits [162]). The process is shown schematically in Fig. 1.5. We take 
our logical state $|\psi\rangle$ from Eq. (1.5) and encode it using three qubits as

$$
\left|\psi_{3}\right\rangle=\alpha|000\rangle+\beta|111\rangle .
$$

It is important to note that the quantum no-cloning theorem [163, 164] prevents us from merely creating three independent identical copies of $|\psi\rangle$; we have to make this entangled state instead. Now, let us assume that the third qubit is flipped. This gives

$$
\left|\psi_{3, \mathrm{err}}\right\rangle=\alpha|001\rangle+\beta|110\rangle .
$$

If we first do a parity measurement on qubits 1 and 2, and then on qubits 2 and 3 , we do not affect the state $\left|\psi_{3 \text {,err }}\right\rangle$. However, the result of the measurements lets us draw the conclusion that qubit 3 has been flipped (assuming that the probability of more than one qubit flipping is negligible). We can then apply a control pulse to this qubit, flipping it back to its original state.

In Paper I, we show how parity measurements in circuit QED can be improved by undoing unwanted measurement back-action. The setup we investigate has two qubits coupled to a resonator. By driving the resonator with a coherent microwave signal, and detecting the output in a suitable way, one can for certain system parameters realize a parity measurement of the two qubits [165]. However, the measurement also seems to give extra back-action on one of the parity states, which would make it unsuitable for practical use. In our paper, we are able to show that a careful analysis of the measurement signal reveals all the information about this extra backaction needed to undo it.

\subsection{Overview of the thesis}

This is a compilation thesis, consisting of an introductory text and appended reprints of eight papers. In the present chapter, we have given an overview of the field, placing the work of the appended papers in their proper context and explaining the motivation for them. In the next few chapters, we will mainly review the theoretical tools used in the appended papers. Although some of the appended papers include experiments, this is a theory thesis, and we defer to the appended papers in question, together with the theses of some of our experimental collaborators [40, 166-168], for details about fabrication and measurement setups. 
Chapter 2 is devoted to the various components of the systems we consider in the appended papers: transmission lines, surface acoustic waves, and artificial atoms in the form of superconducting qubits. We review how to formulate a quantum mechanical description of such electrical circuits and say a few words about the Jaynes-Cummings model, which describes interaction between an atom and a resonator.

Chapters 3-5 form the theoretical backbone of the thesis. In Chapter 3 , we begin to look at open quantum systems, where a small quantum system is coupled to an environment with a large number of degrees of freedom. To handle this situation, master equations are introduced which constitute an effective description of the system under the influence of the environment. This is used to some extent in all appended papers. From the master equation we then move on to input-output theory, which deals in more detail with excitations arriving at and leaving the small system.

The output from a system can be measured in various ways; this is the topic of Chapter 4. It is especially important for Papers I, II, and V, which deal with both parity measurements and photon detection. In these papers, as well as in Papers VII and VIII, we also make use of the $(S, L, H)$ formalism for cascaded quantum systems, which is the topic of Chapter 5 . Here, input-output theory is extended to handle output from one system being used as input for another.

Chapter 6 is an overview of the results in the appended papers. Briefly, Paper I analyzes a scheme for parity measurement in circuit QED and shows that an unwanted type of measurement back-action actually can be avoided by fully using the information in the measurement record. Papers II and V are theoretical investigations of a possible photon detector setup for circuit QED, where artificial atoms mediate an interaction between the photon to be detected and a coherent probe signal. In Paper II, we show that one atom is not enough to overcome the quantum background noise, but Paper $\mathrm{V}$ shows that several atoms cascaded in the right way can do it. Paper III is a proof-of-principle experiment demonstrating that an artificial atom in the form of a superconducting circuit can indeed mediate the strong photon-photon interactions we rely on in Papers II and V.

In Paper IV, we explain experimental results for an artificial atom coupled to photons in a resonator. The system exhibits rich dynamics when driven and probed with signals at different frequencies. Finally, Papers VI, VII, and VIII are all concerned with an artificial atom coupled to a bosonic field at several points, which can be wavelengths apart. In 
Paper VIII, an artificial atom placed in front of a mirror is used as a probe of the interference pattern in the mode structure of the quantum vacuum fluctuations. Paper VI is a ground-breaking experimental demonstration of coupling between an artificial atom and propagating sound in the form of SAWs. The short wavelength of the SAWs makes the atom "giant" in comparison; the effects of this new regime is explored further theoretically in Paper VII, which shows how the multiple coupling points of the atom give rise to interference effects affecting both the atom's relaxation rate and its energy levels.

Finally, we conclude in Chapter 7 by summarizing our work and looking to the future. There are indeed many interesting directions to pursue in the field of quantum optics with artificial atoms. 


\section{Chapter 2}

\section{Artificial atoms and 1D waveguides}

As we saw in Chapter 1, there are many systems that can be used for experiments in quantum optics. The archetypical setup is either single atoms or ions, trapped in an electromagnetic field, being manipulated with laser light $[2,4,11-13,15,129,135,136,148,169,170]$, or the reverse, light trapped between two mirrors interacting with passing atoms $[2,3,5-8,18$, $19,171,172]$. However, in the last decade or so, an increasing number of quantum optics experiments have been done using artificial atoms in superconducting circuits $[23-28,35,37,41,101,113,137,162]$. The versatility offered by superconducting circuits in designing the artificial atoms and their couplings to the surroundings, as well as the simplicity of using existing microwave technology for the signal processing in experiments, are the main reasons behind this development.

Since all experimental papers in this thesis use superconducting circuits, and all the theoretical papers are written mainly with such implementations in mind, this chapter is devoted to the quantum theory of electrical circuits. After we introduce the theoretical tools needed to go from a classical circuit description to a quantum one, we will illustrate their use on our two main components: the transmission line and the artificial atom known as a transmon [173]. We will also look at theory for surface acoustic waves in piezoelectric materials and show how a transmon can couple to such waves. Finally, we say a few words about regimes in the Jaynes-Cummings model [174], which describes interactions between an atom and photons in a resonator. 


\subsection{Circuit QED}

The process for quantizing circuits has already been well described in Refs. [175-179]. In the following, we will cover the main points in these references.

To arrive at a quantum description given an electrical circuit, the first step is to write down the classical Lagrangian $\mathfrak{L}$ [180] of the circuit. It turns out to be convenient to work with node fluxes

$$
\Phi_{n}(t)=\int_{-\infty}^{t} V_{n}\left(t^{\prime}\right) \mathrm{d} t^{\prime},
$$

where $V_{n}$ denotes node voltage at node $n$, and node charges

$$
Q_{n}(t)=\int_{-\infty}^{t} I_{n}\left(t^{\prime}\right) \mathrm{d} t^{\prime}
$$

where $I_{n}$ denotes node current. With the node fluxes as our generalized coordinates, the Hamiltonian $H$ follows from the Legendre transformation [180]

$$
H=\sum_{n} \frac{\partial \mathfrak{L}}{\partial \dot{\Phi}_{n}} \dot{\Phi}_{n}-\mathfrak{L} .
$$

The generalized momenta $\frac{\partial \mathfrak{L}}{\partial \dot{\Phi}_{n}}$ will sometimes, but not always, be the node charges $Q_{n}$.

Up to this point, everything has been completely classical. To proceed to quantum mechanics, we promote the generalized coordinates and momenta to operators with the canonical commutation relation

$$
\left[\Phi_{n}, \frac{\partial \mathfrak{L}}{\partial \dot{\Phi}_{m}}\right]=i \hbar \delta_{n m}
$$

where $\delta_{n m}$ is the Kronecker delta.

For quantum optics with superconducting circuits, three basic elements are needed: capacitors, inductors, and Josephson junctions, illustrated in Fig. 2.1. A Josephson junction consists of a thin insulating barrier between two superconducting leads, and it can be modelled as a capacitor in parallel with a nonlinear inductor characterized by the Josephson energy $E_{\mathrm{J}}$.

The Lagrangians for capacitors and inductors are straightforward. The energy of a capacitor with capacitance $C$ is

$$
\frac{C V^{2}}{2}=\frac{C\left(\dot{\Phi}_{1}-\dot{\Phi}_{2}\right)^{2}}{2},
$$



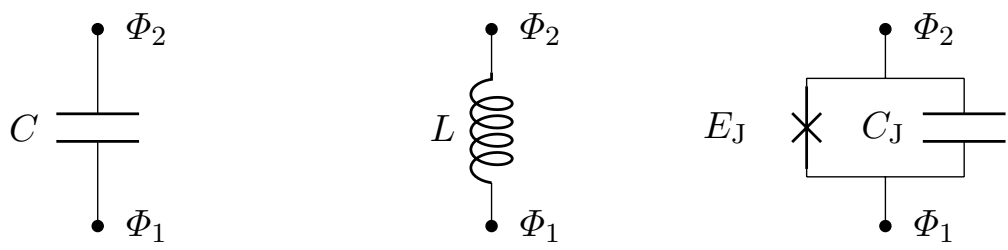

Figure 2.1: The three basic circuit elements used in quantum optics for superconducting circuits. From left to right: capacitance $C$, inductance $L$, and a Josephson junction with capacitance $C_{\mathrm{J}}$ and Josephson energy $E_{\mathrm{J}}$.

where $V$ is the voltage across the capacitor, and for an inductor with inductance $L$ it is

$$
\frac{L I^{2}}{2}=\{V=L \dot{I}\}=\frac{\left(\Phi_{1}-\Phi_{2}\right)^{2}}{2 L},
$$

where $I$ is the current through the inductor. In the Lagrangian, capacitive terms (terms with $\dot{\Phi}$ ) represent kinetic energy and give a positive contribution, while inductive terms (terms with $\Phi$ ) represent potential energy and give a negative contribution. We thus have

$$
\begin{aligned}
\mathfrak{L}_{C} & =\frac{C\left(\dot{\Phi}_{1}-\dot{\Phi}_{2}\right)^{2}}{2}, \\
\mathfrak{L}_{L} & =-\frac{\left(\Phi_{1}-\Phi_{2}\right)^{2}}{2 L} .
\end{aligned}
$$

For the Josephson junction, the contribution from the capacitive part with $C_{\mathrm{J}}$ follows immediately from previous discussion. To get the contribution from the nonlinear inductor, we use the Josephson equations [29, $30]$

$$
\begin{aligned}
I_{\mathrm{J}} & =I_{\mathrm{C}} \sin \phi, \\
\dot{\phi} & =\frac{2 e}{\hbar} V(t),
\end{aligned}
$$

where $I_{\mathrm{J}}$ is the supercurrent through the junction, $I_{\mathrm{C}}$ is the critical current (the maximum value of $\left.I_{\mathrm{J}}\right), V(t)$ is the voltage across the junction, $\phi=2 e\left(\Phi_{1}-\Phi_{2}\right) / \hbar$ is a phase difference across the junction, and $e$ is the elementary charge. These equations give

$$
\int_{-\infty}^{t} I\left(t^{\prime}\right) V\left(t^{\prime}\right) \mathrm{d} t^{\prime}=E_{\mathrm{J}}(1-\cos \phi)
$$




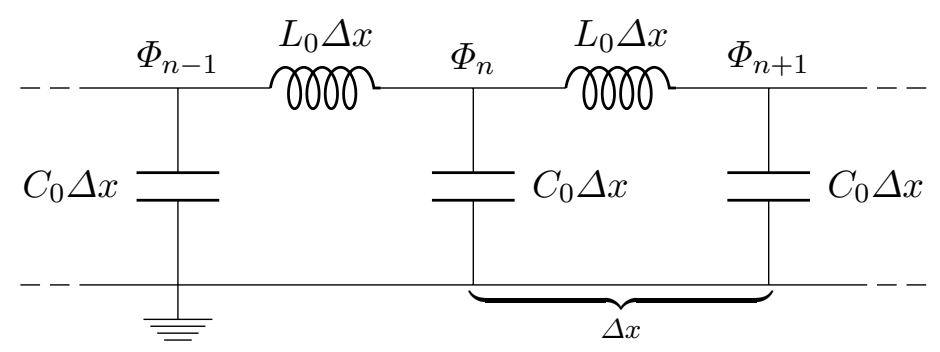

Figure 2.2: Circuit diagram for a transmission line. $C_{0}$ and $L_{0}$ denote capacitance per unit length and inductance per unit length, respectively, and $\Delta x$ is a small distance which will go to zero in the continuum limit.

where we have identified the Josephson energy $E_{\mathrm{J}}=\hbar I_{\mathrm{C}} / 2 e$. The Lagrangian for the Josephson junction is thus

$$
\mathfrak{L}_{\mathrm{JJ}}=\frac{C_{\mathrm{J}}\left(\dot{\Phi}_{1}-\dot{\Phi}_{2}\right)^{2}}{2}-E_{\mathrm{J}}(1-\cos \phi) .
$$

Note that the inductive term is a cosine function rather than the quadratic function for a normal inductor; this is why the Josephson junction can be seen as nonlinear inductance. This nonlinearity is essential for making artificial atoms with different level structures. From normal capacitors and inductors we can only get harmonic $L C$-oscillators.

\subsection{The quantized transmission line}

With the formalism for quantum circuits in hand, we now apply it to our first building block in quantum optics with superconducting circuits: the transmission line. A microwave transmission line is basically a coaxial cable squashed onto a chip; it consists of a center conductor between two ground planes. We will first consider an infinitely long transmission line and then insert mirrors (gaps) to form resonators.

\subsubsection{The infinite 1D waveguide}

The transmission line can be modelled by the circuit depicted in Fig. 2.2 [181, 182]. Using Eqs. (2.7) and (2.8), we immediately get the Lagrangian

$$
\mathfrak{L}_{\mathrm{TL}}=\sum_{n}\left[\frac{C_{0} \Delta x}{2}\left(\dot{\Phi}_{n}(t)\right)^{2}-\frac{1}{2 L_{0} \Delta x}\left(\Phi_{n+1}(t)-\Phi_{n}(t)\right)^{2}\right],
$$


from which we can identify the conjugate momenta

$$
\frac{\partial \mathfrak{L}_{\mathrm{TL}}}{\partial \dot{\Phi}_{n}}=C_{0} \Delta x \dot{\Phi}_{n}(t)
$$

which are the node charges $Q_{n}(t)$. Applying the Legendre transformation (Eq. (2.3)) to $\mathfrak{L}_{\mathrm{TL}}$, inserting the definition of the node charges, and taking the limit $\Delta x \rightarrow 0$ (or rather $\Delta x \rightarrow \mathrm{d} x$ ) gives the Hamiltonian

$$
H_{\mathrm{TL}}=\frac{1}{2} \int_{-\infty}^{\infty} \mathrm{d} x\left(\frac{Q(x, t)^{2}}{C_{0}}+\frac{1}{L_{0}}\left(\frac{\partial \Phi(x, t)}{\partial x}\right)^{2}\right),
$$

where $Q(x, t)$ and $\Phi(x, t)$ are charge density and flux density, respectively. The Lagrangian can also be written in a continuum form

$$
\mathfrak{L}_{\mathrm{TL}}=\int_{-\infty}^{\infty} \mathrm{d} x \mathcal{L}=\int_{-\infty}^{\infty} \mathrm{d} x\left(\frac{C_{0}}{2}(\dot{\Phi}(x, t))^{2}-\frac{1}{2 L_{0}}\left(\frac{\partial \Phi(x, t)}{\partial x}\right)^{2}\right),
$$

and applying the Euler-Lagrange equations [106]

$$
\frac{\partial}{\partial \mu}\left(\frac{\partial \mathcal{L}}{\partial\left(\frac{\partial \Phi}{\partial \mu}\right)}\right)-\frac{\partial \mathcal{L}}{\partial \Phi}=0, \quad \mu=x, t
$$

to the Lagrangian density $\mathcal{L}$ gives the wave equation

$$
\frac{\partial^{2} \Phi(x, t)}{\partial t^{2}}-\frac{1}{L_{0} C_{0}} \frac{\partial^{2} \Phi(x, t)}{\partial x^{2}}=0 .
$$

This tells us that there will be left- and right-moving flux waves

$$
\Phi(x, t)=\Phi_{\mathrm{L}}(k x+\omega t)+\Phi_{\mathrm{R}}(-k x+\omega t)
$$

moving in the transmission line with velocity $v=1 / \sqrt{L_{0} C_{0}}$ and wavenumber $k=\omega / v$.

So far, all calculations have been classical. To quantize the field in the transmission line, we promote the generalized coordinates and momenta to operators with the commutation relation

$$
\left[\Phi(x), Q\left(x^{\prime}\right)\right]=i \hbar \delta\left(x-x^{\prime}\right),
$$

where the delta function, rather than the Kronecker delta, appears since we are working with a continuum model. From the form of the Hamiltonian in 
Eq. (2.15) it can be seen that we have a collection of harmonic oscillators. We can thus rewrite the generalized coordinates and momenta in terms of annihilation and creation operators, just like in Sec. 1.3. The left- and right-moving fluxes become [175, 178, 183]

$$
\Phi_{\mathrm{L} / \mathrm{R}}(x, t)=\sqrt{\frac{\hbar Z_{0}}{4 \pi}} \int_{0}^{\infty} \frac{\mathrm{d} \omega}{\sqrt{\omega}}\left(a_{\mathrm{L} / \mathrm{R}, \omega} e^{-i( \pm k x+\omega t)}+\text { H.c. }\right),
$$

where H.c. denotes Hermitian conjugate, the annihilation and creation operators obey the commutation relations

$$
\left[a_{\mathrm{X}, \omega}, a_{\mathrm{X}^{\prime}, \omega^{\prime}}^{\dagger}\right]=\delta\left(\omega-\omega^{\prime}\right) \delta_{X X^{\prime}}
$$

and $Z_{0}=\sqrt{L_{0} / C_{0}}$ is the characteristic impedance of the transmission line.

To connect to the discussion of quantum vacuum fluctuations in Sec. 1.3 and the measurement of their strength in a semi-infinite transmission line in Paper VIII, it is illustrative to calculate the spectral density of the voltage fluctuations in our open transmission line using Eq. (2.21). Using $V=\partial_{t} \Phi$, we have $[120]$

$$
\begin{aligned}
S_{V V}[\omega]= & \int_{-\infty}^{\infty} \mathrm{d} t e^{i \omega t}\langle V(t) V(0)\rangle \\
= & \int_{-\infty}^{\infty} \mathrm{d} t e^{i \omega t} \frac{\hbar Z_{0}}{4 \pi} \int_{0}^{\infty} \frac{\mathrm{d} \omega^{\prime}}{\sqrt{\omega^{\prime}}} \int_{0}^{\infty} \frac{\mathrm{d} \omega^{\prime \prime}}{\sqrt{\omega^{\prime \prime}}}\left(-i \omega^{\prime}\right)\left(-i \omega^{\prime \prime}\right) \\
& \times\left\langle\left(a_{\mathrm{L}, \omega^{\prime}} e^{-i\left(k x+\omega^{\prime} t\right)}+a_{\mathrm{R}, \omega^{\prime}} e^{-i\left(-k x+\omega^{\prime} t\right)}-\text { H.c. }\right)\right. \\
& \left.\times\left(a_{\mathrm{L}, \omega^{\prime \prime}} e^{-i k x}+a_{\mathrm{R}, \omega^{\prime \prime}} e^{i k x}-\mathrm{H} . \mathrm{c} .\right)\right\rangle \\
= & \frac{\hbar Z_{0}}{4 \pi} \int_{-\infty}^{\infty} \mathrm{d} t e^{i \omega t} \int_{0}^{\infty} \mathrm{d} \omega^{\prime} \sqrt{\omega^{\prime}} \int_{0}^{\infty} \mathrm{d} \omega^{\prime \prime} \sqrt{\omega^{\prime \prime}} 2 e^{-i \omega^{\prime} t} \delta\left(\omega^{\prime}-\omega^{\prime \prime}\right) \\
= & \frac{\hbar Z_{0}}{2 \pi} \int_{0}^{\infty} \mathrm{d} \omega^{\prime} \omega^{\prime} 2 \pi \delta\left(\omega-\omega^{\prime}\right)=Z_{0} \hbar \omega,
\end{aligned}
$$

where we assumed negligible temperature such that the only contribution from the expectation value is terms on the form $\left\langle a a^{\dagger}\right\rangle=1$. The result shows that the left- and right-travelling modes each contribute $\hbar \omega / 2$ to the power spectral density $S_{V V}[\omega] / Z_{0}$, which agrees well with our expectations for the quantum vacuum fluctuations. 


\subsubsection{Mirrors and resonators}

We can now proceed to introduce boundary conditions in the open transmission line. Grounding one end at $x=0$ gives the boundary condition $\Phi(0, t)=0$; it is equivalent to inserting a mirror in open space. It is also possible to connect one end of the transmission line to ground via a capacitance or via a SQUID (the latter gives a tunable boundary condition, a moving mirror, as discussed in Sec. 1.2).

A semi-infinite transmission line still has a continuum of modes, but the boundary condition gives rise to a mode structure. This can be seen as an interference effect between waves approaching the mirror and waves that have been reflected off the mirror. In Paper VIII, we explore this mode structure, which is imposed also on the vacuum fluctuations, by placing an artificial atom close to a mirror and varying its resonance frequency. The relaxation rate of the atom is proportional to the spectral density of the voltage fluctuations at the atom transition frequency, which given the boundary condition $\Phi(0, t)=0$ becomes $S_{V V}[\omega] / Z_{0}=2 \hbar \omega \sin ^{2}(k x)$.

A semi-infinite transmission line is also used in the experiment of Paper III, where a three-level artificial atom placed close to a mirror is used to mediate photon-photon interactions. Here, the main point of using the mirror is to give unidirectionality that improves efficiency; all the photons must go out in one direction, whereas in an open transmission line they can be scattered by the atom in two different directions.

If we introduce boundary conditions at two points $x=0$ and $x=d$ in an open transmission line, we create a resonator. Using Eq. (2.21) to satisfy $\Phi(0, t)=0=\Phi(d, t)$, we see that these boundary conditions enforce $a_{\mathrm{L}, \omega}=-a_{\mathrm{R}, \omega}$ and $\sin (k x)=0$. Thus, only modes with frequencies

$$
\omega_{n}=\frac{n \pi v}{d}=\frac{n \pi}{d \sqrt{L_{0} C_{0}}},
$$

where $n$ is an integer, remain [178]. We now have a discrete, yet still infinite, collection of harmonic oscillators $[177,178]$. In most applications only the fundamental mode $\omega_{1} \equiv \omega_{\mathrm{r}}$ is used, giving the well-known harmonic oscillator Hamiltonian

$$
H=\hbar \omega_{\mathrm{r}}\left(a^{\dagger} a+\frac{1}{2}\right),
$$

where $a$ now is the annihilation operator for this localized mode.

In Papers I and IV, we consider setups with such resonators coupled to artificial atoms. The next section shows how an artificial atom can 


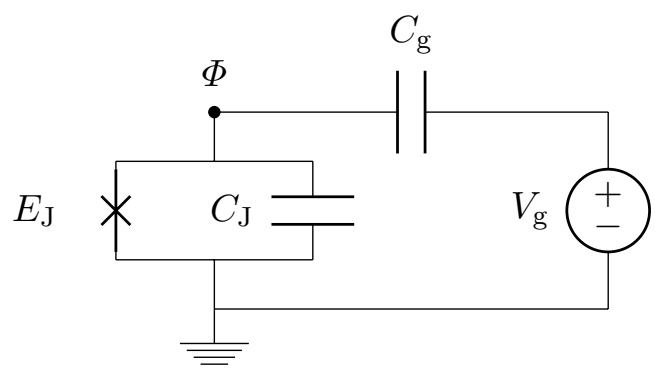

Figure 2.3: Circuit diagram for a Cooper-pair box. The Josephson junction is modelled by the capacitance $C_{\mathrm{J}}$ in parallel with a nonlinear inductor having Josephson energy $E_{\mathrm{J}}$. The node between the gate capacitance $C_{\mathrm{g}}$ and the Josephson junction is called the "island".

be constructed from superconducting circuits, and Sec. 2.5 explores the Hamiltonian that results from the interaction between an atom and a resonator.

\subsection{The transmon qubit}

There are several ways to build an artificial atom with superconducting circuits $[27,178,184-190]$. Their common denominator is the use of Josephson junctions to provide a nonlinear element. In this section, we will give an overview of one implementation, the transmon [173], which is used or considered in all the appended papers of this thesis.

The transmon is a variation on the Cooper-pair box (CPB) [185, 186, 191], the circuit diagram of which is shown in Fig. 2.3. The CPB consists of a small superconducting island connected to a superconducting reservoir via a Josephson junction, which allows Cooper pairs to tunnel on and off the island. The model also includes an external voltage source $V_{\mathrm{g}}$ coupled to the island via a gate capacitance $C_{\mathrm{g}}$, to determine the background charge $n_{\mathrm{g}}=C_{\mathrm{g}} V_{\mathrm{g}} / 2 e$ (measured in units of Cooper pairs) that the environment induces on the island.

Using Eqs. (2.7) and (2.12) we get the CPB Lagrangian

$$
\mathfrak{L}_{\mathrm{CPB}}=\frac{C_{\mathrm{g}}\left(\dot{\Phi}-V_{\mathrm{g}}\right)^{2}}{2}+\frac{C_{\mathrm{J}} \dot{\Phi}^{2}}{2}-E_{\mathrm{J}}\left(1-\cos \frac{2 e \Phi}{\hbar}\right)
$$

Applying the Legendre transformation, identifying the conjugate momentum (the node charge) $Q=\left(C_{\mathrm{J}}+C_{\mathrm{g}}\right) \dot{\Phi}-C_{\mathrm{g}} V_{\mathrm{g}}$, and removing constant 

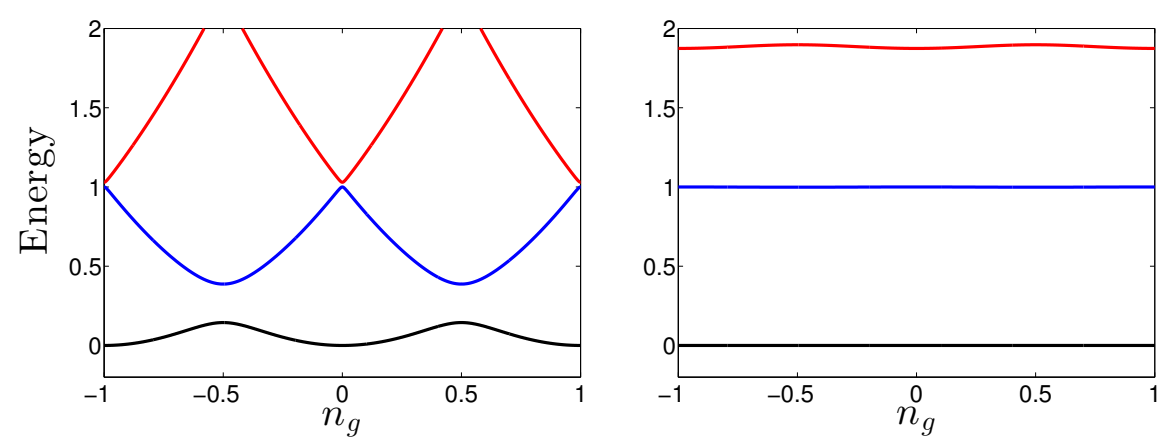

Figure 2.4: The three lowest energy levels of a CPB plotted as a function of $n_{\mathrm{g}}$ for $E_{\mathrm{J}} / E_{\mathrm{C}}=1$ (left) and $E_{\mathrm{J}} / E_{\mathrm{C}}=20$ (right). The energy scale is normalized to the level separation between the ground state and the first excited state at $n_{\mathrm{g}}=0$. The decreased sensitivity to charge noise in the transmon regime $\left(E_{\mathrm{J}} / E_{\mathrm{C}} \gg 1\right)$, as well as the decreased anharmonicity, is apparent.

terms that do not contribute to the dynamics, we arrive at the Hamiltonian

$$
H_{\mathrm{CPB}}=4 E_{\mathrm{C}}\left(n-n_{\mathrm{g}}\right)^{2}-E_{\mathrm{J}} \cos \phi,
$$

where $E_{\mathrm{C}}=e^{2} / 2\left(C_{\mathrm{g}}+C_{\mathrm{J}}\right)$ is the electron charging energy, $n=-Q / 2 e$ is the number of Cooper pairs on the island, and $\phi=2 e \Phi / \hbar$.

We now promote $\Phi$ and $Q$ to operators in the same way as in the previous sections. This translates into a commutation relation for $n$ and $\phi$, which since the Hamiltonian is periodic in $\phi$ should be expressed as $[105$, 179]

$$
\left[e^{i \phi}, n\right]=e^{i \phi} .
$$

From this follows that $e^{ \pm i \phi}|n\rangle=|n \mp 1\rangle$, where $|n\rangle$ is the charge basis counting the number of Cooper pairs. Using the resolution of unity [104] and $\cos \phi=\left(e^{i \phi}+e^{-i \phi}\right) / 2$ we can then write the Hamiltonian in the charge basis as $[177,178]$

$$
H_{\mathrm{CPB}}=\sum_{n}\left[4 E_{\mathrm{C}}\left(n-n_{\mathrm{g}}\right)^{2}|n\rangle\langle n|-\frac{1}{2} E_{\mathrm{J}}(|n+1\rangle\langle n|+| n-1\rangle\langle n|)\right] .
$$

The energy level structure of $H_{\mathrm{CPB}}$ depends on the parameters $E_{\mathrm{J}}, E_{\mathrm{C}}$, and $n_{\mathrm{g}}$. As $n_{\mathrm{g}}$ represents the influence of the environment, we would like it to have little effect in order to have a stable, controllable system. This is achieved when $E_{\mathrm{J}} \gg E_{\mathrm{C}}$ (the phase rather than the charge dominates), as 
is illustrated in Fig. 2.4. The price to be paid is a decrease in anharmonicity, i.e., the difference between the transition energy needed to go from the ground state to the first excited state and the transition energy needed to go from the first excited state to the second excited state. To work as a qubit, an artificial atom has to be anharmonic enough to be approximated as a two-level system when driving the first transition; a signal driving the atom from the ground state to the first excited state should not be able to induce a further transition to the second excited state. Fortunately, the influence of $n_{\mathrm{g}}$ decays much faster than the anharmonicity when $E_{\mathrm{J}}$ is increased, so the transmon can indeed be used as a qubit [173].

In the limit $E_{\mathrm{J}} \gg E_{\mathrm{C}}$, the energy levels of the transmon are approximately given by [173]

$$
E_{m}=-E_{\mathrm{J}}+\sqrt{8 E_{\mathrm{J}} E_{\mathrm{C}}}\left(m+\frac{1}{2}\right)-\frac{E_{\mathrm{C}}}{12}\left(6 m^{2}+6 m+3\right) .
$$

From this, we get the anharmonicity

$$
\alpha=E_{2,1}-E_{1,0}=\left(E_{2}-E_{1}\right)-\left(E_{1}-E_{0}\right)=-E_{C} .
$$

Since $E_{\mathrm{C}} \ll E_{\mathrm{J}}$, the anharmonicity is small compared to the transition frequencies, but it can still be large enough compared to drive strengths and relaxation rates in the system to ensure that the transmon can be operated as a two-level system. To achieve a low $E_{\mathrm{C}}$, one adds a shunt capacitance in parallel with $C_{\mathrm{J}}$, often by designing the transmon in the form of two islands that form an interdigitated finger structure with high capacitance. The islands are usually connected by a SQUID (see Sec. 1.2) rather than a single Josepshon junction. The SQUID functions as a Josephson junction with a tunable $E_{\mathrm{J}}$ (controlled by the magnetic flux through the SQUID), which means that the energy levels and transition frequencies of the transmon can be tuned in situ during an experiment.

The transmon is not always operated as a pure two-level system. In some cases, the second excited state of the transmon is actually used to implement qubit gates $[162,192,193]$. Indeed, $d$-level systems, qudits, make quantum computation possible with less resources [194-196] and can simulate more quantum systems than qubits [197]; transmons seem well suited to work as qudits $[198,199]$. Another advantage of the transmon is that the superconducting island shape can be designed to couple to several other transmons or resonators [200, 201]. 


\subsection{Surface acoustic waves}

While most papers in this thesis are concerned with transmons coupled to photons in electric transmission lines, Paper VI shows that a transmon can also interact with phonons in the form of surface acoustic waves. In this section, we will first review classical theory for SAWs and then show how they couple to a transmon.

\subsubsection{Classical SAW theory}

Surface acoustic waves are a type of vibrations in a solid that are confined to the surface of the material, decaying exponentially in the bulk. Such solutions to the wave equation in a 3D material were first found by Lord Rayleigh in 1885 [202] and they are important in many natural phenomena, e.g, in earthquakes. About 50 years ago, it was realized that SAWs in piezoelectric materials can be used to convert long-wavelength electromagnetic radiation to short-wavelength vibrations, which has proven extremely useful in TV and cellphone technology [93-96]. Here, we will mainly follow Ref. [93] to explain the basic mechanisms.

Applying a force $\mathbf{F}$ to a $3 \mathrm{D}$ solid material can give rise to particle displacements $\mathbf{u}$. To describe this, one defines the stress tensor

$$
T_{i j}=\frac{F_{i}}{A_{j}}, \quad i, j=x, y, z,
$$

where $F_{i}$ is the force in direction $i$ and $A_{j}$ is the cross-section area in direction $j$ (the area vector is taken as the normal pointing outwards from the volume under consideration). The stress gives rise to a strain

$$
S_{i j}=\frac{\partial u_{i}}{\partial j}, \quad i, j=x, y, z,
$$

which measures the fractional change of length in the material. The stress and the strain are related by an elasticity tensor, or stiffness coefficient,

$$
T_{i j}=c_{i j k l} S_{k l} .
$$

From symmetry considerations it is possible to show that $T_{i j}=T_{j i}$, reducing the number of independent stresses to six. These can be gathered in a vector

$$
\mathbf{T}=\left(T_{x x}, T_{y y}, T_{z z}, T_{y z}, T_{z x}, T_{x y}\right) .
$$


Similarly, we can define a strain vector $\mathbf{S}$, where the last three entries are symmetrizations,

$$
\mathbf{S}=\left(S_{x x}, S_{y y}, S_{z z}, S_{y z}+S_{z y}, S_{x z}+S_{z x}, S_{x y}+S_{y x}\right) .
$$

We are then left with

$$
\mathbf{T}=c \mathbf{S},
$$

where $c$ is a $6 \times 6$ stiffness matrix.

For a dielectric material, there is a similar relation between an applied electric field $\mathbf{E}$ and the electrical displacement $\mathbf{D}$,

$$
\mathbf{D}=\varepsilon \mathbf{E},
$$

where $\varepsilon$ is a $3 \times 3$ permittivity matrix. In most materials, the processes of Eqs. (2.37) and (2.38) are independent of each other. However, in a piezoelectric material, the arrangement of the atoms is such that a strain will give rise to a polarization charge, and vice versa. The result is that we get two coupled equations,

$$
\begin{aligned}
& \mathbf{T}=c \mathbf{S}-e^{\mathrm{T}} \mathbf{E}, \\
& \mathbf{D}=e \mathbf{S}+\varepsilon \mathbf{E},
\end{aligned}
$$

where $e$ is a $3 \times 6$ matrix known as the piezoelectric constant and $e^{\mathrm{T}}$ is its transpose. To see the interplay between electricity and vibrations, we will consider as an example a compressional wave moving in the $x$ direction in a material with $e_{11} \neq 0$. Eqs. (2.39)-(2.40) then become

$$
\begin{aligned}
T_{1} & =c_{11} S_{1}-e_{11} E_{1}, \\
D_{1} & =e_{11} S_{1}+\varepsilon_{11} E_{1} .
\end{aligned}
$$

Following Ref. [93], we will now connect to Sec. 2.2, showing that this example is equivalent to a transmission line. To see this, we first introduce the particle displacement velocity $v=\partial_{t} u$ (we drop subscripts from here on). Since $S=\partial_{x} u$, taking the time derivative of Eq. (2.41) and rearranging the terms gives

$$
\frac{\partial v}{\partial x}=\frac{1}{c} \frac{\partial T}{\partial t}+\frac{e}{c} \frac{\partial E}{\partial t} .
$$

Then, solving Eq. (2.39) for $S$, inserting the result into Eq. (2.40), solving the resulting equation for $E$ and inserting that result in Eq. (2.43) leads to

$$
\frac{\partial v}{\partial x}=\frac{1}{c^{\prime}} \frac{\partial T}{\partial t}+\frac{e}{\varepsilon c^{\prime}} \frac{\partial D}{\partial t},
$$


where we have defined

$$
c^{\prime}=c+\frac{e^{2}}{\varepsilon} \equiv c\left(1+K^{2}\right) .
$$

$K^{2}=e^{2} / \varepsilon c$ is called the electromechanical coupling; it is a defining property for piezoelectric materials. In the most strongly piezoelectric materials, like lithium niobate $\left(\mathrm{LiNbO}_{3}\right), K^{2} \approx 5 \cdot 10^{-2}$, while gallium arsenide (GaAs), which was used in the experiment of Paper VI, has $K^{2} \approx 7 \cdot 10^{-4}$.

In the quasi-electrostatic approximation, $D$ is constant (assuming that the material has no free charges and that there is external voltage applied). With this and the definition in Eq. (2.32), Eq. (2.44) becomes

$$
\frac{\partial v}{\partial x}=\frac{1}{c^{\prime} A} \frac{\partial F}{\partial t}
$$

for a cross-section area $A$.

To get a second equation that will help us connect this to a transmission line model, we consider the effect of stress on an infinitesimal cube with sides $\mathrm{d} x, \mathrm{~d} y$, and $\mathrm{d} z$, having mass density $\rho_{m}$. On one side of the cube, there is a force $T \mathrm{~d} y \mathrm{~d} z$, while on the opposite side the force is $\left(T+\left(\partial_{x} T\right) \mathrm{d} x\right) \mathrm{d} y \mathrm{~d} z$. Newton's second law, $\mathbf{F}=m \mathbf{a}$, used on the net force gives

$$
\frac{\partial T}{\partial x} \mathrm{~d} x \mathrm{~d} y \mathrm{~d} z=\rho_{m} \mathrm{~d} x \mathrm{~d} y \mathrm{~d} z \frac{\partial v}{\partial t},
$$

which can be rewritten as

$$
\frac{\partial F}{\partial x}=\rho_{m} A \frac{\partial v}{\partial t}
$$

Eqs. (2.46) and (2.48) are of the same form as the equations for voltage $V$ and current $I$ in a transmission line,

$$
\begin{aligned}
& \frac{\partial V}{\partial x}=-L_{0} \frac{\partial I}{\partial t} \\
& \frac{\partial I}{\partial x}=-C_{0} \frac{\partial V}{\partial t} .
\end{aligned}
$$

Making the identifications

$$
V \leftrightarrow-F, \quad I \leftrightarrow v, \quad L_{0} \leftrightarrow \rho_{m} A, \quad C_{0} \leftrightarrow \frac{1}{c^{\prime} A},
$$




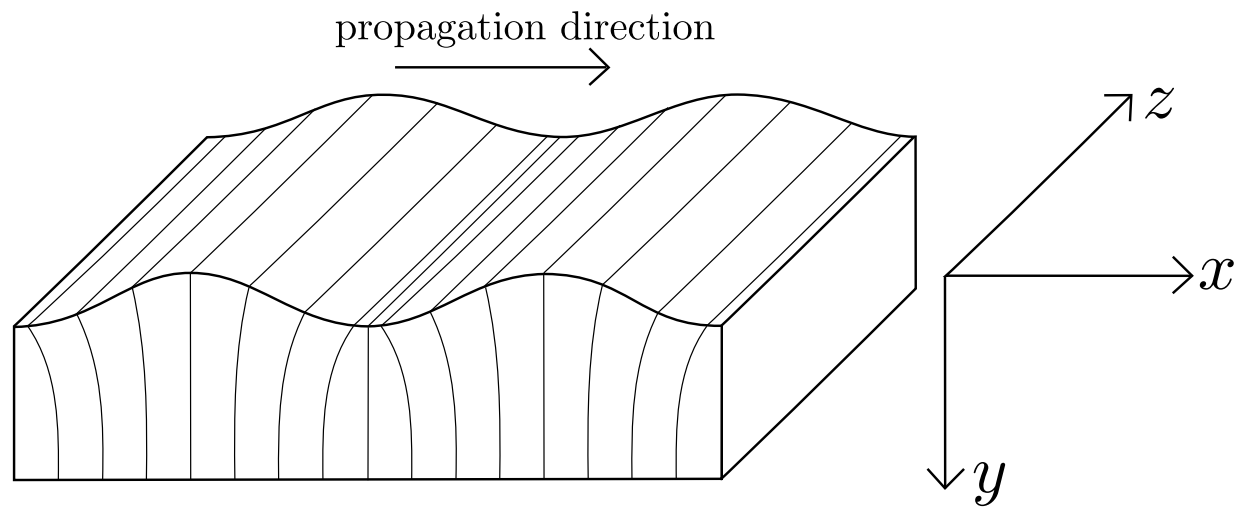

Figure 2.5: SAW propagation on a substrate. The particle motion includes both compression in the $x$ direction, which is the propagation direction of the SAW, and shearing in the $y$ direction.

we can thus extract the acoustic wave propagation velocity

$$
v_{\text {wave }}=\frac{1}{\sqrt{L_{0} C_{0}}}=\frac{1}{\sqrt{\rho_{m} A \frac{1}{c^{\prime} A}}}=\sqrt{\frac{c^{\prime}}{\rho_{m}}} .
$$

The example above was for an acoustic wave moving in the bulk of a piezoelectric material. A surface acoustic wave is more complicated, as shown in Fig. 2.5. If we let $x$ be the propagation direction (the surface is the $x z$ plane), the SAW will include compressional motion in the $x$ direction and shearing in the $y$ direction (this gives in total elliptical particle motion), along with an electrostatic wave. Since we are interested in connecting to electronics, it is convenient to make the electric potential at the surface, $\phi$, our main variable. Given $\phi, u_{x}$ and $u_{y}$ will be fixed by material parameters.

The full SAW description involves permittivities and piezoelectric couplings in several directions as well as an exponentially decaying part in the $y$ direction. With the reasonable approximations that the compressional motion dominates and that the electrostatic part is described by the constant potential $\phi$ in a shallow layer at the surface (zero elsewhere), it is still possible to use a transmission line picture. The potential $\phi$ is then equated to the voltage $V$ in the transmission line and a transmission line conductance $Y_{0}$ is defined such that the total power carried by the SAW, including both electrical and mechanical contributions, is

$$
P=Y_{0}|\phi|^{2} .
$$




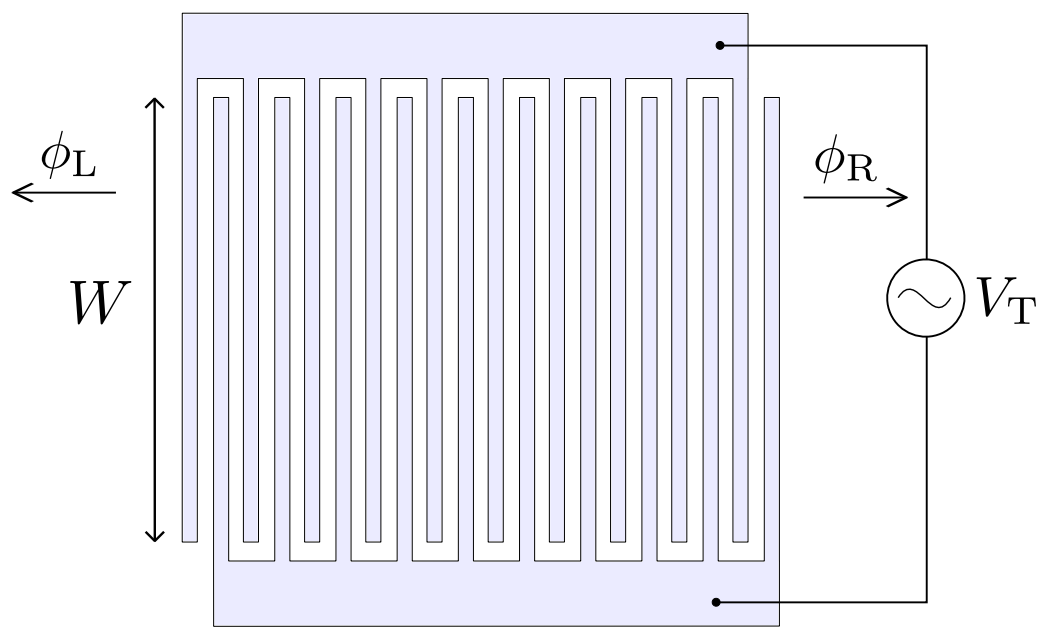

Figure 2.6: A sketch of an IDT. Two islands with periodically spaced metal fingers of length $W$ are connected to a voltage source $V_{\mathrm{T}}$. The voltage induces strain in the piezoelectric substrate, launching SAWs to the left and right with electric potential $\phi_{\mathrm{L} / \mathrm{R}}$.

Since the conductance will depend on the width $W$ of the SAW, a characteristic conductance $y_{0}$ is defined using the SAW wavelength $\lambda$,

$$
y_{0}=\frac{\lambda}{W} Y_{0}
$$

From calculations similar to those of the example above, but with some more care taken to reflect that we are now at a surface, it can be shown that [93]

$$
y_{0}=\frac{2 \pi C_{\mathrm{s}} v_{0}}{K^{2}}
$$

where $v_{0}$ is the SAW propagation velocity and $C_{\mathrm{s}}=\varepsilon_{0}+\varepsilon_{\mathrm{p}}\left(\varepsilon_{\mathrm{p}}\right.$ being the permittivity of the substrate and $\varepsilon_{0}$ the permittivity of the medium above the substrate).

With a theory for SAW propagation in place, the next step is to generate the waves. Current SAW technology is based on the interdigital transducer (IDT), invented in 1965 [203]. An IDT consists of a number of metallic fingers placed periodically on the piezoelectric substrate as sketched in Fig. 2.6. When an $\mathrm{AC}$ voltage $V_{\mathrm{T}}$ is applied to the transducer, it induces strain in the piezoelectric substrate and generates SAWs with amplitude $\phi=\mu V_{\mathrm{T}}$, where $\mu$ is a coupling constant that will be determined 
shortly. Conversely, a SAW wave arriving at the IDT structure will generate a current $I=g_{m} \phi$. The reciprocity between conversion from electrical signal to SAW and vice versa leads to a relation between $g_{m}$ and $\mu$ [93]:

$$
g_{m}=2 \mu Y_{0},
$$

where the factor 2 comes from the applied voltage generating waves with amplitude $\mu V_{\mathrm{T}}$ in each propagation direction (both to the left and to the right).

To calculate $\mu$ for a single IDT finger pair of length $W$, we can approximate it as a capacitor with capacitance $W C_{s}$ and a uniform charge density set by $V_{T}$. This acts as a current source in the SAW transmission line; the result is [93]

$$
\mu=i c_{\mathrm{g}} K^{2},
$$

where $c_{\mathrm{g}}$ is a geometry factor on the order of 1 . Its exact value depends on the metallization ratio $\eta$, the ratio between the finger width $a$ and the inter-finger distance $p$. The result in Eq. (2.57) also assumes that we are considering a frequency matching the resonance condition $\lambda=2 p$.

For the case of multiple fingers, one simply sums the individual finger contributions, including the phase shift the SAW acquires travelling from one finger to the next. If we, for convenience, ground one of the electrodes and let the coordinates of the $N_{\mathrm{p}}$ fingers of the other electrode be $x_{k}$, we get

$$
|\mu|=c_{\mathrm{g}} K^{2}\left|\sum_{k=1}^{N_{\mathrm{p}}} e^{i 2 \pi f x_{k} / v_{0}}\right| .
$$

If the fingers are equally spaced such that $\left|x_{k}-x_{k-1}\right|=\lambda=v_{0} / f_{0}$, the result for an arbitrary frequency $f$ is

$$
|\mu(f)|=c_{\mathrm{g}} K^{2} \frac{\sin \left(N_{\mathrm{p}} \pi \frac{f-f_{0}}{f_{0}}\right)}{\sin \left(\pi \frac{f-f_{0}}{f_{0}}\right)},
$$

which has the peak value $N_{\mathrm{p}} K^{2}$ on resonance, $f=f_{0}$. The possibility to choose finger spacings that couple preferentially to certain frequencies is part of the reason why SAWs are widely used in filtering applications.

For a compact description of the IDT functions, it is useful to develop an equivalent circuit model, shown in Fig. 2.7. The conversion of electrical signal to SAW is represented by a real-valued acoustic admittance $G_{\mathrm{a}}$. 

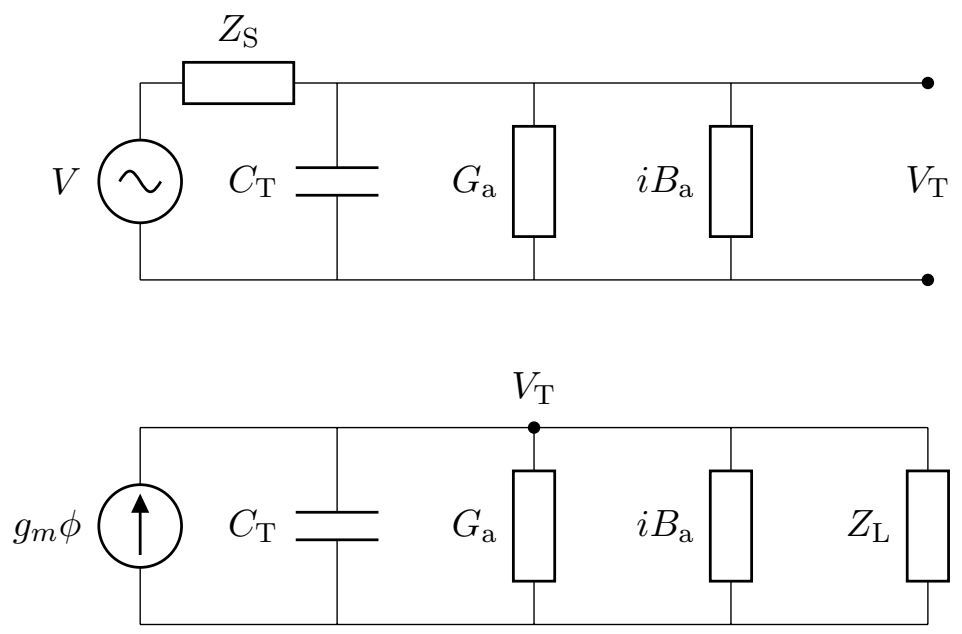

Figure 2.7: Circuit models for transmitter and receiver IDTs. Top: a transmitter IDT where a voltage $V$ is applied through a source impedance $Z_{\mathrm{S}}$, resulting in a voltage $V_{\mathrm{T}}$ over the IDT which consists of the capacitance $C_{\mathrm{T}}$ between the two electrodes and a complex acoustic admittance $G_{\mathrm{a}}+i B_{\mathrm{a}}$. Bottom: a receiver IDT. Here, the incoming SAW amplitude $\phi$ acts as a current source in the circuit, which includes a load impedance $Z_{\mathrm{L}}$.

Since the power lost through such a circuit element, $\frac{1}{2}\left|V_{\mathrm{T}}\right|^{2} G_{\mathrm{a}}$, should equal the emitted SAW power, $2 \frac{1}{2}|\phi|^{2} Y_{0}$, we get

$$
G_{\mathrm{a}}=2|\mu(f)|^{2} Y_{0}=-\mu g_{m}
$$

There is also an imaginary-valued acoustic admittance $i B_{\mathrm{a}}$, which arises from the fact that SAWs generated at one finger can be picked up again by another finger. It turns out that $B_{\mathrm{a}}$ is the Hilbert transform of $G_{\mathrm{a}}$ [93],

$$
B_{\mathrm{a}}(f)=\frac{1}{\pi} \mathcal{P} \int_{-\infty}^{\infty} \mathrm{d} f^{\prime} \frac{G_{\mathrm{a}}\left(f^{\prime}\right)}{f^{\prime}-f}
$$

where $\mathcal{P}$ denotes principal value. For the case of equally spaced IDT fingers, we have $B_{\mathrm{a}}\left(f_{0}\right)=0$. In the equivalent circuit model, the capacitance $C_{\mathrm{T}}$ between the two electrodes is also included. When the IDT picks up SAWs instead of emitting them, it is represented by a current source with $I=g_{m} \phi$. 


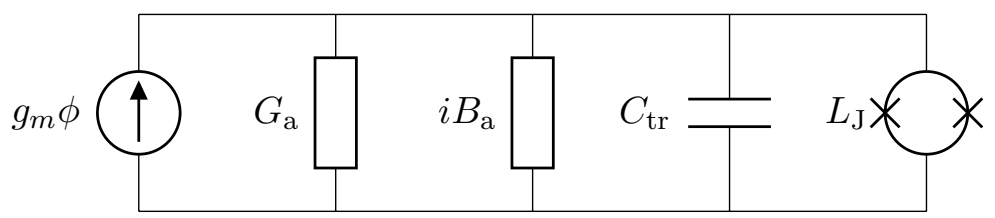

Figure 2.8: Circuit model of a transmon coupled to SAWs. The transmon is approximated as an $L C$-oscillator. The fingers of the transmon shunt capacitance also serve as an IDT structure that connects to the SAWs.

\subsubsection{Coupling SAWs to a transmon}

In the previous subsection, we saw that SAWs can be described in terms of a moving electric potential in a transmission line model. The difference compared to the photons in the purely electrical transmission line of Sec. 2.2 is that the SAWs are mainly vibrations, the quanta of which are phonons. We can also observe that the transmon of Sec. 2.3 couples to charge and includes a large shunt capacitance, which is often designed in an interdigitated finger structure similar to that of IDTs. It thus seems possible to couple a transmon to SAWs, realizing quantum optics experiments with slow-moving phonons instead of fast photons. This idea was first presented in Refs. $[167,204]$ and realized in Paper VI.

To estimate the coupling we can get between the SAW phonons and a transmon, we can consider the semiclassical circuit model shown in Fig. 2.8, as is done in the appendix of Paper VI. Here, the capacitance $C_{\text {tr }}$ between the two electrodes is that of the transmon. The transmon SQUID is included in the form of a nonlinear inductance $L_{\mathrm{J}}$, which from the Josephson equations, Eqs. (2.9) and (2.10), becomes

$$
L_{\mathrm{J}}=\frac{\hbar}{2 e I_{\mathrm{C}} \cos \phi},
$$

where now $\phi$ is the phase difference across the SQUID as defined in Sec. 2.1 and $e$ is the elementary charge. In the semiclassical approximation, we just consider a single excitation in the transmon and can thus neglect the nonlinearity of $L_{\mathrm{J}}(\phi \ll 1)$, such that the resonance frequency of the transmon is

$$
\omega_{\mathrm{tr}}=\frac{1}{\sqrt{L_{\mathrm{J}} C_{\mathrm{tr}}}} .
$$

To make things easier, we also assume that we are on acoustic resonance, such that $B_{\mathrm{a}}=0$ and does not affect $\omega_{\mathrm{tr}}$. 
The damping factor of a parallel $R L C$ circuit is given by

$$
\zeta=\frac{1}{2 R} \sqrt{\frac{L}{C}},
$$

so the rate at which the transmon relaxes to phonons is

$$
\Gamma=\omega_{\mathrm{tr}} \frac{G_{\mathrm{a}}}{2} \sqrt{\frac{L_{\mathrm{J}}}{C_{\mathrm{tr}}}}=N_{\mathrm{p}} c_{\mathrm{g}}^{2} K^{2} \omega_{\mathrm{tr}}
$$

where $N_{\mathrm{p}}$ is the number of finger pairs. Here, we used Eqs. (2.54), (2.55), (2.60), and (2.63), together with $|\mu|=N_{\mathrm{p}} c_{\mathrm{g}} K^{2}, C_{\mathrm{tr}}=N_{\mathrm{p}} W C_{\mathrm{s}}$, and $2 \pi v_{0} / \lambda=\omega_{\text {tr }}$.

The result in Eq. (2.65) changes slightly if we use different finger structures (e.g., pairs of double fingers which can minimize problems with purely mechanical reflections) or another metallization ratio, but the main result stands: with a few fingers, depending on piezoelectric substrate, we can get a relatively fast relaxation from the qubit to phonons. For example, in Paper VI $N_{\mathrm{p}}=20$ finger pairs were used on a GaAs substrate with $K^{2}=7 \cdot 10^{-4}$, which resulted in a relaxation rate to phonons of $\Gamma / 2 \pi=38 \mathrm{MHz}$. Indeed, for a strongly piezoelectric substrate and many IDT fingers, it even seems possible to reach a regime of ultrastrong coupling, where $\Gamma$ is on the order of $\omega_{\mathrm{tr}}$.

From a quantum optics perspective, one of the main reasons that the transmon coupled to SAWs is a very interesting system is that it forms a "giant artificial atom". Natural atoms used in traditional quantum optics typically have a radius $r \approx 10^{-10} \mathrm{~m}$ and interact with light at optical wavelengths $\lambda \approx 10^{-7}-10^{-6} \mathrm{~m}[18,169]$. Sometimes the atoms are excited to high Rydberg states $\left(r \approx 10^{-8}-10^{-7} \mathrm{~m}\right)$, but they then interact with microwave radiation $\left(\lambda \approx 10^{-3}-10^{-1} \mathrm{~m}\right)[3,19]$. Microwaves also interact with superconducting qubits, but even these structures are typically measured in micrometers (although some recent designs approach wavelength sizes [205]). Consequently, theoretical investigations of atom-light interaction usually rely on the dipole approximation that the atom can be considered point-like when compared to the light wavelength. This is clearly not the case for the transmon coupled to SAWs, since here each IDT finger is a connection point and the separation between fingers is always on the order of wavelengths.

Inspired by the SAW-transmon setup, Paper VII investigates the physics of an atom coupled to an open transmission line at a number of discrete 


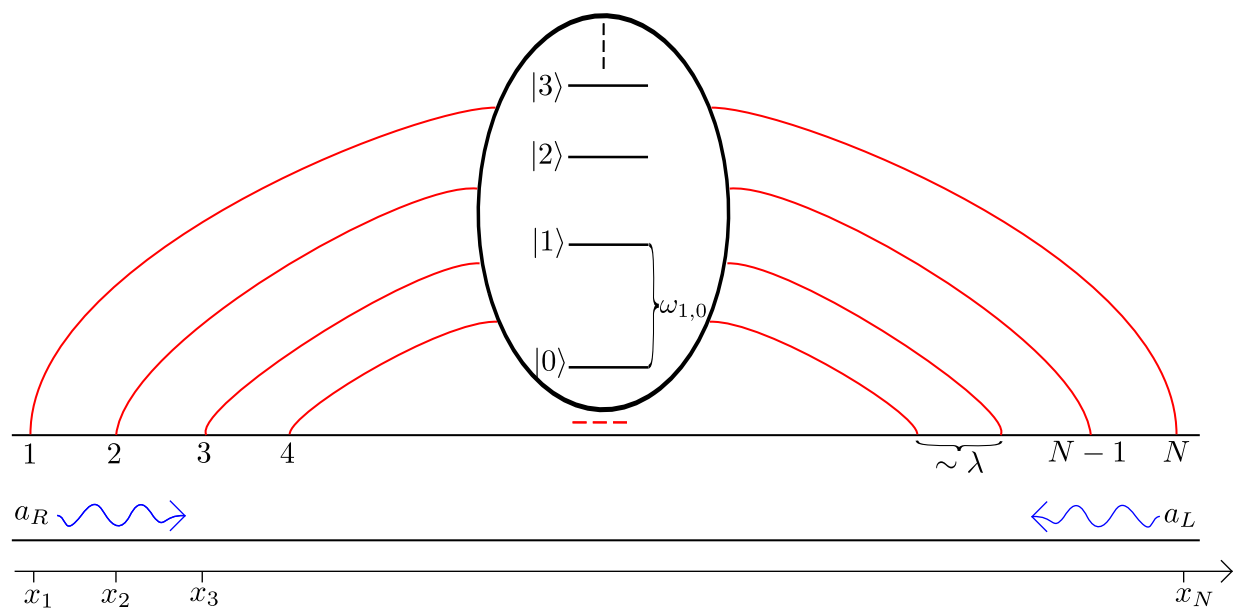

Figure 2.9: A schematic model for a giant artificial multi-level atom, connected at $N$ points to left- and right-moving excitations in a $1 \mathrm{D}$ transmission line.

points, which can be spaced wavelengths apart. A sketch of this model is shown in Fig. 2.9. While this is a model of the SAW-transmon setup, approximating each finger as having a negligible width, it should also be possible to realize with a variation of the transmon design, the "xmon" [200], coupled to the usual superconducting transmission line considered in Sec. 2.2. The Hamiltonian for our model is

$$
H=H_{\text {atom }}+H_{\mathrm{TL}}+H_{\text {int }},
$$

where we define the multi-level-atom Hamiltonian

$$
H_{\text {atom }}=\sum_{m} \omega_{m}|m\rangle\langle m|
$$

the transmission line Hamiltonian

$$
H_{\mathrm{TL}}=\sum_{j} \omega_{j}\left(a_{\mathrm{R} j}^{\dagger} a_{\mathrm{R} j}+a_{\mathrm{L} j}^{\dagger} a_{\mathrm{L} j}\right)
$$

and the interaction Hamiltonian

$$
H_{\mathrm{int}}=\sum_{j, k, m} g_{j k m}\left(\sigma_{-}^{m}+\sigma_{+}^{m}\right)\left(a_{\mathrm{R} j} e^{-i \omega_{j} x_{k} / v}+a_{\mathrm{L} j} e^{i \omega_{j} x_{k} / v}+\text { H.c. }\right),
$$

where $\sigma_{-}^{m}=|m\rangle\langle m+1|$ and $\sigma_{+}^{m}=|m+1\rangle\langle m|$. The atom has energy levels $m=0,1,2, \ldots$ with energies $\omega_{m}$ (for brevity and simplicity, we will here 
and in the rest of the thesis work in units where $\hbar=1$ ). It is connected to right- and left-moving modes $\mathrm{R} j$ and $\mathrm{L} j$ of the transmission line with some coupling strength $g_{j k m}$ at $N$ points with coordinates $x_{k}$. We assume that the time it takes for a transmission line excitation to travel with velocity $v$ across all the atom connection points is negligible compared to the timescale of atom relaxation. Thus, only the phase shifts between connection points need to be included in the calculations, not the time delays.

The derivation of results such as relaxation rates of the atom requires techniques that will be introduced in Chapter 3. Here, we will just briefly state the results, which are closely connected to the classical theory for IDTs in the previous subsection. Firstly, the relaxation rate becomes proportional to $\left|\sum_{k=1}^{N} e^{i \omega_{j} x_{k} / v}\right|^{2}$, just like the acoustic admittance $G_{\mathrm{a}} \propto|\mu|^{2}$ (see Eq. (2.58)). Thus, we can design our atom such that it only relaxes fast at certain transition frequencies, but remains protected from decay at others. Secondly, the atom transition frequencies are shifted by a small amount (a Lamb shift), approximately proportional to the Hilbert transform of $\left|\sum_{k=1}^{N} e^{i \omega_{j} x_{k} / v}\right|^{2}$. This is similar to the imaginary acoustic admittance $i B_{\mathrm{a}}$, which shifts the $L C$ resonance frequency in the semiclassical calculation above if the atom is not on resonance with the IDT structure.

The theoretical and experimental work on SAW-transmon and other giant atom systems have only just started with Papers VI and VII. In Chapter 7, we will give some possible directions for future work.

\subsection{The Jaynes-Cummings model}

With our building blocks for quantum optics in place, it is time to look closer at how they can be combined. An important and common setup is that of one or several atoms coupled to a resonator, illustrated for superconducting circuits in Fig. 2.10. The system consisting of a resonator and one two-level atom (qubit) can be described by the Rabi Hamiltonian [206]

$$
H_{\mathrm{Rabi}}=\omega_{\mathrm{r}} a^{\dagger} a+\frac{\omega_{\mathrm{a}}}{2} \sigma_{z}+g \sigma_{x}\left(a+a^{\dagger}\right),
$$

where $\omega_{\mathrm{r}}$ is the frequency of the resonator, $\omega_{\mathrm{a}}$ is the transition frequency of the atom, $g$ is the strength of the coupling between the atom and the resonator, $a\left(a^{\dagger}\right)$ is the annihilation (creation) operator for the resonator 


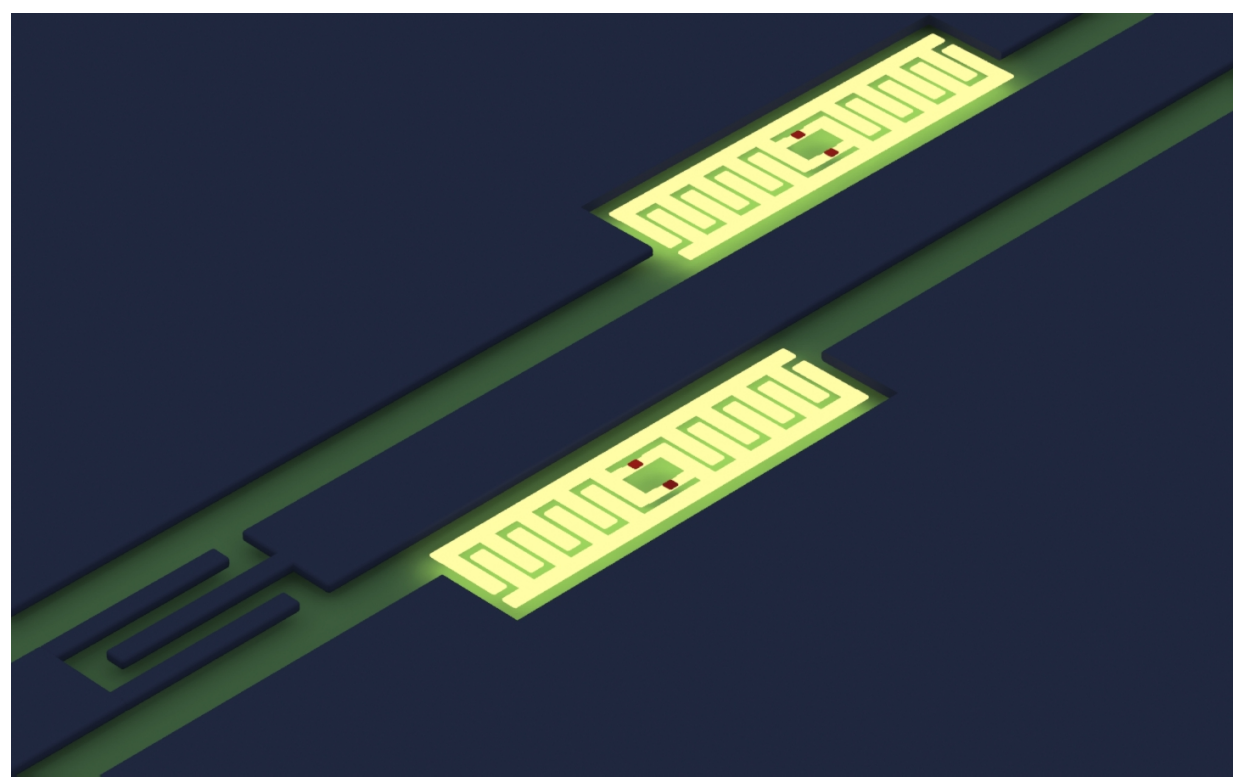

Figure 2.10: An artist's rendering of a transmission line resonator coupled to two transmons. The dark blue line in the center is the center conductor of the transmission line, interrupted by a capacitance to the left. The large dark blue areas are ground planes. The golden sawtooth shapes form the large capacitances of the two transmons (needed to achieve $E_{\mathrm{J}} / E_{\mathrm{C}} \gg 1$ ) and the red dots are the Josephson junctions (two junctions, instead of one, are used in a SQUID configuration to allow tuning of $E_{\mathrm{J}}$ with an external magnetic field). Illustration by Philip Krantz (krantznanoart.com).

mode, and the $\sigma_{i}$ are Pauli matrices describing the atom. $\sigma_{x}$ can be rewritten as $\sigma_{+}+\sigma_{-}$, where $\sigma_{-}\left(\sigma_{+}\right)$is the lowering (raising) operator of the atom.

In the last part of Eq. (2.70), we have the terms $a \sigma_{-}$and $a^{\dagger} \sigma_{+}$, which will oscillate with frequency $\omega_{\mathrm{r}}+\omega_{\mathrm{a}}$ in the interaction picture. Provided that this frequency is much larger than $g$, which sets the relevant timescale for the system dynamics, these terms will average out and we can neglect them in our calculations. This approximation is known as the rotating wave approximation (RWA) [207]. It reduces the Rabi Hamiltonian to the Jaynes-Cummings Hamiltonian [105, 174, 208]

$$
H_{\mathrm{JC}}=\omega_{\mathrm{r}} a^{\dagger} a+\frac{\omega_{\mathrm{a}}}{2} \sigma_{z}+g\left(a \sigma_{+}+a^{\dagger} \sigma_{-}\right) .
$$

In this simpler model, the number of excitations in the system is con- 
served, allowing for an explicit solution of the Hamiltonian (solving the Rabi Hamiltonian is also possible, but it is a much harder problem [209]). It is straightforward to extend the Jaynes-Cummings model to include more than two levels of the atom, as is often necessary when dealing with the transmon [173], or to include more atoms; the latter case is called the Tavis-Cummings model [210]. The only added complication is that the photons need not couple equally strongly to different transitions or atoms. In Paper IV, we study and explain experimental results in such a system, where a multi-level transmon is coupled to a superconducting resonator. There, we also apply drives to both the resonator and the transmon.

To understand the results of Paper IV, the concept of dressed states $[105,211]$ is vital. When the interaction $g$ between atom and resonator in Eq. (2.71) is turned on, the eigenstates of the system are no longer "bare" excitations of either resonator or atom, but instead dressed states that mix atom and resonator excitations. Diagonalizing Eq. (2.71) gives the eigenstates [105]

$$
\begin{aligned}
& |n,+\rangle=\cos \left(\frac{\varphi_{n}}{2}\right)|e\rangle|n\rangle+\sin \left(\frac{\varphi_{n}}{2}\right)|g\rangle|n+1\rangle, \\
& |n,-\rangle=-\sin \left(\frac{\varphi_{n}}{2}\right)|e\rangle|n\rangle+\cos \left(\frac{\varphi_{n}}{2}\right)|g\rangle|n+1\rangle,
\end{aligned}
$$

where $|n\rangle$ is the bare resonator state with $n$ photons, $|g / e\rangle$ is the bare atom ground/excited state, and the angle $\varphi_{n}$ is given by

$$
\tan \left(\varphi_{n}\right)=\frac{\Omega_{n}}{\Delta} .
$$

Here, $\Delta=\omega_{\mathrm{a}}-\omega_{\mathrm{r}}$ is the detuning between atom and resonator, and

$$
\Omega_{n}=\sqrt{\Delta^{2}+4 g^{2}(n+1)}
$$

is the splitting between the dressed states. The energies of the dressed states are given by

$$
E(n, \pm)=\left(n+\frac{1}{2}\right) \omega_{r} \pm \frac{1}{2} \Omega_{n} .
$$

The transition from bare to dressed states is illustrated in Fig. 2.11. If one adds a drive to the system, as in Paper IV, this can be interpreted as "dressing the dressed states", giving doubly dressed states. 

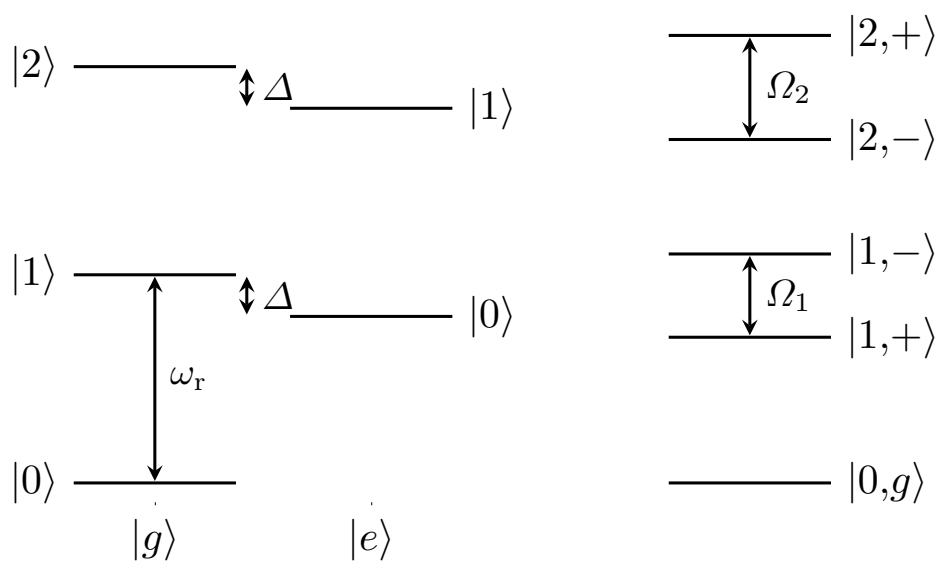

Figure 2.11: Level diagrams for bare and dressed states in the Jaynes-Cummings model. To the left, the bare states are represented in two ladders, one for $|g\rangle$ and one for $|e\rangle$. The states $|g\rangle n+1$ and $|e\rangle n$ are close in energy, separated by $\Delta$, but there are no transitions possible between the two. To the right, the coupling $g$ is switched on and the nearby states hybridize, forming a new ladder with different level spacings.

A useful twist on the Jaynes-Cummings model is the dispersive regime, which is when the detuning $|\Delta|$ is much larger than the coupling strength $g$. In this case, we can apply the unitary transformation

$$
U_{\text {disp }}=\exp \left[\lambda\left(a \sigma_{+}-a^{\dagger} \sigma_{-}\right)\right],
$$

where $\lambda=g / \Delta$, to Eq. (2.71), and do a perturbation expansion in the small parameter $\lambda$. We defer the details of this calculation to Appendix A. Keeping terms up to first order in $\lambda$, the result is

$$
H_{\mathrm{disp}}=\left(\omega_{\mathrm{r}}+\chi \sigma_{z}\right) a^{\dagger} a+\frac{\omega_{\mathrm{a}}+\chi}{2} \sigma_{z},
$$

where $\chi=g^{2} / \Delta$. Perturbation to higher orders in $\lambda$ is possible [212] and the procedure can also be carried out for a multi-level atom [173].

The most important feature of the Hamiltonian in Eq. (2.78) is that the atom and the resonator no longer interact by exchanging excitations. Instead, changing the state of the qubit will shift the resonator frequency and changing the number of photons in the resonator will shift the qubit frequency. Both these effects have been used to perform QND measurements 
of qubit states $[24,213]$ and photon number $[8,214]$. The measurements we consider in Paper I are all based on using a coherent signal to probe a resonator dispersively coupled to one or two qubits.

Another advantage of the dispersive regime is that it allows us to trace out the resonator degrees of freedom to achieve an effective description for just the atom $[165,215,216]$. This procedure is an integral part of the work in Paper I. It reduces the number of degrees of freedom in the system from infinity to two and lets us see clearly what back-action a measurement on the resonator has on the qubit.

As superconducting circuits for quantum optics have been developed, it has been realized that they make it possible to reach the regime of ultrastrong coupling, where $g \gtrsim 0.1 \omega_{\mathrm{r} / \mathrm{a}}$ [217-220] (strong coupling, on the other hand, is when $g$ is greater than the rate of relaxation to the environment). In this regime, the RWA breaks down, and either the full Rabi Hamiltonian or some better approximation must be used [207]. Ultrastrong coupling has been experimentally demonstrated in the last few years both for optical photons interacting with electrons in quantum wells [221], with natural molecules [222], in superconducting circuits [223, 224], in a twodimensional electron gas (2DEG) [225, 226], and with magnons [227]. As we mention in the outlook for Papers VI and VII, it should also be possible to reach this interesting regime with SAWs coupled to a transmon.

While we use the Jaynes-Cummings model in Papers I and IV, in the rest of the appended papers we deal with one or several artificial atoms coupled to an infinite or semi-infinite transmission line. Experiments in these kinds of systems have only recently reached high quality in superconducting circuits [35-41]; theoretical studies of one [183, 228-240] or several atoms [241-251] have been done for a longer time, recently also for the case of ultrastrong coupling $[219,252,253]$. The crucial difference compared to the Jaynes-Cummings model is that the artificial atom here couples to a continuum of modes, which can transport excitations away from the atom. To study these systems, we need to use master equations and input-output theory, which is the topic of the next chapter. 



\section{Chapter 3}

\section{Master equations and input-output theory}

For a closed quantum system, a system which does not interact in any way with its surroundings, the Hamiltonian $H$ of the system gives us all the information we need to derive its time evolution. We can either describe the dynamics in the Schrödinger picture, where the state of the system, $|\psi\rangle$, evolves in time according to the Schrödinger equation [104]

$$
\frac{\mathrm{d}}{\mathrm{d} t}|\psi\rangle=-i H|\psi\rangle,
$$

and system operators are constant, or in the Heisenberg picture, where the state is constant and a system operator $c$ evolves according to the Heisenberg equation [104]

$$
\dot{c}=-i[c, H] .
$$

Another, more general, way to describe the state and its evolution in the Schrödinger picture is to use the density matrix $\rho=\sum_{i} w_{i}\left|\psi_{i}\right\rangle\left\langle\psi_{i}\right|$, which represents an ensemble of states (many experiments) with $w_{i}$ giving the probability of the system being in the state $\left|\psi_{i}\right\rangle$. The time evolution for $\rho$ is given by the Liouville-von Neumann equation

$$
\dot{\rho}=-i[H, \rho],
$$

which is easily derived from the Schrödinger equation [104]. The density matrix representation is the most convenient one when we open up the system to influence from the outside. Given $\rho$, one can compute expectation values from $\langle c\rangle=\operatorname{tr}(c \rho)$, and the time evolution is given by $\partial_{t}\langle c\rangle=\operatorname{tr}(c \dot{\rho})$. 
In this chapter, we will start to study open quantum systems, i.e., quantum systems that are coupled to a surrounding environment, often called a bath. We will first derive a master equation, which gives an effective description of the system density matrix, including the effects of interaction with the bath but tracing out the bath degrees of freedom. We then consider input-output theory for the quantum system, and develop a stochastic description of the bath effects, which will be useful in later chapters.

\subsection{Master equation for an open quantum system}

The problem we face when considering an open quantum system is one of size: our system couples to an environment which consists of infinitely many other quantum systems. To find an effective description of only the system itself, we need to make a number of approximations, which fortunately are well justified in many experiments. The procedure is well described in many books $[116,254,255]$; here, we will mainly follow the treatment in the last one.

To illustrate the derivation of a master equation, we consider a model where our quantum system is a single two-level atom, coupled to a bath consisting of an infinite number of harmonic oscillators of different resonance frequencies. The Hamiltonian is

$$
\begin{aligned}
H & =H_{\text {atom }}+H_{\text {bath }}+H_{\text {int }}, \\
H_{\text {atom }} & =\frac{\omega_{\mathrm{a}}}{2} \sigma_{z}, \\
H_{\text {bath }} & =\sum_{j} \omega_{j} b_{j}^{\dagger} b_{j}, \\
H_{\text {int }} & =\sum_{j} g_{j}\left(b_{j}+b_{j}^{\dagger}\right)\left(\sigma_{-}+\sigma_{+}\right),
\end{aligned}
$$

Note that we do not make the RWA on the interaction Hamiltonian, even though we will assume that the coupling $g_{j}$ is weak. It will become clear later that making the RWA too early in the master equation derivation eliminates essential terms.

To simplify calculations, we move to the interaction picture,

$$
\tilde{X}(t)=e^{i\left(H_{\text {atom }}+H_{\text {bath }}\right) t} X e^{-i\left(H_{\text {atom }}+H_{\text {bath }}\right) t},
$$


for all operators $X$. The equation for the time evolution of the transformed density matrix of the total system (atom plus bath), can then be written

$$
\dot{\tilde{\rho}}_{\text {tot }}(t)=-i\left[\tilde{H}_{\mathrm{int}}(t), \tilde{\rho}_{\mathrm{tot}}(t)\right] .
$$

The solution of this equation is

$$
\tilde{\rho}_{\text {tot }}(t)=\tilde{\rho}_{\text {tot }}(0)-i \int_{0}^{t} \mathrm{~d} \tau\left[\tilde{H}_{\text {int }}(\tau), \tilde{\rho}_{\text {tot }}(\tau)\right],
$$

which can be inserted back into Eq. (3.9). If we then trace over the bath degrees of freedom, the result is an equation for the effective atom density matrix $\rho$,

$$
\dot{\tilde{\rho}}(t)=\operatorname{tr}_{\text {bath }}\left(-i\left[\tilde{H}_{\text {int }}(t), \tilde{\rho}_{\text {tot }}(0)\right]-\int_{0}^{t} \mathrm{~d} \tau\left[\tilde{H}_{\text {int }}(t),\left[\tilde{H}_{\text {int }}(\tau), \tilde{\rho}_{\text {tot }}(\tau)\right]\right]\right) .
$$

Every step leading up to Eq. (3.11) has been exact. To go further, we need two approximations. First is the Born approximation, which relies on the coupling $g_{j}$ to be weak and the bath to be large compared to the atom. With these conditions fulfilled, it is reasonable to assume that the bath density matrix $\rho_{\text {bath }}$ does not change significantly due to the interaction with the atom. We also assume the atom and the bath to be in a separable state initially; this means that we have $\rho_{\text {tot }}(t) \approx \rho(t) \otimes \rho_{\text {bath }}$.

The second approximation is the Markov approximation, which states that the bath has no memory, i.e., the interaction between the atom and bath is so weak, and the bath is so large, that any imprint the atom makes on the bath at time $t^{\prime}$ does not come back and affect the dynamics of the atom at a later time $t$. This allows us to replace $\rho(\tau)$ with $\rho(t)$ in Eq. (3.11). All this, together with $\left\langle H_{\text {int }}\right\rangle_{\text {bath }}=0$, reduces Eq. (3.11) to

$$
\dot{\tilde{\rho}}(t)=-\int_{0}^{t} \mathrm{~d} \tau \operatorname{tr}_{\text {bath }}\left(\left[\tilde{H}_{\text {int }}(t),\left[\tilde{H}_{\text {int }}(\tau), \tilde{\rho}(t) \tilde{\rho}_{\text {bath }}\right]\right]\right) .
$$

To proceed from Eq. (3.12) is not hard, only somewhat tedious. The details of the calculations have therefore been deferred to Appendix B. Once the trace over the bath has been taken, the RWA has been applied, terms have been collected, and the transformation back from the interaction picture has been done, the final result can be compactly expressed as

$$
\dot{\rho}=-i\left[\frac{\omega_{\mathrm{a}}^{\prime}}{2} \sigma_{z}, \rho\right]+\Gamma \mathcal{D}\left[\sigma_{-}\right] \rho
$$


Here, $\Gamma$ is the atom relaxation rate, given by

$$
\Gamma=2 \pi J\left(\omega_{\mathrm{a}}\right) g^{2}\left(\omega_{\mathrm{a}}\right)
$$

where $J(\omega)$ is the bath density of states and $g\left(\omega_{j}\right)=g_{j}$. The superoperator $\mathcal{D}[X] \rho=X \rho X^{\dagger}-\frac{1}{2} X^{\dagger} X \rho-\frac{1}{2} \rho X^{\dagger} X$ in Eq. (3.13) is said to be on the Lindblad form [256]. It should be noted that the form of the terms in the master equation ensures that the density matrix properties $\rho=\rho^{\dagger}$, $\operatorname{tr}(\rho)=1$, and $\rho>0$ are preserved.

In the master equation, we also have a new atom transition frequency

$$
\omega_{\mathrm{a}}^{\prime}=\omega_{\mathrm{a}}+\mathcal{P} \int_{0}^{\infty} \mathrm{d} \omega J(\omega) g^{2}(\omega)\left(\frac{1}{\omega+\omega_{\mathrm{a}}}-\frac{1}{\omega-\omega_{\mathrm{a}}}\right) .
$$

The difference compared to the isolated atom is a Lamb shift. If we had made the RWA too early, the first term in the integral would be missing $[257,258]$.

In the derivation of Eq. (3.13), we assumed negligible temperature, i.e., $\left\langle b_{j}^{\dagger} b_{j}\right\rangle_{\text {bath }}=0$, but it is also possible to include a finite temperature $T$, giving

$$
\left\langle b_{j}^{\dagger} b_{j}\right\rangle_{\mathrm{bath}}=\frac{1}{e^{\hbar \omega_{j} / k_{\mathrm{B}} T}-1} \equiv \bar{n}\left(\omega_{j}, T\right),
$$

where $k_{\mathrm{B}}$ is Boltzmann's constant. The result is a modification of Eq. (3.13) to

$$
\dot{\rho}=-i\left[\frac{\omega_{\mathrm{a}}^{\prime \prime}}{2} \sigma_{z}, \rho\right]+\left(1+\bar{n}\left(\omega_{\mathrm{a}}, T\right)\right) \Gamma \mathcal{D}\left[\sigma_{-}\right] \rho+\bar{n}\left(\omega_{\mathrm{a}}, T\right) \Gamma \mathcal{D}\left[\sigma_{+}\right] \rho,
$$

where we now see that the thermal excitations in the bath can excite the atom via the $\mathcal{D}\left[\sigma_{+}\right] \rho$ term. Furthermore, the Lamb shift also gets thermal contributions, leading to the new atom transition frequency $\omega_{\mathrm{a}}^{\prime \prime}$. The expression for this new frequency is a more complicated version of Eq. (3.15), including terms with $\bar{n}(\omega, T)$ in the integral.

Master equations are used in all the appended papers; to interact with the quantum systems we want to study, we have to connect them to the outside world. From a purely theoretical viewpoint, the master equation derivation in Paper VII is the most interesting, since it is made for a giant artificial atom, a case which does not seem to have been considered previously. 


\subsection{Input-output theory}

While the master equation gives us an effective description of the quantum system that is coupled to an environment, it does not immediately tell us what the output of the system is, or how that output depends on some input. This is the topic of input-output theory. Let us study again the example of the previous section, with a two-level atom coupled to a bath of harmonic oscillators. The Hamiltonian (slightly rewritten for convenience which becomes clear during the calculations) is

$$
\begin{aligned}
H & =H_{\text {atom }}+H_{\text {bath }}+H_{\text {int }}, \\
H_{\text {atom }} & =\frac{\omega_{a}}{2} \sigma_{z}, \\
H_{\text {bath }} & =\int_{0}^{\infty} \mathrm{d} \omega \omega b^{\dagger}(\omega) b(\omega), \\
H_{\text {int }} & =i \int_{0}^{\infty} \mathrm{d} \omega \sqrt{\frac{\Gamma(\omega)}{2 \pi}}\left(b^{\dagger}(\omega) \sigma_{-}-\sigma_{+} b(\omega)\right),
\end{aligned}
$$

where $\Gamma(\omega)$ is a frequency-dependent coupling strength. Here, we have already made the RWA on the interaction Hamiltonian $H_{\text {int }}$.

To study input and output from the system, we follow the treatment in Ref. [259]. We begin by extending the lower integration limits to $-\infty$ in Eqs. (3.20) and (3.21). This is an acceptable approximation, since only terms with frequencies close to $\omega_{a}$ are important. With this modification done, we write down the Heisenberg equation for the atom and bath operators. Using the commutation relations $\left[\sigma_{-}, \sigma_{+}\right]=-\sigma_{z},\left[\sigma_{-}, \sigma_{z}\right]=2 \sigma_{-}$, and $\left[b(\omega), b^{\dagger}\left(\omega^{\prime}\right)\right]=\delta\left(\omega-\omega^{\prime}\right)$ gives

$$
\begin{aligned}
\dot{b}(\omega) & =-i \omega b(\omega)+\sqrt{\frac{\Gamma(\omega)}{2 \pi}} \sigma_{-} \\
\dot{\sigma}_{-} & =-i \omega_{\mathrm{a}} \sigma_{-}+\sigma_{z} \int_{-\infty}^{\infty} \mathrm{d} \omega \sqrt{\frac{\Gamma(\omega)}{2 \pi}} b(\omega) .
\end{aligned}
$$

Defining $b_{0}(\omega)=b\left(\omega, t=t_{0}\right)$ as the initial bath state at some initial time $t_{0}<t$ lets us write the solution to the first equation as

$$
b(\omega)=e^{-i \omega\left(t-t_{0}\right)} b_{0}(\omega)+\sqrt{\frac{\Gamma(\omega)}{2 \pi}} \int_{t_{0}}^{t} \mathrm{~d} t^{\prime} \sigma_{-}\left(t^{\prime}\right) e^{-i \omega\left(t-t^{\prime}\right)} .
$$


Now we make the Markov approximation that $\Gamma(\omega)$ varies slowly around $\omega_{a}$ and thus can be taken to be constant in this region, $\Gamma(\omega)=\Gamma$. This gives the idealized description of white noise, which is delta-correlated (the damping of the atom at time $t$ will only depend on the bath at time $t$, not on the bath at any previous time). We also define the in-field

$$
b_{\text {in }}(t)=\frac{1}{\sqrt{2 \pi}} \int_{-\infty}^{\infty} \mathrm{d} \omega e^{-i \omega\left(t-t_{0}\right)} b_{0}(\omega) .
$$

Inserting the expression for $b(\omega)$ from Eq. (3.24) into Eq. (3.23) with this addition gives, after a little algebra, that the equation for $\sigma_{-}$can be written

$$
\dot{\sigma}_{-}=-i \omega_{\mathrm{a}} \sigma_{-}+\sqrt{\Gamma} \sigma_{z} b_{\mathrm{in}}(t)+\frac{\Gamma}{2} \sigma_{z} \sigma_{-} .
$$

From this we see two things. Firstly, the in-field from the bath will affect the evolution of the atom operator $\sigma_{-}$. Secondly, even if there is no input from the bath, the coupling will still give rise to damping of $\sigma_{-}$(since $\sigma_{z} \sigma_{-}=-\sigma_{-}$) with a rate which we recognize as the dephasing rate $\Gamma / 2$, half of the relaxation rate for an atomic excitation.

Another way to solve the Heisenberg equation for $b(\omega)$ is to define $b_{1}(\omega)=b\left(\omega, t=t_{1}\right)$ as the future bath state at some future time $t_{1}>t$. This gives

$$
b(\omega)=e^{-i \omega\left(t-t_{1}\right)} b_{1}(\omega)-\sqrt{\frac{\Gamma(\omega)}{2 \pi}} \int_{t}^{t_{1}} \mathrm{~d} t^{\prime} \sigma_{-}\left(t^{\prime}\right) e^{-i \omega\left(t-t^{\prime}\right)},
$$

and defining the out-field

$$
b_{\text {out }}(t)=\frac{1}{\sqrt{2 \pi}} \int_{-\infty}^{\infty} \mathrm{d} \omega e^{-i \omega\left(t-t_{1}\right)} b_{1}(\omega)
$$

lets us write the time-reversed equation for $\sigma_{-}$as

$$
\dot{\sigma}_{-}=-i \omega_{\mathrm{a}} \sigma_{-}+\sqrt{\Gamma} \sigma_{z} b_{\mathrm{out}}(t)-\frac{\Gamma}{2} \sigma_{z} \sigma_{-} .
$$

Using that Eqs. (3.26) and (3.29) should give the same result at time $t$ now gives the important result

$$
b_{\text {out }}(t)=b_{\text {in }}(t)+\sqrt{\Gamma} \sigma_{-}(t),
$$

which connects input and output, the goal of this section. 


\subsection{Quantum stochastic calculus}

As was mentioned in Sec. 1.3, there is a deep connection between stochastic fluctuations in an environment and dissipation from a system coupled to that environment [116-119]. With the results from the previous sections of the current chapter, we can now develop a description of the bath which emphasizes its stochastic character. This description, quantum stochastic calculus, will be very useful when we later deal with more advanced inputoutput scenarios (in particular, measurements on a quantum system).

In the previous section, we defined the in-field $b_{\text {in }}(t)$ in Eq. (3.25). Setting $t_{0}=0$ for simplicity from here on, we can calculate the commutation relation

$$
\begin{aligned}
{\left[b_{\text {in }}(t), b_{\text {in }}^{\dagger}(t)\right] } & =\frac{1}{2 \pi} \int_{-\infty}^{\infty} \mathrm{d} \omega \int_{-\infty}^{\infty} \mathrm{d} \omega^{\prime} e^{-i \omega t+i \omega^{\prime} t^{\prime}}\left[b_{0}(\omega), b_{0}^{\dagger}\left(\omega^{\prime}\right)\right] \\
& =\frac{1}{2 \pi} \int_{-\infty}^{\infty} \mathrm{d} \omega e^{-i \omega\left(t-t^{\prime}\right)}=\delta\left(t-t^{\prime}\right),
\end{aligned}
$$

which strengthens the view of $b_{\text {in }}(t)$ as white noise. We define $[116,259]$

$$
B_{t}=\int_{0}^{t} \mathrm{~d} s b_{\text {in }}(s),
$$

which is known as a quantum Wiener process. From this we get the quantum noise increments

$$
\mathrm{d} B_{t}=\int_{t}^{t+\mathrm{d} t} \mathrm{~d} s b_{\text {in }}(s),
$$

which is often written in differential form as $\mathrm{d} B_{t}=b_{\text {in }}(t) \mathrm{d} t$.

Now we can write down the time evolution operator $U(t) \equiv U(t, 0) \equiv$ $U_{t}$, which is defined to evolve a state from time 0 to time $t$ as $|\psi(t)\rangle=$ $U(t)|\psi(0)\rangle$ in the Schrödinger picture or to evolve an operator $X$ in the Heisenberg picture as $X(t)=U^{\dagger}(t) X(0) U(t)$. Using the Schrödinger equation and the Hamiltonian in Eqs. (3.18)-(3.21), we get, in the rotating frame of $H_{\text {bath }}$,

$$
\begin{aligned}
U(t)= & \mathcal{T} \exp \left[-i \int_{0}^{t} \mathrm{~d} t^{\prime}\left(H_{\text {atom }}+i \sqrt{\frac{\Gamma}{2 \pi}}\right.\right. \\
& \left.\left.\times \int_{-\infty}^{\infty} \mathrm{d} \omega\left(b^{\dagger}(\omega) \sigma_{-} e^{i \omega t^{\prime}}-\sigma_{+} b(\omega) e^{-i \omega t^{\prime}}\right)\right)\right] \\
= & \mathcal{T} \exp \left[-i H_{\text {atom }} t+\sqrt{\Gamma}\left(B_{t}^{\dagger} \sigma_{-}-\sigma_{+} B_{t}\right)\right]
\end{aligned}
$$


where $\mathcal{T}$ is the time-ordering operator. Note that we here have replaced the $b$ 's with $b_{0}$ 's to get the final result.

We would like to expand Eq. (3.34) for a small increment in $t$ to get $\mathrm{d} U_{t}$. However, care is required when dealing with stochastic increments. There are two approaches to the problem: Stratonovich calculus and Itō calculus [116, 259-262].

In Stratonovich calculus, an integral of some function or system operator $f(t)$ with stochastic increments is defined as

$$
\int_{0}^{t} f\left(t^{\prime}\right) \mathrm{d} B_{t^{\prime}}=\lim _{n \rightarrow \infty} \sum_{j=0}^{n} \frac{1}{2}\left(f\left(t_{j}\right)+f\left(t_{j+1}\right)\right)\left(B_{t_{j+1}}-B_{t_{j}}\right) .
$$

The advantage of this approach is that the chain rule of ordinary calculus continues to hold for stochastic increments, i.e., $\mathrm{d}(A B)=A(\mathrm{~d} B)+(\mathrm{d} A) B$. The downside is that the terms in the sum defining the integral don't necessarily commute, as the terms are defined on overlapping time intervals.

In Itō calculus, the same integral is instead defined as

$$
\int_{0}^{t} f\left(t^{\prime}\right) \mathrm{d} B_{t^{\prime}}=\lim _{n \rightarrow \infty} \sum_{j=0}^{n} f\left(t_{j}\right)\left(B_{t_{j+1}}-B_{t_{j}}\right),
$$

which ensures that the terms in the sum commute, as they only overlap on a time interval of length zero $\left(f\left(t_{j}\right)\right.$ only depends on $B_{t}$ with $\left.t<t_{j}\right)$. The price to pay is that the chain rule has to be modified to $\mathrm{d}(A B)=$ $A(\mathrm{~d} B)+(\mathrm{d} A) B+(\mathrm{d} A)(\mathrm{d} B)$. In the following we will work with Itō calculus, since the fact that $f(t) \mathrm{d} B_{t}$ commute makes calculating expectation values easier.

Before we can find $\mathrm{d} U_{t}$, we need to calculate expectation values such as

$$
\begin{aligned}
\left\langle\mathrm{d} B_{t} \mathrm{~d} B_{t}^{\dagger}\right\rangle & =\int_{t}^{t+\mathrm{d} t} \mathrm{~d} t^{\prime} \int_{t}^{t+\mathrm{d} t} \mathrm{~d} t^{\prime \prime}\left\langle 0\left|b_{\mathrm{in}}\left(t^{\prime}\right) b_{\mathrm{in}}^{\dagger}\left(t^{\prime \prime}\right)\right| 0\right\rangle \\
& =\int_{t}^{t+\mathrm{d} t} \mathrm{~d} t^{\prime} \int_{t}^{t+\mathrm{d} t} \mathrm{~d} t^{\prime \prime}\left\langle 0\left|\left[b_{\mathrm{in}}\left(t^{\prime}\right), b_{\mathrm{in}}^{\dagger}\left(t^{\prime \prime}\right)\right]\right| 0\right\rangle \\
& =\int_{t}^{t+\mathrm{d} t} \mathrm{~d} t^{\prime} \int_{t}^{t+\mathrm{d} t} \mathrm{~d} t^{\prime \prime} \delta\left(t^{\prime}-t^{\prime \prime}\right)\langle 0 \mid 0\rangle \\
& =\int_{t}^{t+\mathrm{d} t} \mathrm{~d} t^{\prime}=\mathrm{d} t,
\end{aligned}
$$

where we have used $b_{\text {in }}(t)|0\rangle=0$ in the first equality. This tells us that in vacuum expectation values we can treat $\mathrm{d} B_{t} \mathrm{~d} B_{t}^{\dagger}$ as $\mathrm{d} t$, which explains 
why we need to keep the second-order term in the chain rule. Calculations similar to Eq. (3.37) give that $\mathrm{d} B_{t} \mathrm{~d} B_{t}, \mathrm{~d} B_{t}^{\dagger} \mathrm{d} B_{t}^{\dagger}$, and $\mathrm{d} B_{t}^{\dagger} \mathrm{d} B_{t}$ are all 0. Together, these results are known as the Itō rules for vacuum expectation values, and they show why $B_{t}$ is called a quantum Wiener process. The Itō rules can be generalized to having the bath in a thermal state [116, 259].

Expanding Eq. (3.34) using Itō calculus now gives

$$
\begin{aligned}
\mathrm{d} U_{t}= & {\left[-i H_{\text {atom }} \mathrm{d} t+\sqrt{\Gamma}\left(\sigma_{-} \mathrm{d} B_{t}^{\dagger}-\sigma_{+} \mathrm{d} B_{t}\right)\right.} \\
& \left.+\frac{1}{2}\left(-i H_{\text {atom }} \mathrm{d} t+\sqrt{\Gamma}\left(\sigma_{-} \mathrm{d} B_{t}^{\dagger}-\sigma_{+} \mathrm{d} B_{t}\right)\right)^{2}+\mathcal{O}\left(t^{3 / 2}\right)\right] U_{t} \\
= & {\left[-i H_{\text {atom }} \mathrm{d} t+\left(L \mathrm{~d} B_{t}^{\dagger}-L^{\dagger} \mathrm{d} B_{t}\right)-\frac{1}{2} L^{\dagger} L \mathrm{~d} t+\mathcal{O}\left(t^{3 / 2}\right)\right] U_{t}, }
\end{aligned}
$$

where we have introduced the common notation $L=\sqrt{\Gamma} \sigma_{-}$. With this expression for the time evolution in hand, we can now also derive the Itō Langevin equation for an atom operator $c[116,259]$,

$$
\begin{aligned}
\mathrm{d} c(t)= & \mathrm{d}\left(U_{t}^{\dagger} c U_{t}\right)=\mathrm{d} U_{t}^{\dagger} c U_{t}+U_{t}^{\dagger} c \mathrm{~d} U_{t}+\mathrm{d} U_{t}^{\dagger} c \mathrm{~d} U_{t} \\
= & -i\left[c(t), H_{\text {atom }}(t)\right] \mathrm{d} t+\mathcal{D}^{\dagger}[L] c(t) \mathrm{d} t \\
& +[c(t), L(t)] \mathrm{d} B_{t}^{\dagger}+\left[L^{\dagger}(t), c(t)\right] \mathrm{d} B_{t},
\end{aligned}
$$

where $\mathcal{D}^{\dagger}[L] c=L^{\dagger} c L-\frac{1}{2}\left\{L^{\dagger} L, c\right\}$, and we have used the Itō rules for vacuum expectation values.

To calculate expectation values of atom operators, we can either stay in the Heisenberg picture and use Eq. (3.39), or we can find an effective density matrix for the atom in the Schrödinger picture. To do the latter, we note that

$$
\begin{aligned}
\langle c(t)\rangle & =\operatorname{tr}_{\text {atom }} \operatorname{tr}_{\text {bath }}\left[\left(c(t) \otimes \mathbf{1}_{\text {bath }}\right)\left(\rho_{\text {atom }}(0) \otimes \rho_{\text {bath }}(0)\right)\right] \\
& =\operatorname{tr}_{\text {atom }} \operatorname{tr}_{\text {bath }}\left[U^{\dagger}(t)\left(c(0) \otimes \mathbf{1}_{\text {bath }}\right) U(t)\left(\rho_{\text {atom }}(0) \otimes \rho_{\text {bath }}(0)\right)\right],
\end{aligned}
$$

where $\mathbf{1}_{\text {bath }}$ is the identity operator in the bath Hilbert space. We also assume that the atom+bath state factorizes at time $t=0$ as $\left|\psi_{0}\right\rangle \otimes|0\rangle$, where $\left|\psi_{0}\right\rangle$ is some pure atom state. By using the cyclic property of the trace, we can then rewrite this equation as

$$
\langle c(t)\rangle=\operatorname{tr}_{\text {atom }}[c(0) \rho(t)],
$$


where $\rho(t)$ is the effective atom density matrix

$$
\rho(t)=\operatorname{tr}_{\text {bath }}\left[U(t)\left(\rho_{\text {atom }}(0) \otimes \rho_{\text {bath }}(0)\right) U^{\dagger}(t)\right] .
$$

From the Itō Langevin equation for $c$, Eq. (3.39), we get

$$
\begin{aligned}
\langle\mathrm{d} c(t)\rangle & =\left\langle\psi_{0}, 0|\mathrm{~d} c(t)| \psi_{0}, 0\right\rangle \\
& =\left\langle\psi_{0}\left|-i\left[c(t), H_{\text {atom }}(t)\right]+\mathcal{D}^{\dagger}[L] c(t)\right| \psi_{0}\right\rangle \mathrm{d} t \\
& =\operatorname{tr}_{\text {atom }}\left[\left(-i\left[c(t), H_{\text {atom }}(t)\right]+\mathcal{D}^{\dagger}[L] c(t)\right) \rho(0)\right]
\end{aligned}
$$

But since we also have

$$
\langle\mathrm{d} c(t)\rangle=\operatorname{tr}_{\text {atom }}[c(0) \mathrm{d} \rho(t)],
$$

the cyclic property of the trace gives us an equation of motion for the effective density matrix,

$$
\mathrm{d} \rho(t)=-i\left[H_{\text {atom }}, \rho(t)\right] \mathrm{d} t+\mathcal{D}[L] \rho(t) \mathrm{d} t,
$$

where we have removed the time arguments $t=0$ from the Schrödinger picture atom operators. Remembering that $L=\sqrt{\Gamma} \sigma_{-}$, we see that we have rederived the master equation for the atom, Eq. (3.13).

\subsection{Fock-state input}

As a first application of quantum stochastic calculus, we can consider a specific type of input to our quantum system (our atom): a Fock state with a specific number of excitations (e.g., photons). This situation is especially important for papers II and V, where we design a photon detector and test it by simulating an input of one or zero photons.

A general formalism for handling Fock-state input was developed very recently $[263,264]$, building on some earlier efforts [265, 266]. Here, we will follow Ref. [263] to derive a generalization of the master equation from Sec. 3.1 which encompasses the fact that $N$ photons are impinging on our quantum system. The setup we consider is schematically depicted in Fig. 3.1. 


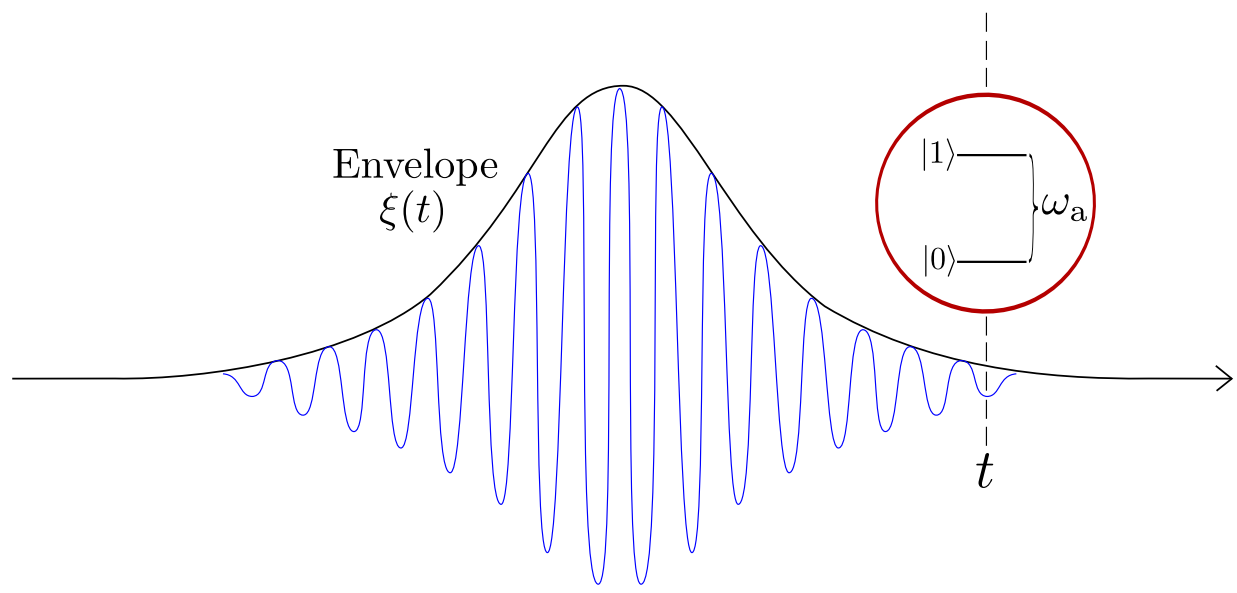

Figure 3.1: A Fock-state wavepacket with envelope $\xi(t)$ interacting with a quantum system. In this illustration, the quantum system is a two-level atom. The time $t$ is defined by when the wavepacket part $\xi(t)$ interacts with the atom.

\subsubsection{Deriving the coupled master equations}

First, we define the Fock state $[267,268]$

$$
\left|1_{\xi}\right\rangle=\int_{-\infty}^{\infty} \mathrm{d} \omega \tilde{\xi}(\omega) b^{\dagger}(\omega)|0\rangle
$$

where $\tilde{\xi}(\omega)$ is the spectral density function describing how the photon is superposed over different modes. The spectrum is assumed to be confined to a small bandwidth around a center frequency $\omega_{c}$, which is close to the relevant transition frequency of the system the photon is going to interact with. Under this assumption, we can then define a slowly varying envelope $\tilde{\xi}(\omega) \rightarrow \tilde{\xi}(\omega) e^{-i \omega_{c} t}$. The Fourier transform of this envelope, $\mathcal{F}[\tilde{\xi}(\omega)]=\xi(t)$, is the temporal shape of the photon, normalized according to $\int \mathrm{d} t|\xi(t)|^{2}=1$. In the time domain we then have

$$
\left|1_{\xi}\right\rangle=\int_{-\infty}^{\infty} \mathrm{d} t \xi(t) b_{\mathrm{in}}^{\dagger}(t)|0\rangle,
$$

and a Fock state with $N$ photons can be defined as

$$
\left|N_{\xi}\right\rangle=\frac{1}{\sqrt{N}}\left(\int_{-\infty}^{\infty} \mathrm{d} t \xi(t) b_{\text {in }}^{\dagger}(t)\right)^{N}|0\rangle .
$$


Assuming, like we did in Sec. 3.3, that the atom+bath state factorizes at time $t=0$ as $|\psi\rangle \otimes|0\rangle$, where $|\psi\rangle$ is some pure atom state, we can now proceed in a similar manner as in that section to derive an equation for the effective density matrix of the atom. It turns out to be more complicated than before, since the atom now can absorb excitations from the bath, which was not the case when the bath was in the vacuum state. Recall that all equations in Sec. 3.3 were derived using the Ito vacuum expectation rules for combinations of $\mathrm{d} B_{t}$ and $\mathrm{d} B_{t}^{\dagger}$. Fortunately, it can be shown that these rules still hold unchanged even for asymmetric Fock state expectation values $\left\langle M_{\xi}|\ldots| N_{\xi}\right\rangle$ [263]. Thus, Eqs. (3.38) and (3.39) can still be used.

Using the identity

$$
\mathrm{d} B_{t}\left|N_{\xi}\right\rangle=\mathrm{d} t \sqrt{N} \xi(t)\left|(N-1)_{\xi}\right\rangle,
$$

which can be shown rather easily [263], together with Eq. (3.39) gives that the equation of motion for the expectation value of an atom operator $c$ in the Heisenberg picture is

$$
\begin{aligned}
\langle\mathrm{d} c(t)\rangle= & \left\langle\psi, N_{\xi}|\mathrm{d} c(t)| \psi, N_{\xi}\right\rangle \\
= & \left\langle\psi, N_{\xi}\left|-i\left[c(t), H_{\text {atom }}(t)\right]+\mathcal{D}^{\dagger}[L(t)] c(t)\right| \psi, N_{\xi}\right\rangle \mathrm{d} t \\
& +\left\langle\psi, N_{\xi}\left|[c(t), L(t)] \mathrm{d} B_{t}^{\dagger}+\left[L^{\dagger}(t), c(t)\right] \mathrm{d} B_{t}\right| \psi, N_{\xi}\right\rangle \\
= & \left\langle\psi, N_{\xi}\left|-i\left[c(t), H_{\text {atom }}(t)\right]+\mathcal{D}^{\dagger}[L(t)] c(t)\right| \psi, N_{\xi}\right\rangle \mathrm{d} t \\
& +\left\langle\psi,(N-1)_{\xi}|[c(t), L(t)]| \psi, N_{\xi}\right\rangle \xi^{*}(t) \mathrm{d} t \\
& +\left\langle\psi, N_{\xi}\left|\left[L^{\dagger}(t), c(t)\right]\right| \psi,(N-1)_{\xi}\right\rangle \xi(t) \mathrm{d} t .
\end{aligned}
$$

Apparently, we need to evaluate several expectation values on the form $\left\langle\psi, n_{\xi}|X(t)| \psi, m_{\xi}\right\rangle$ for different atom operators $X(t)$ in order to eventually find $\langle c(t)\rangle$. From Eqs. (3.39) and (3.49) we get

$$
\begin{aligned}
\left\langle\psi, n_{\xi}|\mathrm{d} X(t)| \psi, m_{\xi}\right\rangle= & \left\langle\psi, n_{\xi}\left|-i\left[X(t), H_{\mathrm{atom}}(t)\right]\right| \psi, m_{\xi}\right\rangle \mathrm{d} t \\
& +\left\langle\psi, n_{\xi}\left|\mathcal{D}^{\dagger}[L(t)] X(t)\right| \psi, m_{\xi}\right\rangle \mathrm{d} t \\
& +\left\langle\psi,(n-1)_{\xi}|[X(t), L(t)]| \psi, m_{\xi}\right\rangle \sqrt{n} \xi^{*}(t) \mathrm{d} t \\
& +\left\langle\psi, n_{\xi}\left|\left[L^{\dagger}(t), X(t)\right]\right| \psi,(m-1)_{\xi}\right\rangle \sqrt{m} \xi(t) \mathrm{d} t .
\end{aligned}
$$

Thus, we see that we end up with a system of equations that couple downwards. Solving these equations starting from $\langle\psi, 0|X(t)| \psi, 0\rangle$ will eventu- 
ally give $\langle c(t)\rangle$. The initial conditions are

$$
\left\langle\psi, n_{\xi}|X(0)| \psi, m_{\xi}\right\rangle= \begin{cases}\langle\psi|X(0)| \psi\rangle, & \text { if } n=m, \\ 0, & \text { if } n \neq m .\end{cases}
$$

To get a less cumbersome description in terms of an effective atom density matrix instead, we define $\rho_{m, n}$ in analogy with Eq. (3.41):

$$
\left\langle\psi, n_{\xi}|X(t)| \psi, m_{\xi}\right\rangle=\operatorname{tr}_{\text {atom }}\left(\rho_{m, n}(t) X\right) .
$$

With this definition Eq. (3.51) can either be written as

$$
\begin{aligned}
\left\langle\psi, n_{\xi}|\mathrm{d} X(t)| \psi, m_{\xi}\right\rangle= & \operatorname{tr}_{\text {atom }}\left(\rho_{m, n}(t)\left(-i\left[X, H_{\text {atom }}\right]+\mathcal{D}^{\dagger}[L] X\right)\right) \mathrm{d} t \\
& +\operatorname{tr}_{\text {atom }}\left(\rho_{m, n-1}(t)[X, L]\right) \sqrt{n} \xi^{*}(t) \mathrm{d} t \\
& +\operatorname{tr}_{\text {atom }}\left(\rho_{m-1, n}(t)\left[L^{\dagger}, X\right]\right) \sqrt{m} \xi(t) \mathrm{d} t, \quad(3.54)
\end{aligned}
$$

or as

$$
\left\langle\psi, n_{\xi}|\mathrm{d} X(t)| \psi, m_{\xi}\right\rangle=\operatorname{tr}_{\text {atom }}\left(\mathrm{d} \rho_{m, n}(t) X\right) .
$$

Combining these two equations and using the cyclic property of the trace gives the system of equations

$$
\begin{aligned}
\frac{\mathrm{d} \rho_{m, n}(t)}{\mathrm{d} t}= & -i\left[H, \rho_{m, n}(t)\right]+\mathcal{D}[L] \rho_{m, n}(t) \\
& +\sqrt{n}\left[L, \rho_{m, n-1}(t)\right] \xi^{*}(t) \\
& +\sqrt{m}\left[\rho_{m-1, n}(t), L^{\dagger}\right] \xi(t),
\end{aligned}
$$

which can be solved by starting from the equation for $\rho_{0,0}(t)$ and working your way upwards. From Eqs. (3.52) and (3.53) it follows that the initial conditions are

$$
\rho_{m, n}(0)= \begin{cases}|\psi\rangle\langle\psi|, & \text { if } n=m, \\ 0, & \text { if } n \neq m .\end{cases}
$$

Note that $\rho_{m, n}=\rho_{n, m}^{\dagger}$, which reduces the number of equations that need to be solved. The final result is $\rho_{N, N}(t)$, which allows us to calculate the expectation value of any atom operator when a wavepacket containing $N$ photons interacts with the atom.

This formalism can be extended in a number of ways. For example, it allows for the bath state to be in a superposition of Fock states. Also, one can include a coupling between the atom and the gauge process increment $\mathrm{d} \Lambda(t)=b_{\mathrm{in}}^{\dagger} b_{\mathrm{in}} \mathrm{d} t$, but these things are outside the scope of this thesis as they are not used in Papers II and V. The interested reader is referred to Refs. [263, 264]. 


\subsubsection{Example - a photon meeting an atom}

To illustrate the formalism for Fock-state input, we will now look more closely at the simplest example possible: a single-photon wavepacket impinging on a two-level atom. The atom Hamiltonian is, in the rotating frame of the photon center frequency $\omega_{\mathrm{ph}}$,

$$
H_{\text {atom }}=\frac{\Delta}{2} \sigma_{z},
$$

where $\Delta=\omega_{\mathrm{a}}-\omega_{\mathrm{ph}}$. We assume the photon wavepacket to have the Gaussian envelope

$$
\xi(t)=\left(\frac{\Gamma_{\mathrm{ph}}^{2}}{2 \pi}\right)^{1 / 4} \exp \left(-\frac{\Gamma_{\mathrm{ph}}^{2}\left(t-t_{\mathrm{ph}}\right)^{2}}{4}\right),
$$

where $t_{\mathrm{ph}}$ is the time when the center of the photon wavepacket arrives at the atom and $\Gamma_{\mathrm{ph}}$ is the bandwidth of the photon.

The coupled density matrix equations become

$$
\begin{aligned}
\dot{\rho}_{0,0}= & -i\left[\frac{\Delta}{2} \sigma_{z}, \rho_{0,0}\right]+\Gamma \mathcal{D}\left[\sigma_{-}\right] \rho_{0,0}, \\
\dot{\rho}_{0,1}= & -i\left[\frac{\Delta}{2} \sigma_{z}, \rho_{0,1}\right]+\Gamma \mathcal{D}\left[\sigma_{-}\right] \rho_{0,1}+\xi^{*}(t)\left[\sqrt{\Gamma} \sigma_{-}, \rho_{0,0}\right], \\
\rho_{1,0}= & \rho_{0,1}^{\dagger} \\
\dot{\rho}_{1,1}= & -i\left[\frac{\Delta}{2} \sigma_{z}, \rho_{1,1}\right]+\Gamma \mathcal{D}\left[\sigma_{-}\right] \rho_{1,1}+\xi(t)\left[\rho_{0,1}, \sqrt{\Gamma} \sigma_{+}\right] \\
& +\xi^{*}(t)\left[\sqrt{\Gamma} \sigma_{-}, \rho_{1,0}\right]
\end{aligned}
$$

with initial conditions $\rho_{0,0}(0)=\rho_{1,1}(0)=\rho_{\text {atom }}(0)$, and $\rho_{0,1}(0)=\rho_{1,0}(0)=$ 0 .

The density matrix can be solved analytically by writing $\rho_{m, n}$ as a combination of Pauli matrices [269]. However, as this is somewhat tedious, we limit ourselves to plotting, in Fig. 3.2, numerical solutions for the atom excitation probability $P_{\text {exc }}(t)$. This shows how the arrival of the photon leads to a high probability of exciting the atom when the photon is on resonance. The exact probability depends on the wavepacket shape [235].

In paper II, we perform a similar calculation to model the effect of a single photon arriving at a three-level transmon. The photon frequency is close to the first transition frequency of the transmon. We study, for 


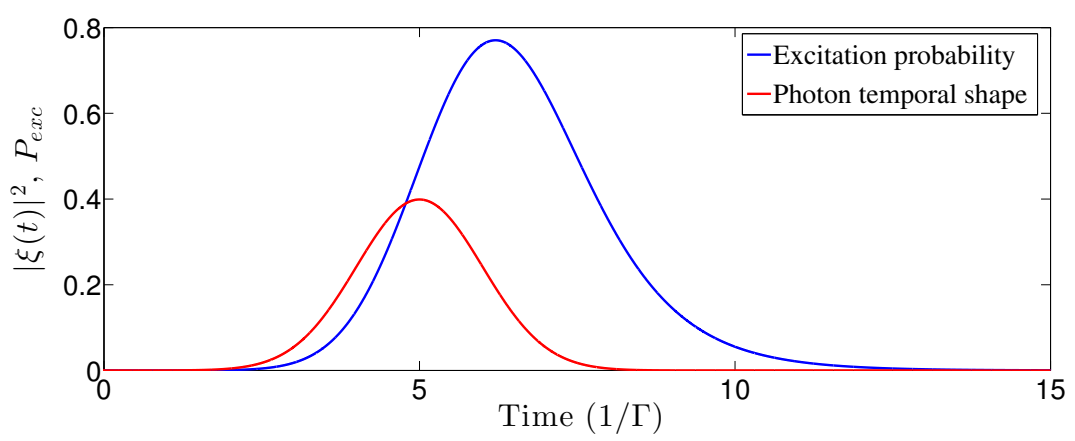

Figure 3.2: The dynamics of a single-photon wavepacket encountering a two-level atom. The red line shows the temporal shape of the Gaussian wavepacket of the photon, and the blue line shows the excitation probability of the atom as a function of time. Parameters: $\Delta=0, t_{\mathrm{ph}}=5 / \Gamma, \Gamma_{\mathrm{ph}}=\Gamma, \rho_{\text {atom }}(0)=|g\rangle\langle g|$.

different shapes of the photon wavepacket, how the presence of the photon affects a coherent probe which is close in frequency to the second transition of the transmon. Even in this case, one can get far by a purely analytical treatment. However, in Paper V we include several cascaded transmons; the Fock-state input formalism is still used, but we are restricted to numerical simulations.

In this chapter, we have developed formalisms to handle open quantum systems. We can now give an effective description for how a quantum system dissipates excitations to an environment and how input-output works for such a system. In the next chapter, we will use the quantum stochastic calculus further to answer the question: what is the effect of a measurement on the output from the quantum system? 



\section{Chapter 4}

\section{Quantum measurements}

To characterize a quantum system, we need to measure it. In this way, measurements are an integral part of all the appended papers that include experiments (III, IV, VI, and VIII). However, the purely theoretical Papers I, II, and V are also concerned with measurements, or rather how to improve them. It transpires that measuring a quantum system is more complicated than measuring a classical one. This is due to two things: unavoidable noise from quantum vacuum fluctuations and measurement back-action, i.e., measuring one component of an entangled state can alter the other components, as was discussed in Sec. 1.4.

In this chapter, we will first look at an example to illustrate further how a measurement on the output from a quantum system can give rise to back-action on that system. We will then use the stochastic quantum calculus from Chapter 3 to derive stochastic master equations (SMEs) describing the effect of both photon detection and homodyne detection on the system output. Finally, we consider another type of measurement, used in experiments in some of the appended papers, where both drive and probe tones are applied to the system. This two-tone spectroscopy is most easily understood by calculating the susceptibility of the system.

\subsection{Measurement back-action and SMEs}

Our goal of this section is to incorporate the effect of measurements in the time evolution of the system density matrix. We will begin with a simple example to gain more intuition into the effects of a measurement. 


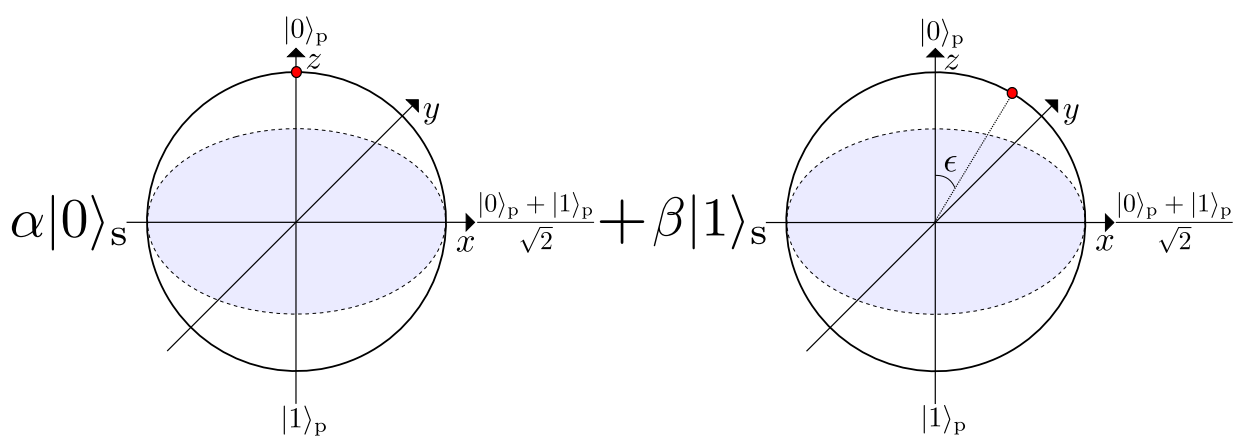

Figure 4.1: The weakly entangled state of Eq. (4.3) illustrated using the Bloch sphere representation for the probe qubit.

\subsubsection{An example of back-action}

Imagine that we have a system consisting of a qubit in the superposition state

$$
|\Psi\rangle_{\mathrm{s}}=\alpha|0\rangle+\beta|1\rangle
$$

We now want to do a measurement on this system. A measurement will involve sending in a probe to interact with the system. We will use the simplest probe possible, a second qubit. This probe qubit interacts with the system qubit for some time, entangling the two, and then enters a measurement apparatus which can do a projective measurement. From the result of the projective measurement on the probe qubit, we will try to infer information about the system qubit state.

The simplest version of this protocol is when the two qubits become fully entangled, giving a state for system + probe

$$
|\Psi\rangle_{\mathrm{s}+\mathrm{p}}=\alpha|00\rangle+\beta|11\rangle .
$$

In this case, measuring the probe qubit to be in state $|\Psi\rangle_{\mathrm{p}}=|1\rangle(|0\rangle)$ will then project the system qubit into the state $|\Psi\rangle_{\mathrm{s}}=|1\rangle(|0\rangle)$. In other words, the act of measuring the probe qubit gives back-action on the system qubit.

There are two ways we can alter the protocol above to get more interesting back-action. Firstly, we can choose to measure the probe qubit in some other basis (above we measured in the $Z$ basis). Secondly, the entanglement can be weaker. Consider the weakly entangled state

$$
|\Psi\rangle_{\mathrm{s}+\mathrm{p}}=\alpha|00\rangle+\beta|1\rangle_{\mathrm{s}}\left(\cos \frac{\epsilon}{2}|0\rangle_{\mathrm{p}}+\sin \frac{\epsilon}{2}|1\rangle_{\mathrm{p}}\right),
$$


pictured in Fig. 4.1. If we now measure the probe qubit in the $Z$ basis we get

$$
|\Psi\rangle_{\mathrm{s}}= \begin{cases}\frac{\alpha|0\rangle+\beta \cos \epsilon / 2|1\rangle}{\sqrt{|\alpha|^{2}+|\beta|^{2} \cos ^{2} \epsilon / 2}}, & \text { if } Z=+1 \\ |1\rangle, & \text { if } Z=-1\end{cases}
$$

That is, measuring $Z=+1$, which we do with probability $|\alpha|^{2}+|\beta|^{2} \cos ^{2} \epsilon / 2$, only gives us a little information about the system qubit if $\epsilon$ is small. In turn, the back-action of the measurement is small. If we look at the probe qubit in the $Y$ basis instead we find

$$
|\Psi\rangle_{\mathrm{s}}= \begin{cases}\alpha|0\rangle+\beta e^{i \epsilon / 2}|1\rangle, & \text { if } Y=+1 \\ \alpha|0\rangle+\beta e^{-i \epsilon / 2}|1\rangle, & \text { if } Y=-1\end{cases}
$$

Here, we see that the measurement does not give any information about the probabilities of finding the system qubit in state $|0\rangle$ or $|1\rangle$. This is because the probabilities for the different $Y$ measurement results are always $1 / 2$ each; they do not depend on $\alpha$ or $\beta$. However, the $Y$ measurement still gives rise to a back-action on the system qubit in the form of a phase kick. Moreover, provided that we know the degree of entanglement $\epsilon$, we know exactly what the phase kick was given the measurement result.

This last example is closely connected to the work in Paper I. There, we consider sending in probe photons which become entangled with a system qubit. Measuring the photons in one way gives information about the qubit state, but measuring them in another way only gives rise to a measurement back-action in the form of phase kicks. These phase kicks can be calculated and undone given the measurement record, which becomes important if the system is scaled up to two qubits and one wants to do a parity measurement on them.

\subsubsection{Time evolution of the density matrix}

In the example above, we arrived at the final state of the system, conditioned on the measurement result, by projecting into the subspace corresponding to the measurement result and then tracing out the probe. In general, we consider the time evolution of a density matrix

$$
\rho(t)=U(t) \rho(0) U^{\dagger}(t)
$$

for the combined Hilbert space of the system and the probe. We use the abbreviated notation $U(t)=U(t, 0)$ for the time evolution operator. 
The measurement can give different outcomes $i$, corresponding to projecting the probe into the state $|i\rangle_{\mathrm{p}}$ in the Hilbert space of the probe. The probability of outcome $i$ is $p_{i}=\operatorname{tr}(|i\rangle\langle i|\rho| i\rangle\langle i|)$. If we assume that the probe is initialized in some ground state $|0\rangle_{p}$, then the system density matrix, after the measurement result becomes $i$, is given by

$$
\rho_{i}^{\mathrm{s}}(t)=\frac{1}{p_{i}}\left\langle i\left|U(t)\left(\rho^{\mathrm{s}}(0) \otimes|0\rangle\langle 0|\right) U^{\dagger}(t)\right| i\right\rangle=\frac{1}{p_{i}} \Omega_{i}(t) \rho^{\mathrm{s}}(0) \Omega_{i}^{\dagger}(t),
$$

where we defined the operator $\Omega_{i}(t)=\langle i|U(t)| 0\rangle$. This operator lives in the system Hilbert space and gives an effective description of the time evolution of the system conditioned on measurement results. Note that the probability can now be rewritten $p_{i}(t)=\operatorname{tr}\left(\Omega_{i}(t) \rho^{\mathrm{s}}(0) \Omega_{i}^{\dagger}(t)\right)$.

Assuming that the measurement takes place during some short time $\mathrm{d} t$, we arrive at the equation of motion for the system density matrix [122, $177]$,

$$
\rho_{i}(t+\mathrm{d} t)=\frac{1}{p_{i}(\mathrm{~d} t)} \Omega_{i}(\mathrm{~d} t) \rho(t) \Omega_{i}^{\dagger}(\mathrm{d} t),
$$

where we have dropped the superscript on $\rho$. It is important to note that Eq. (4.8) is very different from the master equation we derived in Sec. 3.1. The difference is that we have now allowed for measurements, which means that probabilities come into play and turns Eq. (4.8) into a stochastic equation. An equation of this type, which gives the time evolution of a system conditioned on measurement results, is often referred to as a quantum trajectory equation [270-273]. While an ordinary master equation describes the average evolution of a system over many experiments, a quantum trajectory is like a single run of an experiment. Thus, averaging over the possible measurement outcomes (many runs of the experiment) will let us recover the ordinary master equation from a quantum trajectory equation; we will see examples of this in the following sections.

\subsection{Photon detection}

As a first example of a quantum measurement, we will discuss photon detection. To do this, we consider our quantum system to be a harmonic oscillator with annihilation operator $a$ such that $H_{\mathrm{sys}}=\omega_{\mathrm{r}} a^{\dagger} a$. This system is coupled to a bath of harmonic oscillators just like the atom in the examples of the previous chapter. Given that the relaxation rate of our system is $\kappa$, we have $L=\sqrt{\kappa} a$. 
To begin with, we assume that all photons leaking out from the resonator arrives at the detector. Two measurement outcomes are possible during a short time $\mathrm{d} t$ : either no photon is detected or one photon is detected. We can calculate the corresponding $\Omega_{i}$ operators using the expression for the time evolution operator from Eq. (3.38), modified to a harmonic oscillator:

$$
\begin{aligned}
\Omega_{0}(t+\mathrm{d} t, t)= & \left\langle 0\left|1-i H_{\mathrm{sys}} \mathrm{d} t+\sqrt{\kappa}\left(a \mathrm{~d} B_{t}^{\dagger}-a^{\dagger} \mathrm{d} B_{t}\right)-\frac{\kappa}{2} a^{\dagger} a \mathrm{~d} t\right| 0\right\rangle \\
= & 1-i H_{\text {sys }} \mathrm{d} t-\frac{\kappa}{2} a^{\dagger} a \mathrm{~d} t \\
\Omega_{1}(t+\mathrm{d} t, t)= & \left\langle 1\left|1-i H_{\mathrm{sys}} \mathrm{d} t+\sqrt{\kappa}\left(a \mathrm{~d} B_{t}^{\dagger}-a^{\dagger} \mathrm{d} B_{t}\right)\right| 0\right\rangle \\
& +\left\langle 1\left|\left(-i H_{\mathrm{sys}} \mathrm{d} t+\sqrt{\kappa}\left(a \mathrm{~d} B_{t}^{\dagger}-a^{\dagger} \mathrm{d} B_{t}\right)\right)^{2}\right| 0\right\rangle \\
= & \sqrt{\kappa} a \sqrt{\mathrm{d} t}
\end{aligned}
$$

where we have used the Itō rules for vacuum expectation values and $|1\rangle=$ $\frac{1}{\sqrt{d t}} \mathrm{~d} B^{\dagger}(t)|0\rangle[272]$.

Inserting these results in Eq. (4.8) gives

$$
\begin{aligned}
\rho_{0}(t+\mathrm{d} t)= & \frac{\left(1-i H_{\mathrm{sys}} \mathrm{d} t-\frac{\kappa}{2} a^{\dagger} a \mathrm{~d} t\right) \rho_{0}(t)\left(1+i H_{\mathrm{sys}} \mathrm{d} t-\frac{\kappa}{2} a^{\dagger} a \mathrm{~d} t\right)}{\left\langle\left(1+i H_{\mathrm{sys}} \mathrm{d} t-\frac{\kappa}{2} a^{\dagger} a \mathrm{~d} t\right)\left(1-i H_{\mathrm{sys}} \mathrm{d} t-\frac{\kappa}{2} a^{\dagger} a \mathrm{~d} t\right)\right\rangle} \\
= & \frac{\rho_{0}(t)-i\left[H_{\mathrm{sys}}, \rho_{0}(t)\right] \mathrm{d} t-\frac{\kappa}{2}\left\{a^{\dagger} a, \rho_{0}(t)\right\} \mathrm{d} t+\mathcal{O}\left(\mathrm{d} t^{2}\right)}{1-\kappa\left\langle a^{\dagger} a\right\rangle \mathrm{d} t+\mathcal{O}\left(\mathrm{d} t^{2}\right)} \\
= & \left(\rho_{0}(t)-i\left[H_{\mathrm{sys}}, \rho_{0}(t)\right] \mathrm{d} t-\frac{\kappa}{2}\left\{a^{\dagger} a, \rho_{0}(t)\right\} \mathrm{d} t\right) \\
& \times\left(1+\kappa\left\langle a^{\dagger} a\right\rangle \mathrm{d} t\right)+\mathcal{O}\left(\mathrm{d} t^{2}\right) \\
= & \rho_{0}(t)-i\left[H_{\mathrm{sys}}, \rho_{0}(t)\right] \mathrm{d} t-\frac{\kappa}{2}\left\{a^{\dagger} a, \rho_{0}(t)\right\} \mathrm{d} t \\
& +\kappa\left\langle a^{\dagger} a\right\rangle \rho_{0}(t) \mathrm{d} t+\mathcal{O}\left(\mathrm{d} t^{2}\right)
\end{aligned}
$$

and carrying out a similar calculation for $\rho_{1}(t)$ leaves us with

$$
\begin{aligned}
\mathrm{d} \rho_{0} & =\left(-i\left[H_{\text {sys }}, \rho_{0}\right]+\kappa\left\langle a^{\dagger} a\right\rangle \rho_{0}-\frac{\kappa}{2}\left\{a^{\dagger} a, \rho_{0}\right\}\right) \mathrm{d} t, \\
\mathrm{~d} \rho_{1} & =\frac{a \rho_{1} a^{\dagger}}{\left\langle a^{\dagger} a\right\rangle}-\rho_{1},
\end{aligned}
$$


where we suppressed the time arguments for brevity.

To combine these results into a single SME, we define the stochastic process $N(t)$, which counts the number of photons detected up to time $t$. We then have the stochastic increment $\mathrm{d} N(t)$ with the property $\mathrm{d} N(t)^{2}=\mathrm{d} N(t)$, since in a small enough time interval one can only detect 0 or 1 photons. The stochastic increment also has the expectation value $\mathrm{E}[\mathrm{d} N(t)]=\kappa\left\langle a^{\dagger} a\right\rangle \mathrm{d} t$, which is the probability of detecting one photon during the time $\mathrm{d} t$. With this notation, Eqs. (4.12) and (4.13) give the SME

$$
\begin{aligned}
\mathrm{d} \rho= & \left(-i\left[H_{\mathrm{sys}}, \rho\right]+\kappa\left\langle a^{\dagger} a\right\rangle \rho-\frac{\kappa}{2}\left\{a^{\dagger} a, \rho\right\}\right) \mathrm{d} t \\
& +\left(\frac{a \rho a^{\dagger}}{\left\langle a^{\dagger} a\right\rangle}-\rho\right) \mathrm{d} N(t),
\end{aligned}
$$

which describes how the system state develops, conditioned on the measurement record $N(t)$. To make the connection to the master equations of Sec. 3.1, remember that Eq. (4.14) is a quantum trajectory equation. If we average over many trajectories, using the expectation value for $\mathrm{d} N(t)$, we recover a master equation on the Lindblad form,

$$
\dot{\rho}=-i\left[H_{\mathrm{sys}}, \rho\right]+\kappa \mathcal{D}[a] \rho .
$$

In the above derivation, we assumed that all output from the system reaches the photon detector, and that the detector is perfect. A more realistic situation is one where the detector only registers a fraction $\eta$ of the photons from the system. The parameter $\eta$ is called the measurement efficiency. When $\eta<1$, the SME will include an ordinary Lindblad term describing the loss of the undetected photons. This clarifies the picture of the ordinary master equation as a description of average effects on the system where we lack information of their exact behaviour in each trajectory. The final expression for the SME including measurement efficiency is [272]

$$
\begin{aligned}
\mathrm{d} \rho= & \left(-\frac{i}{\hbar}\left[H_{\text {sys }}, \rho\right]+(1-\eta) \kappa \mathcal{D}[a] \rho\right) \mathrm{d} t \\
& +\mathcal{G}[a] \rho \mathrm{d} N(t)-\frac{1}{2} \eta \kappa \mathcal{M}\left[a^{\dagger} a\right] \rho \mathrm{d} t,
\end{aligned}
$$

where we have introduced the notation $\mathcal{G}[c] \rho=\frac{c \rho c^{\dagger}}{\left\langle c^{\dagger} c\right\rangle}-\rho, \mathcal{M}[c] \rho=c \rho+$ $\rho c^{\dagger}-\left\langle c+c^{\dagger}\right\rangle \rho$, and where now $\mathrm{E}[\mathrm{d} N(t)]=\eta \kappa\left\langle a^{\dagger} a\right\rangle \mathrm{d} t$. An SME of this 
type, with the addition of a qubit coupled to the harmonic oscillator, is central to Paper I, where we among other things investigate how to undo unwanted measurement back-action from a photon detection measurement.

In Sec. 1.1, we related how an efficient single-photon detector for propagating photons is missing in the experimental toolbox of circuit QED, and how Papers II and V are concerned with fixing this problem. However, the proposed setup in those papers is not directly described by an SME like Eq. (4.16), but instead uses a homodyne measurement on a coherent probe signal to realize an effective photon detection. The theoretical description of such a measurement is the topic of the following section.

\subsection{Homodyne detection}

In the absence of a good photon detector, homodyne detection is the chief measurement technique used in quantum optics with superconducting circuits. In this section, we will derive an SME for homodyne detection following Refs. [177, 254]. A different derivation can be found in Ref. [272].

A simple theoretical model of homodyne detection is depicted in Fig. 4.2. The output from the system that we wish to measure is fed into one of the two input ports of a 50/50 beamsplitter. At the other input port, a strong coherent signal at the same frequency from a local oscillator (LO), is applied. The two outputs from the beamsplitter are then detected by separate photon detectors and the final measurement signal, the homodyne current, is the difference of the two photocurrents. It should be clearly noted that this is a theoretical model which captures the essential physics. The actual experimental implementation for superconducting circuits is different; since microwave photon detectors are lacking, the signal is first amplified, then down-converted in frequency, and finally sampled with an analog-to-digital converter [274, 275].

We can use the same formalism as in the previous section to derive the $\Omega_{i}$ operators for each of the two photon detectors. However, we must remember that the inputs to the two detectors are mixes of $\mathrm{d} B_{t}$, driven by the coherent signal, and $\mathrm{d} A_{t}$, driven by the system output. For a $50 / 50$ beamsplitter, these mixes are given by

$$
\begin{aligned}
\mathrm{d} C_{t}^{(1)} & =\frac{1}{\sqrt{2}}\left(\mathrm{~d} A_{t}+\mathrm{d} B_{t}\right), \\
\mathrm{d} C_{t}^{(2)} & =\frac{1}{\sqrt{2}}\left(\mathrm{~d} A_{t}-\mathrm{d} B_{t}\right) .
\end{aligned}
$$




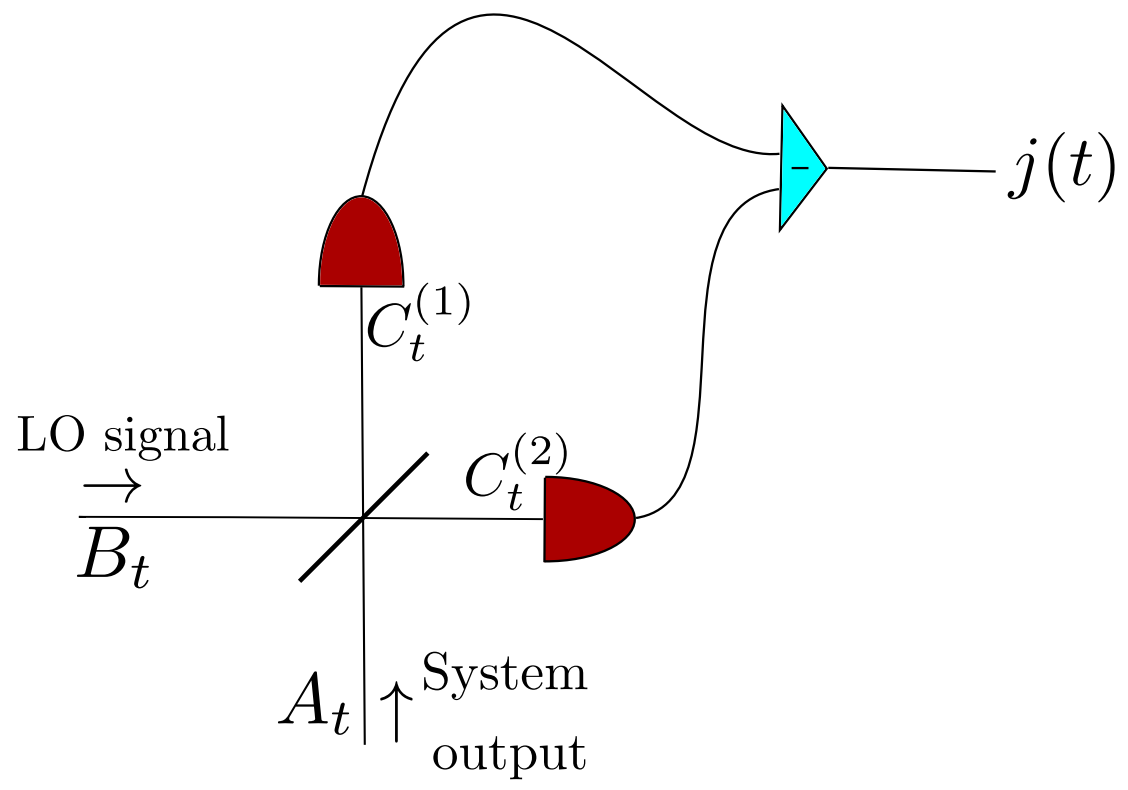

Figure 4.2: The model for homodyne detection. The system output is mixed with a strong coherent signal at a beamsplitter and photon detection (red devices in the figure) is performed on the two outputs. The homodyne current $j(t)$ is found as the difference of the two photon detector signals in the limit of an infinitely strong local oscillator.

We must also find the time evolution operator for the $B$ vacuum. The coherent signal from the LO, with amplitude $\beta\left(|\beta|^{2}\right.$ is the photon flux), gives terms like $\beta^{*} \mathrm{~d} B_{t}$ instead of $\sqrt{\kappa} a^{\dagger} \mathrm{d} B_{t}$ as we had previously. Keeping in mind that $|0\rangle$ now really means $|0\rangle_{A} \otimes|0\rangle_{B}$, we get

$$
\begin{aligned}
\Omega_{0}(t+\mathrm{d} t, t)= & \left\langle 0\left|1-i H_{\mathrm{sys}} \mathrm{d} t+\sqrt{\kappa}\left(a \mathrm{~d} A_{t}^{\dagger}-a^{\dagger} \mathrm{d} A_{t}\right)+\beta \mathrm{d} B_{t}^{\dagger}\right| 0\right\rangle \\
& +\left\langle\left. 0\left|-\beta^{*} \mathrm{~d} B_{t}-\frac{\kappa}{2} a^{\dagger} a \mathrm{~d} t-\right| \beta\right|^{2} \mathrm{~d} t \mid 0\right\rangle \\
= & 1-i H_{\mathrm{sys}} \mathrm{d} t-\frac{\kappa}{2}\left(a^{\dagger} a+|\beta|^{2}\right) \mathrm{d} t \\
\Omega_{1 / 2}(t+\mathrm{d} t, t)= & \sqrt{\frac{\kappa}{2}}(a \pm \beta) \sqrt{\mathrm{d} t}
\end{aligned}
$$

where we used $\left\langle\left. 1\right|_{1 / 2}=\frac{1}{\sqrt{2} \sqrt{\mathrm{d} t}}\langle 0|\left(\mathrm{d} A_{t} \pm \mathrm{d} B_{t}\right)\right.$ and the Itō rules. With these results in hand, we can write down the equation of motion for the density matrix for the three different measurement results. Defining the stochas- 
tic photon counting processes $N_{1}(t)$ and $N_{2}(t)$ with their corresponding stochastic increments like in the previous section, we get an SME.

The SME for homodyne detection then follows as a result of taking the limit of an infinitely strong LO, i.e., $\beta \rightarrow \infty$. Carrying through this analysis, which requires some work that is well described elsewhere [177, 254], eventually leads to the SME

$$
\mathrm{d} \rho=-i\left[H_{\mathrm{sys}}, \rho\right] \mathrm{d} t+\kappa \mathcal{D}[a] \rho \mathrm{d} t+\sqrt{\kappa \eta} \mathcal{M}\left[a e^{-i \phi}\right] \rho \mathrm{d} W(t),
$$

where $\mathrm{d} W(t)$ is a Wiener increment, $\phi$ is a phase set by the LO, and we have included the measurement efficiency $\eta$. The phase $\phi$ determines which quadrature of the signal is measured. The Wiener increment is a random variable with $\mathrm{E}[\mathrm{d} W(t)]=0$ and variance $\mathrm{d} t$. Using the first property, we see that averaging over many quantum trajectories given by Eq. (4.21) once again lets us recover the ordinary Lindblad master equation, Eq. (4.15).

The measurement signal associated with our SME is the homodyne current

$$
j(t) \mathrm{d} t=\sqrt{\kappa \eta}\left\langle a e^{-i \phi}+a^{\dagger} e^{i \phi}\right\rangle \mathrm{d} t+\mathrm{d} W(t),
$$

which we get by taking the limit $\beta \rightarrow \infty$ of the normalized photocurrent $\left(N_{1}(t)-N_{2}(t)\right) / \beta[177,254]$. Eq. (4.22) shows two things clearly. Firstly, changing $\phi$ indeed determines which quadrature is measured; if $\phi=0$, we get information about $\left\langle a+a^{\dagger}\right\rangle$, while if $\phi=\pi / 2$, we get information about $i\left\langle a^{\dagger}-a\right\rangle$. Secondly, the signal will be noisy, even for a vacuum bath, due to the stochastic increment $\mathrm{d} W(t)$. The latter can be seen as an effect of vacuum fluctuations.

The SME for homodyne detection is important for the calculations in Paper I. There, we study homodyne measurements on a resonator dispersively coupled to one or two qubits. Depending on which quadrature is measured, the information about the qubit state(s) that can be extracted, and consequently the back-action of the measurement on the qubit(s), can be very different. Interestingly, even though the measurement back-action is partly random due to the stochastic increments $\mathrm{d} W(t)$ in Eq. (4.21), we can still extract it exactly from the measured homodyne current, since that also includes $\mathrm{d} W(t)$.

In Papers II and V, we use homodyne detection to construct an effective photon detector. There, we also combine Eq. (4.21) with the formalism for Fock-state input given in Sec. 3.4 and with the formalism for cascaded quantum systems which is the topic of Chapter 5 . The essential question in 
Papers II and V is whether we can get a strong enough signal to overcome the ever-present noise $\mathrm{d} W(t)$.

To demonstrate the effects predicted in Papers I, II, and V in experiment, a high measurement efficiency $\eta$ is essential. Achieving this is not easy; amplifiers are needed to process the weak signals considered, and they generally add a lot more noise on top of that of the quantum vacuum, giving low $\eta$. Fortunately, in the last years parametric amplifiers based on Josephson junctions have been developed, pushing $\eta$ up towards 0.5 and above [276-282].

As a final remark, it should be noted that the SMEs for photon detection and homodyne detection require some extra care when used in analytical or numerical calculations. They are not ordinary differential equations; the stochastic components make them fundamentally different. For general analytical solutions and efficient numerical algorithms, the interested reader can consult Ref. [283].

\subsection{Susceptibility}

In the previous sections, we considered different ways of doing measurements on the output from a quantum system. Another way to characterize the system, often used in experiments, is to investigate its response to one or more external drive signals. To this end, the input-output theory of Sec. 3.2 is useful. However, in two-tone spectroscopy, where the system is driven at one frequency $\omega_{\mathrm{d}}$ and the response to a weak probe at another frequency $\omega_{\mathrm{p}}$ is measured, it becomes hard to find an appropriate rotating frame to work in. In this case, it is helpful to calculate the susceptibility of the system instead. The method is applied in Papers III, IV, and VI.

We follow Ref. [284] and consider a system with Hamiltonian

$$
H_{\text {sys }}=H_{0}+H_{1},
$$

where $H_{1}$ is a small perturbation, caused by a coherent probe signal $\alpha(t)$ being applied to the system operator $c$,

$$
H_{1}=c \alpha(t)=c\left(\alpha e^{-i \omega_{\mathrm{p}} t}+\alpha^{*} e^{i \omega_{\mathrm{p}} t}\right) .
$$

We want to find the response (the change) $\Delta\langle d\rangle$ of a system operator $d$ to this perturbation. Since the perturbation is small, the response can be assumed linear,

$$
\Delta\langle d(t)\rangle=\int_{-\infty}^{t} \mathrm{~d} t^{\prime} \chi_{d c}\left(t-t^{\prime}\right) \alpha\left(t^{\prime}\right),
$$


where $\chi_{d c}(t)$ is the susceptibility.

The time evolution for the system density matrix $\rho=\rho_{0}+\rho_{1}\left(\rho_{1} \ll \rho_{0}\right.$ since the perturbation is weak) is governed by the Liouville-von Neumann equation, Eq. (3.3),

$\dot{\rho}(t)=\dot{\rho}_{0}(t)+\dot{\rho}_{1}(t)=-i\left[H_{0}, \rho_{0}(t)\right]-i\left[H_{0}, \rho_{1}(t)\right]-i\left[H_{1}, \rho_{0}(t)\right]-i\left[H_{1}, \rho_{1}(t)\right]$.

Moving to the interaction picture,

$$
\tilde{\rho}(t)=e^{i H_{0} t} \rho(t) e^{-i H_{0} t},
$$

and ignoring the last term of Eq. (4.26) since it is second order in the perturbation, we get

$$
\dot{\tilde{\rho}}_{1}(t)=-i\left[\tilde{H}_{1}(t), \tilde{\rho}_{0}(t)\right]
$$

which has the solution

$$
\tilde{\rho}_{1}(t)=-i \int_{-\infty}^{t} \mathrm{~d} t^{\prime}\left[\tilde{c}\left(t^{\prime}\right), \tilde{\rho}_{0}\left(t^{\prime}\right)\right] \alpha\left(t^{\prime}\right) .
$$

The response we seek is thus

$$
\Delta\langle d(t)\rangle=\operatorname{tr}\left(\tilde{d}(t) \tilde{\rho}_{1}(t)\right)=i \int_{-\infty}^{t} \mathrm{~d} t^{\prime}\left\langle\left[\tilde{c}\left(t^{\prime}\right), \tilde{d}(t)\right]\right\rangle \alpha\left(t^{\prime}\right),
$$

where the expectation value is taken with respect to the unperturbed density matrix $\rho_{0}$ and we have used that the trace is unchanged under cyclic permutations. We assume $\rho_{0}$ to describe equilibrium, such that the expectation value in Eq. (4.30) only depends on the time difference $t-t^{\prime}$. Making the change of variables $\tau=t-t^{\prime}$, we then have

$$
\Delta\langle d(t)\rangle=i \int_{0}^{\infty} \mathrm{d} \tau\langle[\tilde{c}(0), \tilde{d}(\tau)]\rangle \alpha(\tau-t) .
$$

Fourier transforming this gives

$$
\chi_{d c}\left(\omega_{\mathrm{p}}\right)=i \int_{0}^{\infty} \mathrm{d} \tau\langle[c(0), d(\tau)]\rangle e^{-i \omega_{\mathrm{p}} \tau} .
$$

When we have an open quantum system such that $\rho_{0}$ is governed by a master equation $\dot{\rho}_{0}=\mathcal{L} \rho_{0}$ (where $\mathcal{L}$ is called the Liouvillian and is used 
to abbreviate equations like Eq. (3.13)), the two-time correlators are calculated as [116]

$$
\begin{aligned}
& \langle c(0) d(t)\rangle=\operatorname{tr}\left(d e^{\mathcal{L} t} \rho c\right), \\
& \langle d(t) c(0)\rangle=\operatorname{tr}\left(d e^{\mathcal{L} t} c \rho\right) .
\end{aligned}
$$

For some advice on computing the susceptibility numerically, see Ref. [285].

In this chapter and the previous one, we have now covered master equations for open quantum systems, how to handle input and output in such systems, and how to describe different kinds of measurements on the system output. What remains to discuss is how to handle the output from one system being used as an input for a second one (or fed back as an input for the first). This is the topic of the next chapter. 


\section{Chapter 5}

\section{Cascaded quantum systems}

With artificial atoms enabling quantum optics experiments in more complex, interconnected systems, it becomes important to have theoretical tools that can deal with such setups. In the previous chapters, we have dealt with input-output theory for open quantum systems, developing along the way quantum stochastic calculus which also helped us describe measurements on the system output. In this chapter, we will extend these methods to cascaded quantum systems, where the output from one quantum system is used as the input for another.

Cascaded quantum systems have been studied theoretically for decades $[116,270,286]$, but it is only recently that a compact formalism has been developed which allows for simple calculation of an effective description for any number of cascaded systems. In the following, we will derive the basics of this $(S, L, H)$ formalism [287, 288] and give examples of how it can be applied in situations that arise in some of the appended papers.

\subsection{The $(S, L, H)$ formalism}

Consider the situation depicted in Fig. 5.1, where we have two quantum systems that are described by Hamiltonians $H_{1}$ and $H_{2}$, respectively. We let the first system be coupled via an input-output port to the environment by a coupling operator $L_{1}$. The output from the first system is fed into the second system, which is coupled to the environment via $L_{2}$. The time evolution operator for the combined system can then be found by first time evolving the state of system 1 for a small time $\mathrm{d} t$, then evolving the state of system 2 for that small time, and so on. We assume here that there 


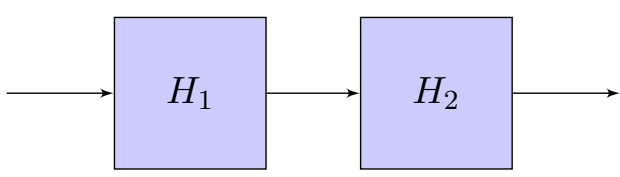

Figure 5.1: Two quantum systems cascaded such that the output from system 1 becomes input to system 2 . Using stochastic quantum calculus, we can derive an effective description for the total system.

is negligible time delay in relaying a signal from the first system to the second. The first part of the time evolution (from time 0 to time $\mathrm{d} t$ ) is then, using Eq. (3.38),

$$
\begin{aligned}
U_{\mathrm{d} t}^{(2)} U_{\mathrm{d} t}^{(1)}= & \left(1+\mathrm{d} U_{0}^{(2)}\right)\left(1+\mathrm{d} U_{0}^{(1)}\right)=1-i\left(H_{1}+H_{2}\right) \mathrm{d} t \\
& +\left(L_{1}+L_{2}\right) \mathrm{d} B_{0}^{\dagger}-\left(L_{1}^{\dagger}+L_{2}^{\dagger}\right) \mathrm{d} B_{0} \\
& -\frac{1}{2}\left(L_{1}^{\dagger} L_{1}+L_{2}^{\dagger} L_{2}\right) \mathrm{d} t-L_{2}^{\dagger} L_{1} \mathrm{~d} t \\
= & 1-i\left(H_{1}+H_{2}+\frac{1}{2 i}\left(L_{2}^{\dagger} L_{1}-L_{1}^{\dagger} L_{2}\right)\right) \mathrm{d} t \\
& -\frac{1}{2}\left(L_{1}+L_{2}\right)^{\dagger}\left(L_{1}+L_{2}\right) \mathrm{d} t \\
& +\left(L_{1}+L_{2}\right) \mathrm{d} B_{0}^{\dagger}-\left(L_{1}+L_{2}\right)^{\dagger} \mathrm{d} B_{0} .
\end{aligned}
$$

From this, we see that the total system behaves as if it had a Hamiltonian $H=H_{1}+H_{2}+\frac{1}{2 i}\left(L_{2}^{\dagger} L_{1}-L_{1}^{\dagger} L_{2}\right)$ and was coupled to the environment via an operator $L=L_{1}+L_{2}[262]$.

The above derivation suggests that an open quantum system could be assigned a doublet $G=(L, H)$, and that the series product of two systems is given by

$$
G=G_{2} \triangleleft G_{1}=\left(L_{1}+L_{2}, H_{1}+H_{2}+\frac{1}{2 i}\left[L_{2}^{\dagger} L_{1}-L_{1}^{\dagger} L_{2}\right]\right) .
$$

Note that the total doublet is not invariant under interchange of 1 and 2. This reflects the ordering of the two systems; the output from one is fed into the other, not the other way around. It is possible to extend the formalism to systems having several input-ouput ports. The $L$ then becomes a column vector of coupling operators and the above expression still holds. 
So far, we have only used the $L$ and $H$ in $(S, L, H)$. The final part of the triplet, $S$, is called the scattering matrix. It is an addition to the formalism needed to describe systems with scattering between multiple channels [288], such as beamsplitters or circulators. Such devices have neither $L$ nor $H$; they are simply systems which take several inputs and mix them into several outputs. The $S$ describes this scattering process, and provides a way to use the $(S, L, H)$ formalism to handle connections between a multitude of different quantum systems. To exemplify, the triplet for a $50 / 50$ beamsplitter is given by

$$
G_{\mathrm{BS}}=\left(\left(\begin{array}{cc}
\frac{1}{\sqrt{2}} & -\frac{1}{\sqrt{2}} \\
\frac{1}{\sqrt{2}} & \frac{1}{\sqrt{2}}
\end{array}\right),\left(\begin{array}{l}
0 \\
0
\end{array}\right), 0\right) .
$$

Actually, the $S$ also comes in handy for a single-channel case. In a situation when the distance between two systems, $G_{1}$ and $G_{2}$, is nonnegligible, the $(S, L, H)$ formalism can still be made to work if one inserts a phase shift $\phi$ between the two systems in the calculation. However, we must still assume that the time it takes to travel the distance in question is small compared to the timescale on which the systems evolve. The phase shift is inserted by placing the triplet $G_{\phi}=\left(e^{i \phi}, 0,0\right)$ between $G_{1}$ and $G_{2}$.

Another component one would like to incorporate in many setups is a coherent signal. Noting that this is just a displaced vacuum, one can show that a coherent signal, sending in $|\alpha|^{2}$ photons per second, is described by the triplet $G_{\alpha}=(1, \alpha, 0)$.

\section{$5.2(S, L, H)$ rules and examples}

The series product we derived above is the basis for the $(S, L, H)$ formalism. To allow for assembly and coupling of systems in many ways, we need to define two additional operations: concatenation ("stacking systems") and feedback (using an output from a system as an input for the same system). We also need to modify the series product to include $S$. In this section, we establish the full set of rules for all three $(S, L, H)$ operations and illustrate their use with examples from some of the appended papers.

\subsubsection{Series product, concatenation, and feedback}

The three operations of the $(S, L, H)$ formalism are illustrated in Fig. 5.2. The series product $\triangleleft$ from Eq. (5.2) generalizes, when including the scat- 

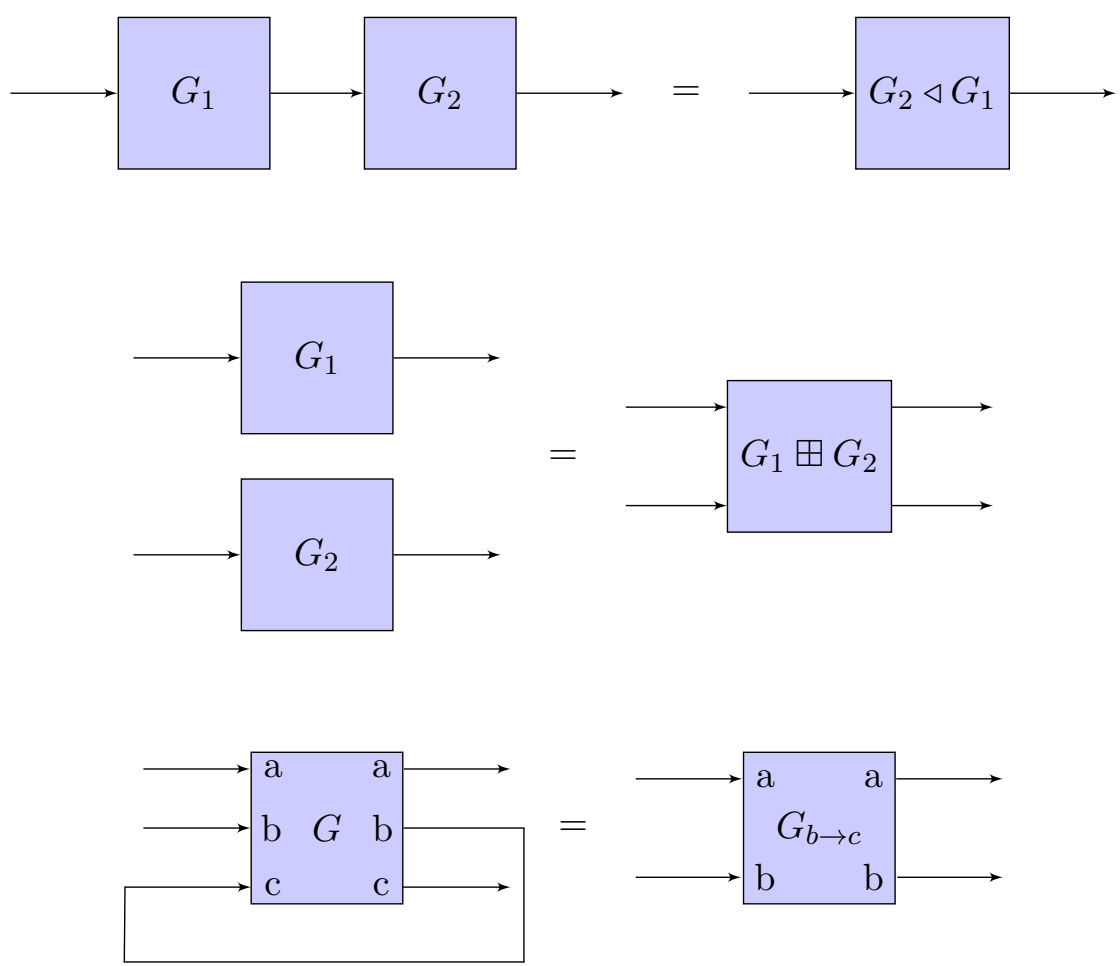

Figure 5.2: Illustrations of the three operations in the $(S, L, H)$ formalism. From top to bottom: series product $G_{2} \triangleleft G_{1}$, concatenation product $G_{1} \boxplus G_{2}$, and feedback.

tering matrix, to $[287,288]$

$$
G_{2} \triangleleft G_{1}=\left(S_{2} S_{1}, S_{2} L_{1}+L_{2}, H_{1}+H_{2}+\frac{1}{2 i}\left(L_{2}^{\dagger} S_{2} L_{1}-L_{1}^{\dagger} S_{2}^{\dagger} L_{2}\right)\right) .
$$

To assemble systems we also need the concatenation product $\boxplus$, which is given by

$$
G_{2} \boxplus G_{1}=\left(\left(\begin{array}{cc}
S_{2} & 0 \\
0 & S_{1}
\end{array}\right),\left(\begin{array}{l}
L_{2} \\
L_{1}
\end{array}\right), H_{2}+H_{1}\right) .
$$

Finally, there is also a rule for the feedback operation $[(S, L, H)]_{k \rightarrow l}=$ $(\tilde{S}, \tilde{L}, \tilde{H})$, which represents feeding the $k^{\text {th }}$ output of a system into the $l^{t h}$ 
input of the same system. The result is

$$
\begin{aligned}
\tilde{S} & =S_{[k, l]}+\left(\begin{array}{c}
S_{1, l} \\
\vdots \\
S_{k-1, l} \\
S_{k+1, l} \\
\vdots \\
S_{n, l}
\end{array}\right)\left(1-S_{k, l}\right)^{-1}\left(S_{k, l} \ldots S_{k, l-1} S_{k, l+1} \ldots S_{k, n}\right) \\
\tilde{L} & =L_{[k]}+\left(\begin{array}{c}
S_{1, l} \\
\vdots \\
S_{k-1, l} \\
S_{k+1, l} \\
\vdots \\
S_{n, l}
\end{array}\right)\left(1-S_{k, l}\right)^{-1} L_{k}, \\
\tilde{H} & =H+\frac{1}{2 i}\left(\left(\sum_{j=1}^{n} L_{j}^{\dagger} S_{j, l}\right)\left(1-S_{k, l}\right)^{-1} L_{k}-\text { H.c. }\right)
\end{aligned}
$$

where $S_{[k, l]}$ and $L_{[k]}$ are the original scattering matrix and coupling vector with row $k$ and column $l$ removed [289, 290].

Once we have the $(S, L, H)$ for our total system,

$$
G=\left(S,\left(\begin{array}{c}
L_{1} \\
\vdots \\
L_{n}
\end{array}\right), H\right)
$$

we can extract the master equation for the total system as

$$
\dot{\rho}=-i[H, \rho]+\sum_{i=1}^{n} \mathcal{D}\left[L_{i}\right] \rho .
$$

The average output from port $i$ of the system is simply given by $\left\langle L_{i}\right\rangle$.

\subsubsection{Example 1 - coupled cavities and a circulator}

For a first example of how to use the $(S, L, H)$ formalism, we will now look at a simplified version of a setup used in Paper I to model a bandpass 

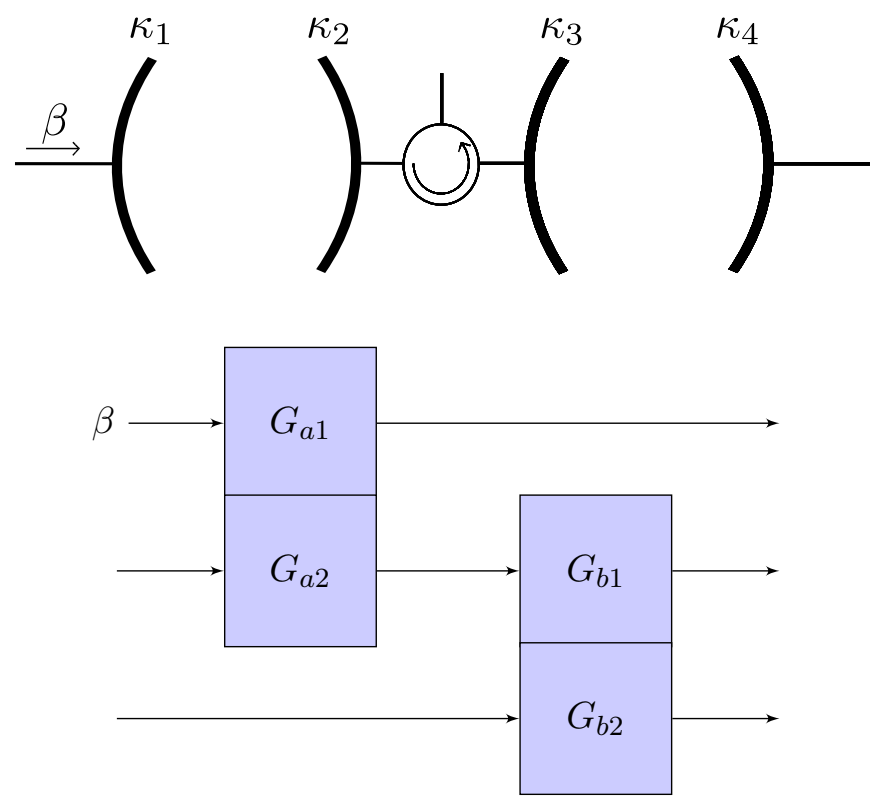

Figure 5.3: Top: Illustration of the setup for the two coupled cavities. The $\kappa_{i}$ denote photon loss rates through the cavity sides. Bottom: A schematic picture of the same setup, showing how to set up the $(S, L, H)$ triplet for the total system. It should be understood as follows: The uppermost input is that which is fed in through the left side of the first cavity, the middle input is that which enters the first cavity from the right, and the last input is that which enters the second cavity from the right. The first output is that which exits, or is reflected from, the left side of the first cavity. The second output is that which exits, or is reflected from, the left side of the second cavity. Finally, the last output exits, or is reflected from, the right side of the second cavity.

filter. In this version, depicted schematically in Fig. 5.3, a coherent signal impinges on a two-sided cavity from the left. The output from the right side of the cavity is then sent on, hitting a second two-sided cavity from the left. We imagine there being a circulator between the two cavities to prevent the signal reflected off the second cavity from returning and interacting with the first cavity. The circulator is assumed perfect and does not enter as a separate component in the $(S, L, H)$ calculations. In the case of an imperfect circulator, one can include it as a three-port device with a scattering matrix. This was done in Paper $\mathrm{V}$ to investigate the impact of circulator efficiency on the photon detection ability of cascaded transmons.

We begin by writing down the $(S, L, H)$ triplets of the system compo- 
nents shown in Fig. 5.3. The incoming coherent signal is simply represented by

$$
G_{\beta}=(1, \beta, 0),
$$

where $|\beta|^{2}$ is the photon flux, measured in units of photons per second. The first cavity has the triplet

$$
\begin{aligned}
G_{a} & =\left(\left(\begin{array}{ll}
1 & 0 \\
0 & 1
\end{array}\right),\left(\begin{array}{l}
\sqrt{\kappa_{1}} a \\
\sqrt{\kappa_{2}} a
\end{array}\right), H_{a}\right) \\
& =\left(1, \sqrt{\kappa_{1}} a, H_{a}\right) \boxplus\left(1, \sqrt{\kappa_{2}} a, 0\right) \equiv G_{a 1} \boxplus G_{a 2}
\end{aligned}
$$

where $a$ is the annihilation operator for the mode in the cavity, $\kappa_{1}$ and $\kappa_{2}$ are the photon loss rates through the left and the right side of the cavity, respectively, and

$$
H_{a}=\Delta_{a} a^{\dagger} a
$$

is the Hamiltonian of the cavity in a frame rotating with the frequency $\omega_{\beta}$ of the input signal; $\Delta_{a}=\omega_{a}-\omega_{\beta}$ is the detuning from the cavity frequency $\omega_{a}$. The decomposition of the cavity triplet using concatenation in Eq. (5.12) is useful since it lets us properly deal with using one of the cavity outputs as input for the second cavity, which has the triplet

$$
G_{b}=G_{b 1} \boxplus G_{b 2}=\left(1, \sqrt{\kappa_{3}} b, H_{b}\right) \boxplus\left(1, \sqrt{\kappa_{4}} b, 0\right),
$$

with everything defined in analogy with the case of the first cavity.

With the individual components in place, we now use the schematic in Fig. 5.3 together with the rules for concatenation and the series product to calculate triplet for the combined system,

$$
\begin{aligned}
G= & \left(I \boxplus G_{b 1} \boxplus G_{b 2}\right) \triangleleft\left(\left(G_{a 1} \triangleleft G_{\beta}\right) \boxplus G_{a 2} \boxplus I\right) \\
= & \left(\mathbf{1}_{3},\left(\begin{array}{c}
0 \\
\sqrt{\kappa_{3}} b \\
\sqrt{\kappa_{4}} b
\end{array}\right), H_{b}\right) \\
& \left.\triangleleft\left(\begin{array}{c}
\beta+\sqrt{\kappa_{1}} a \\
\sqrt{\kappa_{2}} a \\
0
\end{array}\right), H_{a}+\frac{1}{2 i} \sqrt{\kappa_{1}}\left(\beta a^{\dagger}-\beta^{*} a\right)\right) \\
= & \left(\mathbf{1}_{3},\left(\begin{array}{c}
\beta+\sqrt{\kappa_{1}} a \\
\sqrt{\kappa_{2}} a+\sqrt{\kappa_{3}} b \\
\sqrt{\kappa_{4}} b
\end{array}\right),\right. \\
& \left.H_{a}+H_{b}+\frac{1}{2 i} \sqrt{\kappa_{1}}\left[\beta a^{\dagger}-\beta^{*} a\right]+\frac{1}{2 i} \sqrt{\kappa_{2} \kappa_{3}}\left[a b^{\dagger}-a^{\dagger} b\right]\right) .
\end{aligned}
$$


Here, $\mathbf{1}_{n}$ denotes the $n \times n$ identity matrix and we inserted identity triplets $I=(1,0,0)$ in some places to make sure that the right channels are connected.

From Eq. (5.10) we now see that the master equation for the combined system is

$$
\begin{aligned}
\dot{\rho}= & -i\left[H_{a}+H_{b}+\frac{1}{2 i} \sqrt{\kappa_{1}}\left(\beta a^{\dagger}-\beta^{*} a\right)+\frac{1}{2 i} \sqrt{\kappa_{2} \kappa_{3}}\left(a b^{\dagger}-a^{\dagger} b\right), \rho\right] \\
& +\mathcal{D}\left[\beta+\sqrt{\kappa_{1}} a\right] \rho+\mathcal{D}\left[\sqrt{\kappa_{2}} a+\sqrt{\kappa_{3}} b\right] \rho+\mathcal{D}\left[\sqrt{\kappa_{4}} b\right] \rho .
\end{aligned}
$$

Noting that

$$
\begin{aligned}
\mathcal{D}[a+b] \rho= & \mathcal{D}[a] \rho+\mathcal{D}[b] \rho+a \rho b^{\dagger}+b \rho a^{\dagger} \\
& -\frac{1}{2}\left(\left(a^{\dagger} b+b^{\dagger} a\right) \rho+\rho\left(a^{\dagger} b+b^{\dagger} a\right)\right),
\end{aligned}
$$

after some algebra we are able to rewrite the master equation as

$$
\begin{aligned}
\dot{\rho}= & -i\left[H_{a}+H_{b}-i \sqrt{\kappa_{1}}\left(\beta a^{\dagger}-\beta^{*} a\right), \rho\right] \\
& +\left(\kappa_{1}+\kappa_{2}\right) \mathcal{D}[a] \rho+\left(\kappa_{3}+\kappa_{4}\right) \mathcal{D}[b] \rho \\
& +\sqrt{\kappa_{2} \kappa_{3}}\left(\left[b, \rho a^{\dagger}\right]+\left[a \rho, b^{\dagger}\right]\right) .
\end{aligned}
$$

The first two lines in this equation would be the full result if the two cavities were not connected; the third line contains the unidirectional coupling between the cavities. If we want to determine the signal that exits from the right side of the second cavity, we solve this master equation and use it to calculate $\sqrt{\kappa_{4}}\langle b\rangle$.

The motivation for including this type of setup in Paper I was to model a bandpass filter. To prove that a cavity acts as a filter for a signal, it is enough to study the steady state of the signal that is reflected from the first cavity, i.e., $\beta+\sqrt{\kappa_{1}}\langle a\rangle_{\mathrm{ss}}$ as given by the input-output relation Eq. (3.30). Using commutation relations for the $a$ 's and $b$ 's together with the cyclic property of the trace, we get that the steady state of $\langle a\rangle$ is given by

$$
0=\frac{\mathrm{d}}{\mathrm{d} t}\langle a\rangle=\operatorname{tr}(a \dot{\rho})=-\sqrt{\kappa_{1}} \beta-\left(i \Delta_{a}+\frac{1}{2} \kappa_{1}+\frac{1}{2} \kappa_{2}\right)\langle a\rangle,
$$

which leads us to

$$
\langle a\rangle_{\mathrm{ss}}=-\frac{\sqrt{\kappa_{1}} \beta}{i \Delta_{a}+\frac{1}{2} \kappa_{1}+\frac{1}{2} \kappa_{2}} .
$$


It is reassuring to note that the steady state of the first cavity does not depend in any way on the parameters of the second cavity, which is as it should be since the coupling is unidirectional. Assuming $\kappa_{1}=\kappa_{2} \equiv \kappa_{a}$, the steady-state reflected signal form the first cavity is thus

$$
\beta+\sqrt{\kappa_{a}}\langle a\rangle_{\mathrm{ss}}=\beta-\frac{\kappa_{a} \beta}{i \Delta_{a}+\kappa_{a}} .
$$

We see that the photon flux, the absolute value squared of the above quantity, is 0 when the input signal is on resonance with the cavity, i.e., when $\Delta_{a}=0$. Furthermore, the photon flux is exactly half of the input when $\Delta_{a}= \pm \kappa_{a}$. This shows that the cavity acts as a filter with bandwidth $2 \kappa_{a}$.

\subsubsection{Example 2 - a giant artificial atom}

For a second $(S, L, H)$ example, we turn to the analysis of the giant artificial atom in Paper VII. There, we consider a multi-level atom coupled to an open transmission line at multiple points. The most basic version of this setup is a two-level atom coupled at two points to the transmission line. In the $(S, L, H)$ formalism, we can handle this system by letting the output from the first coupling point pass through a phase shift (accounting for the distance between coupling points, assuming that the travel time is negligible) and then be fed back to the atom at the second coupling point.

The setup we consider, together with its $(S, L, H)$ model, is depicted in Fig. 5.4. We first look at the part of the atom interacting with the right-travelling modes. This part has an $(S, L, H)$ triplet $G_{\mathrm{R}}$, which can be divided into two, $G_{\mathrm{R}, 1}$ and $G_{\mathrm{R}, 2}$, one for each connection point. Including the Hamiltonian $\frac{\Delta_{\mathrm{a}}}{2} \sigma_{z}$, where $\Delta_{\mathrm{a}}=\omega_{\mathrm{a}}-\omega_{\mathrm{p}}$ is the detuning of the atom transition frequency from some probe frequency $\omega_{\mathrm{p}}$, in $G_{\mathrm{R}, 1}$, we have

$$
\begin{aligned}
G_{\mathrm{R}, 1} & =\left(1, \sqrt{\frac{\Gamma_{1}}{2}} \sigma_{-}, \frac{\Delta_{\mathrm{a}}}{2} \sigma_{z}\right), \\
G_{\mathrm{R}, 2} & =\left(1, \sqrt{\frac{\Gamma_{2}}{2}} \sigma_{-}, 0\right),
\end{aligned}
$$

where $\Gamma_{k}$ is the relaxation rate of the atom via connection point $k$. The $\Gamma_{k}$ are divided by 2 here since only half of the relaxation goes to the righttravelling modes. 

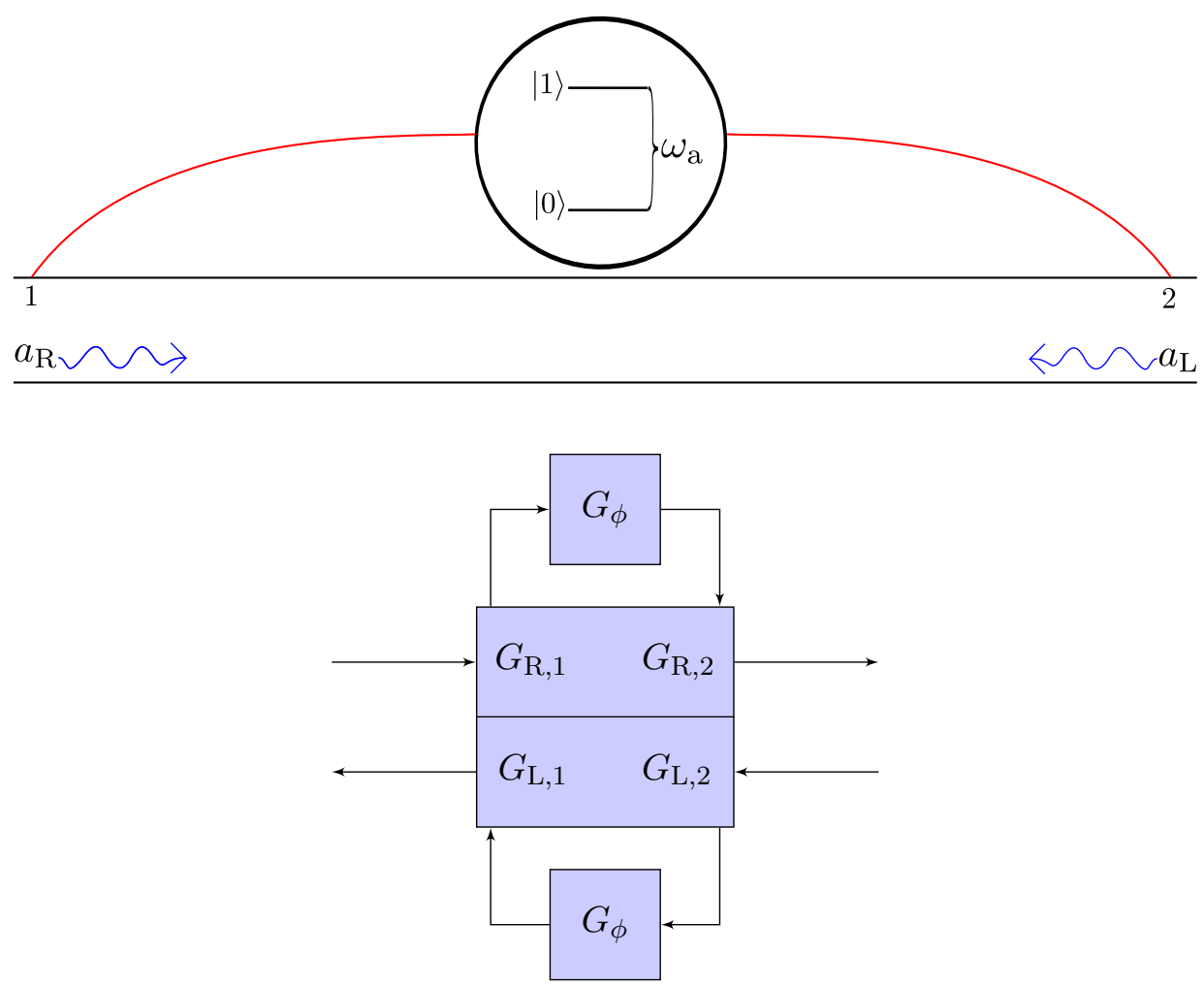

Figure 5.4: Top: Illustration of the setup for the giant artificial atom. The atom is coupled at two points to the transmission line, which supports right- and lefttravelling modes. Bottom: A schematic picture of the same setup, showing how to set up the $(S, L, H)$ triplet for the total system. The atom is conceptually divided into two parts, one interacting with each direction of the travelling modes. The output of each such mode from its first interaction point is fed through a phase shift and the returns to the atom at the second interaction point.

We now find $G_{\mathrm{R}}$ from the calculation

$$
\begin{aligned}
G_{\mathrm{R}} & =\left[\left(G_{\phi} \triangleleft G_{\mathrm{R}, 1}\right) \boxplus G_{\mathrm{R}, 2}\right]_{1 \rightarrow 2} \\
& =\left(\left(\begin{array}{cc}
e^{i \phi} & 0 \\
0 & 1
\end{array}\right),\left(\begin{array}{c}
e^{i \phi} \sqrt{\frac{\Gamma_{1}}{2}} \sigma_{-} \\
\sqrt{\frac{\Gamma_{2}}{2}} \sigma_{-}
\end{array}\right), \frac{\Delta_{\mathrm{a}}}{2} \sigma_{z}\right)_{1 \rightarrow 2} \\
& =\left(e^{i \phi},\left(e^{i \phi} \sqrt{\frac{\Gamma_{1}}{2}}+\sqrt{\frac{\Gamma_{2}}{2}}\right) \sigma_{-}, \frac{1}{2} \sigma_{z}\left(\Delta_{\mathrm{a}}+\frac{1}{2} \sqrt{\Gamma_{1} \Gamma_{2}} \sin (\phi)\right)\right),
\end{aligned}
$$


where we used all the rules from Eqs. (5.4)-(5.8) and $e^{i \phi}-e^{-i \phi}=2 i \sin (\phi)$. In the same way, we get

$$
\begin{aligned}
G_{\mathrm{L}} & =\left[\left(G_{\phi} \triangleleft G_{\mathrm{L}, 2}\right) \boxplus G_{\mathrm{L}, 1}\right]_{1 \rightarrow 2} \\
& =\left(e^{i \phi},\left(e^{i \phi} \sqrt{\frac{\Gamma_{2}}{2}}+\sqrt{\frac{\Gamma_{1}}{2}}\right) \sigma_{-}, \frac{1}{4} \sigma_{z} \sqrt{\Gamma_{1} \Gamma_{2}} \sin (\phi)\right),
\end{aligned}
$$

and thus the total triplet for our system is

$$
\begin{aligned}
G_{\mathrm{tot}} & =G_{\mathrm{R}} \boxplus G_{\mathrm{L}} \\
& =\left(\left(\begin{array}{cc}
e^{i \phi} & 0 \\
0 & 1
\end{array}\right),\left(\left(\begin{array}{c}
\left.e^{i \phi} \sqrt{\frac{\Gamma_{1}}{2}}+\sqrt{\frac{\Gamma_{2}}{2}}\right) \sigma_{-} \\
\left.e^{i \phi} \sqrt{\frac{\Gamma_{2}}{2}}+\sqrt{\frac{\Gamma_{1}}{2}}\right) \sigma_{-}
\end{array}\right), \frac{\Delta_{\mathrm{a}}+\sqrt{\Gamma_{1} \Gamma_{2}} \sin (\phi)}{2} \sigma_{z}\right) .\right.
\end{aligned}
$$

With the total triplet in hand, Eq. (5.10) gives us the master equation. With the simplifying assumption $\Gamma_{1}=\Gamma_{2} \equiv \Gamma$, the result is

$$
\dot{\rho}=-i\left[\frac{\Delta_{\mathrm{a}}+\Gamma \sin (\phi)}{2} \sigma_{z}, \rho\right]+\left|1+e^{i \phi}\right|^{2} \Gamma \mathcal{D}\left[\sigma_{-}\right] .
$$

Thus, the effect of the feedback in the giant artificial atom is to introduce a shift of the resonance frequency by $\Gamma \sin (\phi)$ (a Lamb shift) and to give an effective relaxation rate $\left|1+e^{i \phi}\right|^{2} \Gamma$. Both these modifications depend on the phase shift $\phi$, which is determined by the distance between the coupling points and the resonance frequency of the atom. This means that we have a frequency-dependent coupling, which is discussed further in Paper VII. There, we also do the derivation in the traditional master equation way (see Sec. 3.1), which gives a more precise account for the Lamb shift.

The method used here for the giant atom is also applicable to the case of an atom placed in front of a mirror. In that case, the right-travelling output from the atom gets a phase shift as it travels to the mirror and back, and is then fed back to the atom as the left-travelling input. In this case one also gets a frequency and distance dependence of the relaxation rate and the atom frequency. The varying relaxation rate is investigated experimentally in Paper VIII; it can be interpreted as a measure of the vacuum fluctuation strength in the transmission line, and this strength varies since the mirror imposes a boundary condition giving nodes and anti-nodes for the vacuum modes. 
The $(S, L, H)$ formalism concludes our overview of the theoretical methods used in the appended papers. With the full theoretical toolbox from Chapters $2-5$ in hand, we are now ready to take a closer look at the appended papers in the following chapter. 


\section{Chapter 6}

\section{Paper overview}

In this penultimate chapter, we give an overview of the eight appended papers upon which this thesis is based. The focus of the overview is on explaining the main ideas of the papers and showing how the theoretical methods of the previous chapters are applied in practice. Broadly put, all the appended papers are theoretical or experimental studies of systems where artificial atoms enable us to either improve on known concepts or experiments from quantum optics with natural atoms, or to explore new regimes which have not been possible to reach with natural atoms.

Little research is done in isolation these days; all the appended papers are collaborations between several researchers. As a part of the paper overview, I will briefly clarify my own contribution to each of the appended papers.

\subsection{Paper I - Undoing measurement-induced de- phasing in circuit QED}

In Sec. 1.4, we discussed how parity measurements on qubits are an integral part of many error correction schemes for quantum computing. A proposal for how to carry out a 2-qubit parity measurement in circuit QED was presented in 2010 [165] (there are now also similar schemes for measuring 3 - and 4-qubit parity [291, 292], and even to measure $N$-qubit parity using an ancilla qubit [293]). The idea is to place two qubits in a cavity, tune the system into the dispersive regime (see Sec. 2.5), and send in a coherent microwave signal at the resonance frequency of the cavity. The dispersive shifts of the qubits can then be arranged such that if the qubits 
are in opposite states their effects on the cavity resonance frequency cancel, while if they are in identical states they will cooperate to shift the cavity frequency up or down. Performing homodyne detection on one quadrature of the outgoing signal will only reveal whether it is on resonance with the cavity or not, thus only giving information about the parity of the two qubits (and no information about their individual states). However, akin to the example in Sec. 4.1.1, the parity measurement comes with additional back-action in the form of phase kicks to the states in one of the parity subspaces. Averaging over many measurements, these phase kicks look like measurement-induced dephasing.

In Ref. [216], Tornberg and Johansson showed that part of this dephasing can be undone. It turns out that monitoring the homodyne current (the measurement signal, see Sec. 4.3) gives information about the phase kicks, similar to the example in Sec. 4.1.1. However, it appeared that only part of the information about the phase kicks could be extracted this way, and thus the problem of measurement-induced dephasing remained.

In Paper I, we look at measurements on both one and two qubits in a cavity, using both homodyne detection and photon detection in the SME formalism of Secs. 4.2 and 4.3. We show that all the information about phase kicks can be extracted from the measurement signal in all these cases, and thus that the measurement-induced dephasing in principle can be completely undone.

The key insight for the positive result of Paper I was that Ref. [216] considered the steady-state case, i.e., the coherent probe is turned on at time $t=0$ and is never turned off. We try to undo the phase kicks we have information about, once a long time has passed. The problem with this approach is that there will always be probe photons left in the cavity which have yet to leak out and reach the detector. Each such remaining probe photon is entangled with the qubits and carries information about their phase, which we need to completely undo the dephasing. The solution is simple: we analyze the situation where the probe signal has been turned off at a time $t=t_{\text {off }}>0$, and we have waited some time after that to let the remaining photons leak out of the cavity and reach the detector.

In this way, we acquire all information about the phase kicks. For the case of homodyne detection, the information consists of the homodyne current from time $t=0$ and onwards, while for the case of photon detection it is all the times when a photon was detected. The calculation for the case of photon detection, including tracing out the cavity degrees of freedom 
to derive an effective SME for one qubit, has not been done previously to our knowledge. The effective SME for homodyne detection has been derived before [165, 215], but the result that all the measurement-induced dephasing can be undone if the probe is turned off, is new.

The positive result on undoing the phase kicks completely is, of course, only achieved in the limit of perfect detectors. Presently, there are no efficient photon detectors for propagating microwave photons and the homodyne detection schemes struggle with amplifier noise, as discussed in Sec. 4.3. To see if our results could be tested with currently available experimental equipment, we model the case of homodyne detection with imperfect detectors and limited bandwidth. We use the $(S, L, H)$ formalism of Chapter 5 to insert a second cavity, acting as a bandpass filter (see Sec. 5.2.2) through which the output from the cavity with the qubits was passed. According to the results from this model, it should be feasible to demonstrate some undoing of measurement-induced dephasing already with existing technology. This year, such an experimental confirmation of our results was achieved in Delft [294].

For Paper I, my contribution consisted of deriving the equations used, performing the numerical simulations, interpreting results, and writing most of the manuscript.

\subsection{Paper II - Breakdown of the cross-Kerr scheme for photon counting}

In Sec. 1.1, we saw that one of the missing experimental tools for quantum optics with superconducting circuits is a good detector of propagating photons at microwave frequencies, although there are proposals to remedy this [51-55]. Paper II is an investigation of the possiblity to use a threelevel transmon to mediate a cross-Kerr type interaction between photons to construct a photon detector. Generally, a Kerr interaction between two modes with annihilation operators $a$ and $b$, respectively, is of the form $\chi_{K} a^{\dagger} a b^{\dagger} b$. If the interaction strength $\chi_{\mathrm{K}}$ is large enough, the presence of photons in one mode will change the frequency of the photons in the other mode, giving them a phase shift.

In our approach, a single photon, which we wish to detect, is close to resonance with the first transition in the transmon. The idea is that the arrival of this photon at the transmon will induce a phase shift in a coherent probe, which is close to resonance with the second transition of 
the transmon. This phase shift can then be read out from a homodyne detection on the probe. The question is whether the shift is large enough to be discernible over the unavoidable vacuum noise.

We model the system at hand in two different ways. In the first approach, we place a cavity, which will be our photon source, to the left of the transmon. Using the $(S, L, H)$ formalism of Chapter 5 , we derive the master equation for the total system. Then, adding a homodyne measurement on the coherent probe after it has interacted with the transmon gives us a stochastic master equation (see Sec. 4.3). By numerically simulating many quantum trajectories, some starting with one photon in the cavity and some with zero photons in the cavity, we get a distribution of measurement results (integrated homodyne currents) for each case. From the separation and widths of the two distributions we extract the signal-tonoise ratio (SNR).

The second approach is to use the Fock-state input-output formalism of Sec. 3.4. Here, it is more straightforward to consider different shapes of the photon wavepacket. Also, it turns out that the system of equations for the density matrix is analytically solvable. We are thus able to extract the SNR in a different way than before; the result is the same as in the first approach.

The main result of the paper is that a single transmon can not give an SNR above 1, which means that it will not function as a good photon detector. Even putting several transmons one after another in the transmission line does not help. If they are too close together, they will act as a single transmon; if they are far apart, the SNR is still below 1 since a large part of the probe and the signal are reflected off the first transmons and does not propagate onwards. Other ideas, such as squeezing the probe field and varying the ratio of the two transmon relaxation rates, also fail to produce a good SNR. The basic problem is that the transmon can only handle one photon at a time, which means that the cross-Kerr interaction only shifts the probe signal by an amplitude of "less than one photon", no matter how strong the probe is. This is not enough to clearly distinguish a signal above the vacuum noise, which can be said to have the amplitude of half a photon.

For Paper II, I contributed by collaborating on deriving equations and performing numerical simulations, and by discussing ideas and results. I also assisted in the writing of the manuscript. 


\subsection{Paper III - Giant cross-Kerr effect for prop- agating microwaves induced by an artificial atom}

Paper III is an experimental investigation of the cross-Kerr effect we tried to use for photon detection in Paper II. Two setups are tested: a single three-level transmon in an open transmission line and a transmon at the end of a transmission line. In both cases two coherent signals are sent in: one, the "control", close to resonance with the first transmon transition, and another, the "probe", close to resonance with the second transition. See Fig. 1.1 for an illustration of the open transmission line case.

As was mentioned in Sec. 1.1, similar setups using natural atoms have only achieved a weak photon-photon interaction, where a single control photon only imparts a phase shift of less than a milliradian on the probe [31-33]. In Paper III, we report as our main result phase shifts of tens of degrees when both the probe and the control are on the single-photon level. By single-photon level for the coherent signals, we mean a signal power such that on average one photon arrives at the transmon per relaxation time. An important thing to note is that the phase shift is larger when the transmon sits at the end of a transmission line. This is due to the fact that all signals only travel one way in this setup; in an open transmission line, the transmon output splits up with half of it leaving in each of the two possible propagation directions.

The behaviour of the transmon in the experiment is well explained by a model where the decoherence is mainly due to relaxation to the transmission line. The model uses a master equation and input-output theory as given in Chapter 3. In Paper II we assume the idealized case where relaxation to the transmission line is the only decoherence mechanism. While the setup in Paper II will not work as a photon detector, an improved version of it considered in Paper V will. The results of Paper III give us good reason to believe that the setup of Paper $\mathrm{V}$ can be made to work in practice.

In the work on Paper III, I had the main responsibility for the theoretical part. I also assisted in fitting the data and gave input on the writing of the manuscript. 


\subsection{Paper IV - Detailed modelling of the sus- ceptibility of a thermally populated, strongly driven circuit-QED system}

In Paper IV, we look at results from an experiment where a transmon was placed in a resonator. This setup realizes the Jaynes-Cummings model discussed in Sec. 2.5, but with a multi-level atom rather than a two-level one.

To probe the system, a weak coherent signal is sent into the resonator and its transmission coefficient is measured. With the first transmon transition frequency relatively close to the resonance frequency of the cavity, transmission peaks are observed at frequencies corresponding to transitions between dressed states (see Sec. 2.5).

A strong coherent drive is then applied close to the first transmon transition. The result is that we get doubly dressed states, where both the resonator and the drive interact with the transmon. As the frequencies of both probe and drive are varied, a rich pattern of resonances appear. We are able to model the experimental results with good precision for a range of drive strengths using a master equation (including a thermal population of the bath, see Sec. 3.1) and calculating the susceptibility for the weak probe (see Sec. 4.4). The features in the data can all be qualitatively explained in terms of the doubly dressed states and multi-photon transitions between them.

For Paper IV, I did most of the theoretical work. I set up the model, wrote code for numerical simulations, and explained qualitatively the features in the data. I also assisted in writing the manuscript.

\subsection{Paper V-Quantum nondemolition detection of a propagating microwave photon}

Paper V is a theoretical study building on Paper II in the search for a detector of propagating microwave photons. While we found in Paper II that the effective photon-photon interaction mediated by a single threelevel transmon was too weak to allow for photon detection, we did not give up on the idea. Paper II also showed that cascading several transmons in an open transmission line did not help, due to problems with reflections, but in Paper V we hit upon a setup which avoids these problems. 


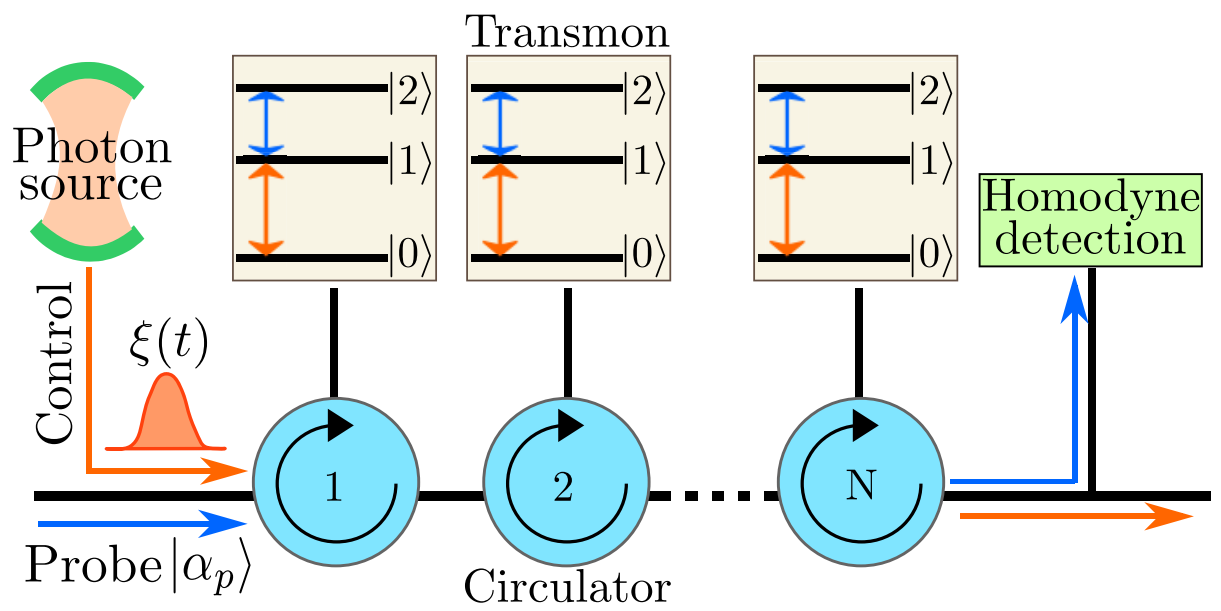

Figure 6.1: A schematic of the photon-detection setup considered in Paper V. The photon wavepacket $\xi(t)$ to be detected (close to resonance with the first transmon transition) and the coherent probe of amplitude $\alpha_{\mathrm{p}}$ (close to resonance with the second transmon transition) are guided by circulators to each transmon in turn. This adds up the effective photon-photon interactions, giving a clear difference in the probe depending on whether there was a photon present or not. Illustration by Sankar R. Sathyamoorthy.

The setup studied in Paper V is shown in Fig. 6.1. By placing each transmon at the end of a transmission line, and connecting the lines with circulators, we force both the photon wavepacket and the coherent probe to travel unidirectionally. The main result of the paper is that this makes the effective photon-photon interactions add up to give a $\sqrt{N}$ increase in SNR for $N$ transmons in the chain. Just like in Paper II, the analysis is based on an SME for homodyne detection, the $(S, L, H)$ formalism to handle cascaded quantum systems, and the formalism for Fock-state input. An important point compared to other photon-detector proposals is that the photon we detect is not absorbed, but travels on, making the detection "nondemolition".

We analyze a number of possible imperfections in the setup that could affect an experimental implementation of the proposal. The effects of measurement efficiency for the homodyne detection of the probe, the shape of the photon wavepacket, dephasing, varying coupling strengths, and losses in the circulators are all considered. With the promising developments regarding measurement efficiency referred to in Sec. 4.3, the most critical 
issue is probably the circulators. We find that a $5 \%$ power loss in each circulator can be tolerated in the setup, but with power loss approaching $10 \%$ the SNR will not increase no matter how many transmons are added to the chain.

While we have yet to see an experimental implementation of the proposal in Paper $\mathrm{V}$, there is a recent paper which seeks to improve further on the setup [295]. There, each transmon is placed in a cavity. The photon to be detected travels the same path as before, but each cavity is probed by a separate coherent signal. This appears to reduce the number of transmons needed to achieve good SNR.

For Paper V, my main contribution was to derive most of the equations in the theoretical models. I also assisted in some numerical calculations, discussed the results, and gave input on the manuscript.

\subsection{Paper VI - Propagating phonons coupled to an artificial atom}

Paper VI is an experiment which opens up new realms in quantum optics. Here, we make phonons in the form of surface acoustic waves (see Sec. 2.4) interact with an artificial atom, a transmon. The idea for this setup was first given in Ref. [204]. With the SAWs propagating on a piezoelectric substrate, the interdigitated shunt capacitance of the transmon (see Sec. 2.3) can be designed to act as an IDT, converting between electrical excitations of the atom and propagating phonons.

We perform several tests with the experimental setup in Fig. 1.2 to confirm that the artificial atom couples to the SAWs. The tests are all inspired by the similarity of the setup with a transmon in an open transmission line. Firstly, the reflection coefficient for a SAW probe sent towards the transmon is shown to be power-dependent, consistent with the fact that the anharmonic artificial atom can only handle one phonon at a time. Secondly, driving the transmon electrically while listening for its emission of SAWs gives results consistent with multi-phonon emission at the transmon transition frequencies. Furthermore, the slow propagation velocity of the SAWs, about $2900 \mathrm{~m} / \mathrm{s}$, lets us see that the SAW emission from the transmon arrives at our detector roughly $40 \mathrm{~ns}$ after the electrical drive is turned on. This is consistent with the distance between the transmon and the detector. Thirdly, we perform a hybrid two-tone spectroscopy on the transmon where we drive it electrically and probe it via SAWs. This 
is similar to the experiment in Paper IV; we observe resonances that we can identify with the multi-level structure of the transmon dressed by the electrical drive.

The experiments are modelled with a master equation and input-output theory from Chapter 3. For the two-tone spectroscopy, we also use the susceptibility of Sec. 4.4. In the experiment, the transmon can be seen to couple to the phonons at several points (each finger of the IDT structure) that are on the order of wavelengths apart. In Paper VII, we show that this leads to a frequency-dependent relaxation rate, but since we only ever listen to the SAWs at a single frequency, it is not necessary to include the frequency-dependence in the model to explain the experimental data.

For Paper VI, I contributed by setting up the quantum model, writing code for numerical simulations, and assisting in the data fitting. I also assisted in the interpretation of results and gave input on the manuscript.

\subsection{Paper VII - Designing frequency-dependent relaxation rates and Lamb shifts for a giant artificial atom}

Inspired by the breakthrough in Paper VI, Paper VII is a theoretical study of a giant artificial atom coupled to an open 1D environment. As we explained in Sec. 2.4.2, both natural and artificial atoms are usually very small compared to the wavelength of the photons (or phonons) they interact with. Therefore, there has until the arrival of paper VI not been much reason to study "giant" atoms.

The theoretical model we employ for the giant artificial atom is shown in Fig. 2.9 and discussed in Sec. 2.4.2. We consider an atom which couples to left- and right-moving $1 \mathrm{D}$ fields at a number of points that can be far enough apart for the field to get a sizable phase shift travelling from one point to the next. However, we assume the travel time itself to be negligible compared to the timescale set by the atom relaxation time. Relaxing this assumption is an interesting direction for future work.

We set up a Hamiltonian for the atom plus the 1D fields (the environment), including the aforementioned phase shifts, and then proceed in the fashion of Sec. 3.1 and Appendix B to derive a master equation for the multi-level atom (we also do an $(S, L, H)$ calculation similar to that of Sec. 5.2.3). This leads to the two main results of the paper. Firstly, 
the atom relaxation rate becomes frequency-dependent. The distance between coupling points leads to different phase shifts, and thus either constructive or destructive interference, for different frequencies. This means that by choosing the distance between coupling points, one can design the frequency-dependence of the coupling to suit whatever needs one might have. For example, it is possible to arrange things such that the first transition of the transmon relaxes slowly, while the second transition relaxes fast. By driving the transmon to the second excited state, it should then be possible to create population inversion.

Secondly, the interference between coupling points also leads to a more complicated expression for the Lamb shift than for a "small" atom, since the virtual photons (vacuum fluctuations) that create this frequency renormalization can now interact with the atom in several places. Of course, the Lamb shift can now also be designed by choosing the coupling points coordinates appropriately.

Beside the theoretical analysis, Paper VII also proposes a second possible experimental implementation of a giant artificial atom besides that seen in Paper VI. We believe one could use a variation of the transmon design, called the "xmon" [200] to couple to an ordinary superconducting transmission line at several points. By meandering the transmission line in a suitable way, the distance between coupling points could be on the order of wavelengths.

For Paper VII, my contribution consisted of setting up the theoretical model, deriving the equations, performing numerical simulations, discussing the results, and writing the manuscript.

\subsection{Paper VIII - Probing the quantum vacuum with an atom in front of a mirror}

Paper VIII can be considered an experimental demonstration of a precursor to the case of multiple coupling points discussed in Paper VII. In Paper VIII, a transmon is placed at a distance from the end of a transmission line as sketched in Fig. 1.3. The end of the transmission line acts as a mirror, and thus the transmon interacts twice with any incoming signal; once when it first hits the transmon and then again when it returns to the transmon after having reflected off the mirror. This leads to interference effects just like in Paper VII. The transition frequency of the artificial atom sets the distance to the mirror in units of wavelength and determines 
whether there will be constructive or destructive interference, giving strong or weak coupling between the atom and the transmission line.

Another view of the situation is that the mirror is a boundary condition which imposes a mode structure on the vacuum in the transmission line. If the atom transition frequency corresponds to an antinode of this structure, the atom will couple strongly to the line and relax quickly. If instead the atom sits at a node, it will relax slowly. By sending in a coherent probe at different frequencies, we can measure the atom relaxation rate. The relaxation rate is proportional to the spectral density of the vacuum fluctuations in the transmission line (see Sec. 2.2). In the experiment, we can vary the transmon transition frequency and thus map out the strength of the vacuum fluctuations in the vicinity of the mirror.

The structure of the vacuum in cavities have been measured before in various systems [296, 297], but here we have a semi-infinite transmission line instead. The results we get are also much cleaner than previous efforts with natural atoms in front of a mirror [34], as discussed in Sec. 1.1.

We measure a change in relaxation rate of the atom of about an order of magnitude as we tune its transition frequency close to a node. The lowest value of the spectral density of the vacuum fluctuations that we observe is $0.02 \hbar \omega$, which should be compared with the expected value of $\hbar \omega$ in an open transmission line or $2 \hbar \omega$ at an antinode in front of a mirror.

The experimental results are modelled with a master equation and the input-output theory of Chapter 3. To arrive at the frequency-dependence of the coupling, using an $(S, L, H)$ derivation similar to that of Sec. 5.2.3 is an easy route.

For Paper VIII, I contributed by collaborating on setting up the theoretical model and discussing the results. I also assisted in the writing of the manuscript. 



\section{Chapter 7}

\section{Summary and outlook}

In this thesis, we have studied a number of systems where artificial atoms enable us to either improve on known concepts or experiments from quantum optics with natural atoms, or explore new regimes which have not been possible to reach with natural atoms. Chapter 2 covered the systems considered: artificial atoms and transmission lines, made from superconducting circuits, and surface acoustic waves. The theoretical tools of circuit quantization, master equations, input-output theory, stochastic master equations for quantum measurements, and the $(S, L, H)$ formalism for cascaded quantum systems were reviewed in Chapters $2-5$.

The focus of the first appended papers is quantum measurements. In Paper I, we show how unwanted measurement back-action (in the form of measurement-induced dephasing) can be undone when probing one or two qubits dispersively coupled to a resonator. The results apply to both homodyne detection and photon detection, and are relevant for parity measurements, which are needed to implement error-correcting surface codes for quantum computing. Recently, the effect was confirmed in an experiment [294].

Papers II, III, and V treat the problem of detecting propagating photons at microwave frequencies. We consider a setup where artificial threelevel atoms mediate an effective photon-photon interaction between the propagating signal we want to detect and a coherent probe. In Paper II, we show that although the artificial atom can mediate a quite strong interaction, it is not enough to overcome the fundamental quantum noise limitations and make the setup work as a photon detector. However, in Paper $\mathrm{V}$ we then show that by cascading a number of three-level atoms 
in the right way, we can overcome the vacuum noise and achieve a good signal-to-noise ratio. An important advantage of our system compared to the photomultipliers for optical photons is that the setup with three-level atoms is nondestructive, i.e., the photon can propagate onward after being detected. Paper III is an experimental study of the photon-photon interaction we can mediate with a three-level atom. We show that we can impart a phase shift of tens of degrees per photon when both the signal and the probe are at the single-photon level. This is several orders of magnitude larger than what has been achieved for propagating photons in other systems with natural atoms.

Looking to the future, it will be interesting to see whether the parity measurement and photon detection schemes discussed here will find practical applications. For the photon detection, one could investigate whether the setup can be made to resolve photon numbers as well, not only the absence or presence of photons. As shown in Ref. [295], cavities could be used to further improve the detection efficiency.

In Paper IV, we study experimental results for an artificial atom coupled to photons in a cavity. The atom is subject to a strong drive signal and the cavity is probed with a weak signal. We are able to explain the rich dynamics exhibited by the system, involving multi-photon transitions, in terms of dressed states formed by the atom and the cavity photons, and in terms of doubly dressed states which occur when the aforementioned dressed states interact with the strong drive on the atom.

Papers VI, VII, and VIII investigate an artificial atom coupled to a bosonic field at several points, spaced wavelengths apart. This is a new regime which has not been reachable in quantum optics with natural atoms. Paper VI is an experimental demonstration of coupling between an artificial atom (a transmon) and phonons in the form of surface acoustic waves. The low phonon propagation velocity makes the phonon wavelength much shorter than that of microwave photons, allowing us to leave the regime where the atom can be considered point-like compared to the field it interacts with. Paper VII is a more in-depth analysis of the theory for this new type of system. There, we find that the multiple coupling points between atom and field allow us to design frequency-dependent coupling strength and energy shifts for the "giant artificial atom". Finally, in Paper VIII, an artificial atom placed in front of a mirror is used as a probe of the interference pattern that arises in the mode structure of the quantum vacuum fluctuations due to the presence of the mirror. 
The coupling between artificial atoms and surface acoustic waves demonstrated in Paper VI offer many possibilities for further work [298]. For example, a slow-moving phonon could be caught between two artificial atoms. The many coupling points in the SAW-transmon setup should not only allow us to test predictions from Paper VII, they could also be a route to the regime of ultrastrong coupling (see Sec. 2.5). Noting that the phonon wavelength at microwave frequencies matches that of optical photons, it is also worthwhile to consider whether this could be used for conversion between optical photons and microwave excitations; this is an active area of investigation in some other setups [299, 300]. Finally, there has also been some work on building networks for quantum information using phonons rather than photons [301].

The theoretical work on the giant artificial atom in Paper VII introduces a new timescale compared to the situation with a small atom. This timescale, the time it takes to travel between coupling points, was assumed to be small compared to the atom relaxation time in Paper VII, but this assumption could be done away with. There is some previous work on an atom placed far from a mirror which could guide such an effort [302]. It would also be interesting to explore the regime where the coupling strength at a single finger, or the total coupling strength, is ultrastrong.

The internal structure of a giant artificial atom also opens up new possibilities for combining quantum systems. For example, the classic setup from Sec. 2.5 with an atom in a cavity could be turned on its head; we can now place a cavity in an atom. Whether this or other setups, e.g., an atom inside an atom, give rise to new and interesting physics remains to be seen.

Taking a broader look at quantum optics with artificial atoms, hybrid systems seem to be on the rise, as discussed in Sec. 1.2. Combining SAWs and superconducting circuits as in Paper VII is just one example. In the same vein, it could be possible to couple a transmon to graphene plasmons.

Finally, input-output theory and the $(S, L, H)$ formalism of Chapter 5 are still being developed further, e.g., to include non-Markovian networks [303]. Combining these theoretical tools with experimental advances for superconducting circuits and well-developed software for numerical calculations $[289,304,305]$ makes it feasible to consider larger setups with many artificial atoms and resonators [306-309].

All in all, we have used artificial atoms to break new ground in quantum optics, but we are only starting to tap the vast potential of this research field. 



\section{Appendix A}

\section{Transformations}

In this appendix, we give the details of two important unitary transformations: the dispersive transformation and the transformation to a rotating frame. The first is given in Eq. (2.77), and lets us simplify the JaynesCummings Hamiltonian in Eq. (2.71) to Eq. (2.78). The second is useful to eliminate time dependence and/or clarify which terms can be thrown away when applying the RWA.

\section{A.1 Properties and identities for unitary trans- formations}

We consider a unitary transformation $U$. If this transformation changes a system state $|\psi\rangle$ into $|\tilde{\psi}\rangle$ according to

$$
|\tilde{\psi}\rangle=U|\psi\rangle,
$$

we can use that the Schrödinger equation should remain in the same form after the transformation, i.e.,

$$
\begin{aligned}
i \frac{\mathrm{d}}{\mathrm{d} t}|\psi\rangle & =H|\psi\rangle, \\
i \frac{\mathrm{d}}{\mathrm{d} t}|\tilde{\psi}\rangle & =\tilde{H}|\tilde{\psi}\rangle,
\end{aligned}
$$

to infer that the transformed Hamiltonian $\tilde{H}$ should be given by

$$
\tilde{H}=U H U^{\dagger}+i \dot{U} U^{\dagger} .
$$


Many unitary transformations are written as exponentials of operators. Therefore, in calculations it is useful to know the Baker-Hausdorff lemma [104]

$$
\exp (\lambda G) A \exp (-\lambda G)=A+\lambda[G, A]+\frac{\lambda^{2}}{2 !}[G,[G, A]]+\ldots,
$$

where $G$ and $A$ are operators and $\lambda$ is a scalar. Applying this to our system of photons and an atom, it also good to know the commutation relations

$$
\begin{aligned}
{\left[a, a^{\dagger}\right] } & =1, \\
{\left[a, a^{\dagger} a\right] } & =a, \\
{\left[a^{\dagger}, a^{\dagger} a\right] } & =-a^{\dagger}, \\
{\left[\sigma_{-}, \sigma_{+}\right] } & =-\sigma_{z}, \\
{\left[\sigma_{-}, \sigma_{z}\right] } & =2 \sigma_{-}, \\
{\left[\sigma_{+}, \sigma_{z}\right] } & =-2 \sigma_{+} .
\end{aligned}
$$

The last three are easily derived using $\sigma_{-}=|g\rangle\left\langle e\left|, \sigma_{+}=\right| e\right\rangle\langle g|$, and $\sigma_{z}=|e\rangle\langle e|-| g\rangle\langle g|$.

\section{A.2 Dispersive transformation}

We start from the Jaynes-Cummings Hamiltonian in Eq. (2.71), repeated here for convenience:

$$
H_{\mathrm{JC}}=\omega_{r} a^{\dagger} a+\frac{\omega_{a}}{2} \sigma_{z}+g\left(a \sigma_{+}+a^{\dagger} \sigma_{-}\right) .
$$

In the dispersive regime, $|\lambda|=|g / \Delta|=\left|g /\left(\omega_{\mathrm{a}}-\omega_{\mathrm{r}}\right)\right| \ll 1$, we then apply to this Hamiltonian the unitary transformation

$$
U_{\text {disp }}=\exp \left[\lambda\left(a \sigma_{+}-a^{\dagger} \sigma_{-}\right)\right] .
$$

The Baker-Hausdorff lemma, Eq. (A.5), gives

$$
\begin{aligned}
U_{\mathrm{disp}} a U_{\mathrm{disp}}^{\dagger}= & a+\lambda\left[a \sigma_{+}-a^{\dagger} \sigma_{-}, a\right] \\
& +\frac{\lambda^{2}}{2}\left[a \sigma_{+}-a^{\dagger} \sigma_{-},\left[a \sigma_{+}-a^{\dagger} \sigma_{-}, a\right]\right]+\mathcal{O}\left(\lambda^{3}\right) \\
= & a+\lambda \sigma_{-}+\frac{\lambda^{2}}{2}\left[a \sigma_{+}-a^{\dagger} \sigma_{-}, \sigma_{-}\right]+\mathcal{O}\left(\lambda^{3}\right) \\
= & a+\lambda \sigma_{-}+\frac{\lambda^{2}}{2} a \sigma_{z}+\mathcal{O}\left(\lambda^{3}\right),
\end{aligned}
$$


where we also used Eqs. (A.6) and (A.9). This also means that

$$
U_{\mathrm{disp}} a^{\dagger} U_{\mathrm{disp}}^{\dagger}=\left(U_{\mathrm{disp}} a U_{\mathrm{disp}}^{\dagger}\right)^{\dagger}=a^{\dagger}+\lambda \sigma_{+}+\frac{\lambda^{2}}{2} a^{\dagger} \sigma_{z}+\mathcal{O}\left(\lambda^{3}\right) \cdot(\mathrm{A}
$$

In a similar fashion, we calculate

$$
\begin{aligned}
U_{\mathrm{disp}} \sigma_{z} U_{\mathrm{disp}}^{\dagger}= & \sigma_{z}+\lambda\left[a \sigma_{+}-a^{\dagger} \sigma_{-}, \sigma_{z}\right] \\
& +\frac{\lambda^{2}}{2}\left[a \sigma_{+}-a^{\dagger} \sigma_{-},\left[a \sigma_{+}-a^{\dagger} \sigma_{-}, \sigma_{z}\right]\right]+\mathcal{O}\left(\lambda^{3}\right) \\
= & \sigma_{z}-2 \lambda\left(a \sigma_{+}+a^{\dagger} \sigma_{-}\right) \\
& -\lambda^{2}\left[a \sigma_{+}-a^{\dagger} \sigma_{-}, a \sigma_{+}+a^{\dagger} \sigma_{-}\right]+\mathcal{O}\left(\lambda^{3}\right) \\
= & \sigma_{z}-2 \lambda\left(a \sigma_{+}+a^{\dagger} \sigma_{-}\right)-\lambda^{2} \sigma_{z}\left(1+2 a^{\dagger} a\right)+\mathcal{O}\left(\lambda^{3}\right),
\end{aligned}
$$

where we used $\sigma_{+} \sigma_{-}=\left(1+\sigma_{z}\right) / 2$ and discarded constant terms since they do not affect the dynamics if they are included in the Hamiltonian.

Finally, we also calculate

$$
U_{\mathrm{disp}} \sigma_{-} U_{\mathrm{disp}}^{\dagger}=\sigma_{-}+\lambda a \sigma_{z}+\mathcal{O}\left(\lambda^{2}\right)
$$

where we only need to include the first-order terms since $\sigma_{-}$only appears in the weak interaction term of the Hamiltonian.

Applying our results to the transformation of the full Hamiltonian yields

$$
\begin{aligned}
U_{\mathrm{disp}} H_{\mathrm{JC}} U_{\text {disp }}^{\dagger}= & \omega_{\mathrm{r}} a^{\dagger} a+\lambda \omega_{\mathrm{r}}\left(a \sigma_{+}+a^{\dagger} \sigma_{-}\right)+\lambda^{2} \omega_{\mathrm{r}} \sigma_{z}\left(a^{\dagger} a+\frac{1}{2}\right) \\
& +\frac{\omega_{\mathrm{a}}}{2} \sigma_{z}-\lambda \omega_{\mathrm{a}}\left(a \sigma_{+}+a^{\dagger} \sigma_{-}\right)-\lambda^{2} \omega_{\mathrm{r}} \sigma_{z}\left(a^{\dagger} a+\frac{1}{2}\right) \\
& +g\left(a \sigma_{+}+a^{\dagger} \sigma_{-}\right)+\lambda g \sigma_{z}\left(2 a^{\dagger} a+1\right)+g \mathcal{O}\left(\lambda^{2}\right) \\
= & \omega_{\mathrm{r}} a^{\dagger} a+\frac{\omega_{\mathrm{a}}}{2} \sigma_{z}+\lambda g \sigma_{z}\left(a^{\dagger} a+\frac{1}{2}\right)+\mathcal{O}\left(\lambda^{2}\right) .
\end{aligned}
$$

Introducing the notation $\chi=g^{2} / \Delta$, the transformed Hamiltonian can be written

$$
H_{\text {disp }}=\left(\omega_{r}+\chi \sigma_{z}\right) a^{\dagger} a+\frac{\omega_{0}+\chi}{2} \sigma_{z}
$$


which is Eq. (2.78).

It is worth noting that there is a slightly different route to this result [310]. Defining

$$
\begin{aligned}
H_{\mathrm{JC}} & =H_{0}+H_{\mathrm{I}}, \\
H_{0} & =\omega_{r} a^{\dagger} a+\frac{\omega_{a}}{2} \sigma_{z}, \\
H_{\mathrm{I}} & =g\left(a \sigma_{+}+a^{\dagger} \sigma_{-}\right),
\end{aligned}
$$

and

$$
S=\lambda\left(a \sigma_{+}-a^{\dagger} \sigma_{-}\right),
$$

it is easy to show that

$$
\left[S, H_{0}\right]=-H_{\mathrm{I}} .
$$

Combining this result with the Baker-Hausdorff lemma gives

$$
\begin{aligned}
\exp (S) H_{\mathrm{JC}} \exp (-S) & =H_{\mathrm{JC}}+\left[S, H_{\mathrm{JC}}\right]+\frac{1}{2 !}\left[S,\left[S, H_{\mathrm{JC}}\right]\right]+\ldots \\
& =H_{0}+H_{\mathrm{I}}+\left[S, H_{\mathrm{I}}\right]-H_{\mathrm{I}}+\frac{1}{2}\left[S,-H_{\mathrm{I}}\right]+\ldots \\
& =H_{0}+\frac{1}{2}\left[S, H_{\mathrm{I}}\right]+\ldots
\end{aligned}
$$

where the remaining terms are of second order or higher in $S$. Using the final formula in Eq. (A.25) also gives the dispersive Hamiltonian in Eq. (A.19), but it does not give any information about how individual operators transform under $U_{\text {disp }}$, which can be useful in some contexts.

\section{A.3 Rotating frame}

As an example of a transformation that takes us to a rotating frame, we will consider transforming the Rabi Hamiltonian, Eq. (2.70),

$$
H_{\mathrm{Rabi}}=\omega_{r} a^{\dagger} a+\frac{\omega_{a}}{2} \sigma_{z}+g \sigma_{x}\left(a+a^{\dagger}\right),
$$

by applying

$$
U_{\text {rot }}=\exp \left(i \omega_{\mathrm{r}} t a^{\dagger} a+i \frac{\omega_{\mathrm{a}}}{2} t \sigma_{z}\right) .
$$

This will clarify the time dependence of the coupling terms and show when the RWA is valid. 
Since this transformation involves time, we must include the second term of Eq. (A.4), which becomes

$$
i \dot{U}_{\mathrm{rot}} U_{\mathrm{rot}}^{\dagger}=i\left(i \omega_{\mathrm{r}} a^{\dagger} a+i \frac{\omega_{\mathrm{a}}}{2} \sigma_{z}\right) U_{\mathrm{rot}} U_{\mathrm{rot}}^{\dagger}=-\omega_{\mathrm{r}} a^{\dagger} a-\frac{\omega_{\mathrm{a}}}{2} \sigma_{z} .
$$

Clearly, the first two terms in Eq. (A.26) commute with $U_{\text {rot }}$, so we only need to find the transformations for the third term. Eqs. (A.5) and (A.10) give

$$
\begin{aligned}
U_{\mathrm{rot}} \sigma_{-} U_{\mathrm{rot}}^{\dagger} & =\sigma_{-}+i \frac{\omega_{\mathrm{a}}}{2} t\left[\sigma_{z}, \sigma_{-}\right]+\frac{1}{2 !}\left(i \frac{\omega_{\mathrm{a}}}{2} t\right)\left[\sigma_{z},\left[\sigma_{z}, \sigma_{-}\right]\right]+\ldots \\
& =\sigma_{-}\left(1-i \omega_{\mathrm{a}} t+\frac{1}{2 !}\left(-i \omega_{\mathrm{a}} t\right)^{2}+\ldots\right)=\sigma_{-} e^{-i \omega_{\mathrm{a}} t},(\mathrm{~A}
\end{aligned}
$$

which also leads to

$$
U_{\mathrm{rot}} \sigma_{+} U_{\mathrm{rot}}^{\dagger}=\left(U_{\mathrm{rot}} \sigma_{-} U_{\mathrm{rot}}^{\dagger}\right)^{\dagger}=\sigma_{+} e^{i \omega_{\mathrm{a}} t} .
$$

In the same way, Eqs. (A.5) and (A.7) give

$$
\begin{aligned}
U_{\mathrm{rot}} a U_{\text {rot }}^{\dagger} & =a e^{-i \omega_{\mathrm{r}} t}, \\
U_{\mathrm{rot}} a^{\dagger} U_{\text {rot }}^{\dagger} & =a^{\dagger} e^{i \omega_{\mathrm{r}} t} .
\end{aligned}
$$

Combining all these results, we arrive at the transformed Hamiltonian

$$
\begin{aligned}
H_{\mathrm{rot}}= & U_{\mathrm{rot}} H_{\mathrm{Rabi}} U_{\text {rot }}^{\dagger}+i \dot{U}_{\mathrm{rot}} U_{\text {rot }}^{\dagger} \\
= & g\left(\sigma_{-} e^{-i \omega_{\mathrm{a}} t}+\sigma_{+} e^{i \omega_{\mathrm{a}} t}\right)\left(a e^{-i \omega_{\mathrm{r}} t}+a^{\dagger} e^{i \omega_{\mathrm{r}} t}\right) \\
= & g\left(a \sigma_{+} e^{i\left(\omega_{\mathrm{a}}-\omega_{\mathrm{r}}\right) t}+a^{\dagger} \sigma_{-} e^{i\left(\omega_{\mathrm{r}}-\omega_{\mathrm{a}}\right) t}\right. \\
& \left.+a \sigma_{-} e^{-i\left(\omega_{\mathrm{a}}+\omega_{\mathrm{r}}\right) t}+a^{\dagger} \sigma_{+} e^{i\left(\omega_{\mathrm{a}}+\omega_{\mathrm{r}}\right) t}\right) .
\end{aligned}
$$

The last two terms will always oscillate rapidly and can be discarded in the RWA provided that $g$ is small compared to $\omega_{\mathrm{a}}+\omega_{\mathrm{r}}$. 



\section{Appendix B}

\section{Deriving the master equation}

In this appendix, we present the full master equation derivation sketched in Sec. 3.1. Beginning from the Hamiltonian in Eqs. (3.4)-(3.7), repeated here,

$$
\begin{aligned}
H & =H_{\text {atom }}+H_{\text {bath }}+H_{\text {int }}, \\
H_{\text {atom }} & =\frac{\omega_{\mathrm{a}}}{2} \sigma_{z}, \\
H_{\text {bath }} & =\sum_{j} \omega_{j} b_{j}^{\dagger} b_{j}, \\
H_{\text {int }} & =\sum_{j} g_{j}\left(b_{j}+b_{j}^{\dagger}\right)\left(\sigma_{-}+\sigma_{+}\right),
\end{aligned}
$$

it was shown in Sec. 3.1 that the Born and Markov approximations lead to the density matrix equation

$$
\dot{\tilde{\rho}}(t)=-\int_{0}^{t} \mathrm{~d} \tau \operatorname{tr}_{\text {bath }}\left(\left[\tilde{H}_{\text {int }}(t),\left[\tilde{H}_{\text {int }}(\tau), \tilde{\rho}(t) \tilde{\rho}_{\text {bath }}\right]\right]\right),
$$

where $\tilde{\rho}(t)$ is the atom density matrix in the interaction picture. From the transformations in Appendix A, we get that the interaction Hamiltonian in the interaction picture is

$$
\tilde{H}_{\text {int }}(t)=\sum_{j} g_{j}\left(b_{j} e^{-i \omega_{j} t}+b_{j}^{\dagger} e^{i \omega_{j} t}\right)\left(\sigma_{-} e^{-i \omega_{\mathrm{a}} t}+\sigma_{+} e^{i \omega_{\mathrm{a}} t}\right) .
$$


We will now proceed with the gritty details of the derivation that leads to Eq. (3.13). It is convenient to define operators

$$
\begin{aligned}
& s(t)=\sigma_{-} e^{-i \omega_{\mathrm{a}} t} \\
& b(t)=\sum_{j} g_{j} b_{j} e^{-i \omega_{j} t},
\end{aligned}
$$

such that the interaction Hamiltonian can be written

$$
\tilde{H}_{\mathrm{int}}(t)=\left(b(t)+b^{\dagger}(t)\right)\left(s(t)+s^{\dagger}(t)\right) .
$$

When taking the trace in Eq. (B.5), the only nonzero terms will be

$$
\begin{aligned}
\operatorname{tr}_{\text {bath }}\left(b(t) b^{\dagger}(\tau) \tilde{\rho}_{\text {bath }}\right) & =\left\langle\sum_{j} g_{j} b_{j} e^{-i \omega_{j} t} \sum_{k} g_{k} b_{k}^{\dagger} e^{i \omega_{k} \tau}\right\rangle_{\text {bath }} \\
& =\sum_{j, k} g_{j} g_{k} e^{i\left(\omega_{k} \tau-\omega_{j} t\right)} \delta_{j k} \\
& =\sum_{j} g_{j}^{2} e^{-i \omega_{j}(t-\tau)}
\end{aligned}
$$

since $\left[b_{j}, b_{k}^{\dagger}\right]=\delta_{j k}$ and we assume negligible temperature, i.e., $\left\langle b_{j}^{\dagger} b_{j}\right\rangle_{\text {bath }}=$ 0. With this, Eq. (B.5) becomes

$$
\begin{aligned}
\dot{\tilde{\rho}}(t)= & -\int_{0}^{t} \mathrm{~d} \tau \sum_{j} g_{j}^{2}\left[e^{-i \omega_{j}(t-\tau)}\left(s(t) s^{\dagger}(\tau) \tilde{\rho}(t)+s^{\dagger}(t) s(\tau) \tilde{\rho}(t)\right)\right. \\
& -e^{i \omega_{j}(t-\tau)}\left(s(t) \tilde{\rho}(t) s^{\dagger}(\tau)+s^{\dagger}(t) \tilde{\rho}(t) s(\tau)\right) \\
& -e^{-i \omega_{j}(t-\tau)}\left(s(\tau) \tilde{\rho}(t) s^{\dagger}(t)+s^{\dagger}(\tau) \tilde{\rho}(t) s(t)\right) \\
& \left.+e^{i \omega_{j}(t-\tau)}\left(\tilde{\rho}(t) s(\tau) s^{\dagger}(t)+\tilde{\rho}(t) s^{\dagger}(\tau) s(t)\right)\right]
\end{aligned}
$$

where we have now also made the RWA. If we had made the RWA at the start in the Hamiltonian, half of the terms that now remain would be missing. This would not affect the final result for the relaxation rate, but it would have an impact on the Lamb shift [257, 258].

We now replace the sum over bath modes with an integral, denoting the density of states by $J(\omega)$. We also change variables in the time integral to $t^{\prime}=t-\tau$ and extend the upper integration limit there to $\infty$, which is justified since we have made the approximation that the bath correlations 
decay much faster than the timescale for the evolution of our system, the atom. Reverting back from our definitions of $s(t)$ and $b(t)$, we arrive at

$$
\begin{aligned}
\dot{\tilde{\rho}}(t)= & \int_{0}^{\infty} \mathrm{d} \omega J(\omega) g^{2}(\omega) \int_{0}^{\infty} \mathrm{d} t^{\prime}\left[e^{-i\left(-\omega+\omega_{\mathrm{a}}\right) t^{\prime}}\left(\sigma_{-} \tilde{\rho}(t) \sigma_{+}-\tilde{\rho}(t) \sigma_{+} \sigma_{-}\right)\right. \\
& +e^{-i\left(-\omega-\omega_{\mathrm{a}}\right) t^{\prime}}\left(\sigma_{+} \tilde{\rho}(t) \sigma_{-}-\tilde{\rho}(t) \sigma_{-} \sigma_{+}\right) \\
& +e^{-i\left(\omega+\omega_{\mathrm{a}}\right) t^{\prime}}\left(\sigma_{+} \tilde{\rho}(t) \sigma_{-}-\sigma_{-} \sigma_{+} \tilde{\rho}(t)\right) \\
& \left.+e^{-i\left(\omega-\omega_{\mathrm{a}}\right) t^{\prime}}\left(\sigma_{-} \tilde{\rho}(t) \sigma_{+}-\sigma_{+} \sigma_{-} \tilde{\rho}(t)\right)\right]
\end{aligned}
$$

Applying the identity

$$
\int_{0}^{\infty} \mathrm{d} t e^{-i \omega t}=\pi \delta(\omega)-i \mathcal{P}\left(\frac{1}{\omega}\right)
$$

to Eq. (B.12) yields, after a little algebra,

$$
\begin{aligned}
\dot{\tilde{\rho}}(t)= & \pi J\left(\omega_{\mathrm{a}}\right) g^{2}\left(\omega_{\mathrm{a}}\right)\left(\sigma_{-} \tilde{\rho}(t) \sigma_{+}-\tilde{\rho}(t) \sigma_{+} \sigma_{-}+\sigma_{-} \tilde{\rho}(t) \sigma_{+}-\sigma_{+} \sigma_{-} \tilde{\rho}(t)\right) \\
& +i \mathcal{P} \int_{0}^{\infty} \mathrm{d} \omega \frac{J(\omega) g^{2}(\omega)}{\omega-\omega_{\mathrm{a}}} \\
& \times\left(\sigma_{-} \tilde{\rho}(t) \sigma_{+}-\tilde{\rho}(t) \sigma_{+} \sigma_{-}-\sigma_{-} \tilde{\rho}(t) \sigma_{+}+\sigma_{+} \sigma_{-} \tilde{\rho}(t)\right) \\
& +i \mathcal{P} \int_{0}^{\infty} \mathrm{d} \omega \frac{J(\omega) g^{2}(\omega)}{\omega+\omega_{\mathrm{a}}} \\
& \times\left(\sigma_{+} \tilde{\rho}(t) \sigma_{-}-\tilde{\rho}(t) \sigma_{-} \sigma_{+}-\sigma_{+} \tilde{\rho}(t) \sigma_{-}+\sigma_{-} \sigma_{+} \tilde{\rho}(t)\right)
\end{aligned}
$$

Clearing up terms and using the notation $\mathcal{D}[X] \rho=X \rho X^{\dagger}-\frac{1}{2} X^{\dagger} X \rho-$ $\frac{1}{2} \rho X^{\dagger} X$ gives

$$
\begin{aligned}
\dot{\tilde{\rho}}(t)= & 2 \pi J\left(\omega_{\mathrm{a}}\right) g^{2}\left(\omega_{\mathrm{a}}\right) \mathcal{D}\left[\sigma_{-}\right] \tilde{\rho}(t) \\
& +i \mathcal{P} \int_{0}^{\infty} \mathrm{d} \omega \frac{J(\omega) g^{2}(\omega)}{\omega-\omega_{\mathrm{a}}}\left[\sigma_{+} \sigma_{-}, \tilde{\rho}(t)\right] \\
& +i \mathcal{P} \int_{0}^{\infty} \mathrm{d} \omega \frac{J(\omega) g^{2}(\omega)}{\omega+\omega_{\mathrm{a}}}\left[\sigma_{-} \sigma_{+}, \tilde{\rho}(t)\right] .
\end{aligned}
$$

Using

$$
\begin{aligned}
& \sigma_{+} \sigma_{-}=\frac{1+\sigma_{z}}{2}, \\
& \sigma_{-} \sigma_{+}=\frac{1-\sigma_{z}}{2},
\end{aligned}
$$


we finally get

$$
\begin{aligned}
\dot{\tilde{\rho}}(t)= & 2 \pi J\left(\omega_{\mathrm{a}}\right) g^{2}\left(\omega_{\mathrm{a}}\right) \mathcal{D}\left[\sigma_{-}\right] \tilde{\rho}(t) \\
& -i\left[\frac{\sigma_{z}}{2}, \tilde{\rho}(t)\right] \mathcal{P} \int_{0}^{\infty} \mathrm{d} \omega J(\omega) g^{2}(\omega)\left(\frac{1}{\omega+\omega_{\mathrm{a}}}-\frac{1}{\omega-\omega_{\mathrm{a}}}\right) .
\end{aligned}
$$

Transforming back out of the interaction picture, the only effect is to remove the tilde from $\tilde{\rho}(t)$ and bring back the term with $\frac{\omega_{\mathrm{a}}}{2} \sigma_{z}$. We thus arrive at Eq. (3.13). 


\section{Bibliography}

[1] E. Schrödinger, "Are There Quantum Jumps? Part II", The British Journal for the Philosophy of Science 3, 233 (1952).

[2] Royal Swedish Academy of Sciences, The Nobel Prize in Physics 2012 - Advanced Information, (2012) www . nobelprize.org/nobel_ prizes / physics / laureates / 2012 / advanced . html (visited on 08/22/2014).

[3] S. Haroche, "Nobel Lecture: Controlling photons in a box and exploring the quantum to classical boundary", Reviews of Modern Physics 85, 1083 (2013).

[4] D. J. Wineland, "Nobel Lecture: Superposition, entanglement, and raising Schrödinger's cat", Reviews of Modern Physics 85, 1103 (2013).

[5] P. Goy, J. M. Raimond, M. Gross, and S. Haroche, "Observation of Cavity-Enhanced Single-Atom Spontaneous Emission", Physical Review Letters 50, 1903 (1983).

[6] W. Jhe, A. Anderson, E. A. Hinds, D. Meschede, L. Moi, and S. Haroche, "Suppression of spontaneous decay at optical frequencies: Test of vacuum-field anisotropy in confined space", Physical Review Letters 58, 666 (1987).

[7] M. Brune, J. M. Raimond, P. Goy, L. Davidovich, and S. Haroche, "Realization of a two-photon maser oscillator", Physical Review Letters 59, 1899 (1987).

[8] C. Guerlin, J. Bernu, S. Deléglise, C. Sayrin, S. Gleyzes, S. Kuhr, M. Brune, J. M. Raimond, and S. Haroche, "Progressive field-state collapse and quantum non-demolition photon counting", Nature 448, 889 (2007). 
[9] D. J. Wineland, R. E. Drullinger, and F. L. Walls, "RadiationPressure Cooling of Bound Resonant Absorbers", Physical Review Letters 40, 1639 (1978).

[10] D. J. Wineland and W. M. Itano, "Spectroscopy of a single $\mathrm{Mg}^{+}$ ion", Physics Letters A 82, 75 (1981).

[11] J. C. Bergquist, R. G. Hulet, W. M. Itano, and D. J. Wineland, "Observation of Quantum Jumps in a Single Atom", Physical Review Letters 57, 1699 (1986).

[12] C. Monroe, D. M. Meekhof, B. E. King, S. R. Jefferts, W. M. Itano, D. J. Wineland, and P. Gould, "Resolved-Sideband Raman Cooling of a Bound Atom to the 3D Zero-Point Energy", Physical Review Letters 75, 4011 (1995).

[13] C. Monroe, D. M. Meekhof, B. E. King, W. M. Itano, and D. J. Wineland, "Demonstration of a Fundamental Quantum Logic Gate", Physical Review Letters 75, 4714 (1995).

[14] M. Brune, E. Hagley, J. Dreyer, X. Maître, A. Maali, C. Wunderlich, J. M. Raimond, and S. Haroche, "Observing the Progressive Decoherence of the "Meter" in a Quantum Measurement", Physical Review Letters 77, 4887 (1996).

[15] C. R. Monroe, D. M. Meekhof, B. E. King, and D. J. Wineland, "A "Schrödinger Cat" Superposition State of an Atom", Science 272, 1131 (1996).

[16] T. D. Ladd, F. Jelezko, R. Laflamme, Y. Nakamura, C. Monroe, and J. L. O'Brien, "Quantum computers", Nature 464, 45 (2010).

[17] I. Buluta, S. Ashhab, and F. Nori, "Natural and artificial atoms for quantum computation", Reports on Progress in Physics 74, 104401 (2011).

[18] R. Miller, T. E. Northup, K. M. Birnbaum, A. Boca, A. D. Boozer, and H. J. Kimble, "Trapped atoms in cavity QED: coupling quantized light and matter", Journal of Physics B: Atomic, Molecular and Optical Physics 38, S551 (2005).

[19] H. Walther, B. T. H. Varcoe, B.-G. Englert, and T. Becker, "Cavity quantum electrodynamics", Reports on Progress in Physics 69, 1325 (2006). 
[20] R. Hanson, L. P. Kouwenhoven, J. Petta, S. Tarucha, and L. M. K. Vandersypen, "Spins in few-electron quantum dots", Reviews of Modern Physics 79, 1217 (2007).

[21] M. V. G. Dutt, L. Childress, L. Jiang, E. Togan, J. Maze, F. Jelezko, A. S. Zibrov, P. R. Hemmer, and M. D. Lukin, "Quantum Register Based on Individual Electronic and Nuclear Spin Qubits in Diamond", Science 316, 1312 (2007).

[22] H. de Riedmatten, M. Afzelius, M. U. Staudt, C. Simon, and N. Gisin, "A solid-state light-matter interface at the single-photon level", Nature 456, 773 (2008).

[23] A. Blais, R.-S. Huang, A. Wallraff, S. M. Girvin, and R. J. Schoelkopf, "Cavity quantum electrodynamics for superconducting electrical circuits: An architecture for quantum computation", Physical Review A 69, 062320 (2004).

[24] A. Wallraff, D. I. Schuster, A. Blais, L. Frunzio, R.-S. Huang, J. Majer, S. Kumar, S. M. Girvin, and R. J. Schoelkopf, "Strong coupling of a single photon to a superconducting qubit using circuit quantum electrodynamics", Nature 431, 162 (2004).

[25] J. Q. You and F. Nori, "Superconducting Circuits and Quantum Information", Physics Today 58, 42 (2005).

[26] R. J. Schoelkopf and S. M. Girvin, "Wiring up quantum systems", Nature 451, 664 (2008).

[27] J. Clarke and F. K. Wilhelm, "Superconducting quantum bits", Nature 453, 1031 (2008).

[28] M. H. Devoret and R. J. Schoelkopf, "Superconducting Circuits for Quantum Information: An Outlook", Science 339, 1169 (2013).

[29] B. D. Josephson, "Possible new effects in superconductive tunnelling", Physics Letters 1, 251 (1962).

[30] J. R. Waldram, Superconductivity of metals and cuprates (IOP Publishing Ltd, 1996).

[31] N. Matsuda, R. Shimizu, Y. Mitsumori, H. Kosaka, and K. Edamatsu, "Observation of optical-fibre Kerr nonlinearity at the single-photon level", Nature Photonics 3, 95 (2009). 
[32] V. Venkataraman, K. Saha, and A. L. Gaeta, "Phase modulation at the few-photon level for weak-nonlinearity-based quantum computing", Nature Photonics 7, 138 (2012).

[33] C. Perrella, P. S. Light, J. D. Anstie, F. Benabid, T. M. Stace, A. G. White, and A. N. Luiten, "High-efficiency cross-phase modulation in a gas-filled waveguide", Physical Review A 88, 013819 (2013).

[34] J. Eschner, C. Raab, F. Schmidt-Kaler, and R. Blatt, "Light interference from single atoms and their mirror images", Nature 413, 495 (2001).

[35] O. Astafiev, A. M. Zagoskin, A. A. Abdumalikov Jr., Y. A. Pashkin, T. Yamamoto, K. Inomata, Y. Nakamura, and J. S. Tsai, "Resonance Fluorescence of a Single Artificial Atom", Science 327, 840 (2010).

[36] A. A. Abdumalikov Jr., O. Astafiev, A. M. Zagoskin, Y. A. Pashkin, Y. Nakamura, and J. S. Tsai, "Electromagnetically Induced Transparency on a Single Artificial Atom", Physical Review Letters 104, 193601 (2010).

[37] I.-C. Hoi, C. M. Wilson, G. Johansson, T. Palomaki, B. Peropadre, and P. Delsing, "Demonstration of a Single-Photon Router in the Microwave Regime", Physical Review Letters 107, 073601 (2011).

[38] I.-C. Hoi, T. Palomaki, J. Lindkvist, G. Johansson, P. Delsing, and C. M. Wilson, "Generation of Nonclassical Microwave States Using an Artificial Atom in 1D Open Space", Physical Review Letters 108, 263601 (2012).

[39] I.-C. Hoi, C. M. Wilson, G. Johansson, J. Lindkvist, B. Peropadre, T. Palomaki, and P. Delsing, "Microwave quantum optics with an artificial atom in one-dimensional open space", New Journal of Physics 15, 025011 (2013).

[40] I.-C. Hoi, "Quantum Optics with Propagating Microwaves in Superconducting Circuits", $\mathrm{PhD}$ thesis (Chalmers University of Technology, Gothenburg, 2013).

[41] A. F. van Loo, A. Fedorov, K. Lalumière, B. C. Sanders, A. Blais, and A. Wallraff, "Photon-Mediated Interactions Between Distant Artificial Atoms", Science 342, 1494 (2013). 
[42] R. G. DeVoe and R. G. Brewer, "Observation of Superradiant and Subradiant Spontaneous Emission of Two Trapped Ions", Physical Review Letters 76, 2049 (1996).

[43] I. Gerhardt, G. Wrigge, P. Bushev, G. Zumofen, M. Agio, R. Pfab, and V. Sandoghdar, "Strong Extinction of a Laser Beam by a Single Molecule", Physical Review Letters 98, 033601 (2007).

[44] G. Wrigge, I. Gerhardt, J. Hwang, G. Zumofen, and V. Sandoghdar, "Efficient coupling of photons to a single molecule and the observation of its resonance fluorescence", Nature Physics 4, 60 (2008).

[45] M. K. Tey, Z. Chen, S. A. Aljunid, B. Chng, F. Huber, G. Maslennikov, and C. Kurtsiefer, "Strong interaction between light and a single trapped atom without the need for a cavity", Nature Physics 4, 924 (2008).

[46] J. Hwang, M. Pototschnig, R. Lettow, G. Zumofen, A. Renn, S. Götzinger, and V. Sandoghdar, "A single-molecule optical transistor", Nature 460, 76 (2009).

[47] A. N. Vamivakas, M. Atatüre, J. Dreiser, S. T. Yilmaz, A. Badolato, A. K. Swan, B. B. Goldberg, A. Imamoğlu, and M. S. Unlü, "Strong Extinction of a Far-Field Laser Beam by a Single Quantum Dot", Nano Letters 7, 2892 (2007).

[48] A. Muller, E. B. Flagg, P. Bianucci, X. Y. Wang, D. G. Deppe, W. Ma, J. Zhang, G. J. Salamo, M. Xiao, and C. K. Shih, "Resonance Fluorescence from a Coherently Driven Semiconductor Quantum Dot in a Cavity", Physical Review Letters 99, 187402 (2007).

[49] R. H. Hadfield, "Single-photon detectors for optical quantum information applications", Nature Photonics 3, 696 (2009).

[50] M. D. Eisaman, J. Fan, A. Migdall, and S. V. Polyakov, "Singlephoton sources and detectors", Review of Scientific Instruments 82, 071101 (2011).

[51] F. Helmer, M. Mariantoni, E. Solano, and F. Marquardt, "Quantum nondemolition photon detection in circuit QED and the quantum Zeno effect", Physical Review A 79, 052115 (2009).

[52] G. Romero, J. J. Garcia-Ripoll, and E. Solano, "Microwave Photon Detector in Circuit QED", Physical Review Letters 102, 173602 (2009). 
[53] G. Romero, J. J. García-Ripoll, and E. Solano, "Photodetection of propagating quantum microwaves in circuit QED", Physica Scripta T137, 014004 (2009).

[54] B. Peropadre, G. Romero, G. Johansson, C. M. Wilson, E. Solano, and J. J. Garcia-Ripoll, "Approaching perfect microwave photodetection in circuit QED", Physical Review A 84, 063834 (2011).

[55] Y.-F. Chen, D. Hover, S. Sendelbach, L. Maurer, S. T. Merkel, E. J. Pritchett, F. K. Wilhelm, and R. McDermott, "Microwave Photon Counter Based on Josephson Junctions", Physical Review Letters 107, 217401 (2011).

[56] N. Imoto, H. A. Haus, and Y. Yamamoto, "Quantum nondemolition measurement of the photon number via the optical Kerr effect", Physical Review A 32, 2287 (1985).

[57] W. J. Munro, K. Nemoto, R. G. Beausoleil, and T. P. Spiller, "Highefficiency quantum-nondemolition single-photon-number-resolving detector", Physical Review A 71, 033819 (2005).

[58] T. J. Kippenberg and K. J. Vahala, "Cavity Optomechanics: BackAction at the Mesoscale", Science 321, 1172 (2008).

[59] C. Genesa, A. Marib, D. Vitalic, and P. Tombesi, "Quantum Effects in Optomechanical Systems", Advances In Atomic, Molecular, and Optical Physics 57, 33 (2009).

[60] I. Favero and K. Karrai, "Optomechanics of deformable optical cavities", Nature Photonics 3, 201 (2009).

[61] F. Marquardt and S. M. Girvin, "Optomechanics", Physics 2, 40 (2009).

[62] M. Poot and H. S. J. van der Zant, "Mechanical systems in the quantum regime", Physics Reports 511, 273 (2012).

[63] M. Aspelmeyer, P. Meystre, and K. Schwab, "Quantum optomechanics", Physics Today 65, 29 (2012).

[64] M. Aspelmeyer, T. J. Kippenberg, and F. Marquardt, "Cavity optomechanics", arXiv:1303.0733, to appear in Reviews of Modern Physics (2013). 
[65] F. Marquardt, "Quantum optomechanics", in Quantum Machines: Measurement and Control of Engineered Quantum Systems (Les Houches Session XCVI), edited by M. H. Devoret, B. Huard, R. J. Schoelkopf, and L. F. Cugliandolo (Oxford University Press, 2014).

[66] S. Gigan, H. R. Böhm, M. Paternostro, F. Blaser, G. Langer, J. B. Hertzberg, K. C. Schwab, D. Bäuerle, M. Aspelmeyer, and A. Zeilinger, "Self-cooling of a micromirror by radiation pressure", Nature 444, 67 (2006).

[67] O. Arcizet, P.-F. Cohadon, T. Briant, M. Pinard, and A. Heidmann, "Radiation-pressure cooling and optomechanical instability of a micromirror", Nature 444, 71 (2006).

[68] A. Schliesser, P. Del'Haye, N. Nooshi, K. J. Vahala, and T. J. Kippenberg, "Radiation Pressure Cooling of a Micromechanical Oscillator Using Dynamical Backaction", Physical Review Letters 97, 243905 (2006).

[69] J. D. Thompson, B. M. Zwickl, A. M. Jayich, F. Marquardt, S. M. Girvin, and J. G. E. Harris, "Strong dispersive coupling of a high-finesse cavity to a micromechanical membrane", Nature 452, 72 (2008).

[70] G. Anetsberger, O. Arcizet, Q. P. Unterreithmeier, R. Rivière, A. Schliesser, E. M. Weig, J. P. Kotthaus, and T. J. Kippenberg, "Nearfield cavity optomechanics with nanomechanical oscillators", Nature Physics 5, 909 (2009).

[71] K. W. Murch, K. L. Moore, S. Gupta, and D. M. Stamper-Kurn, "Near-field cavity optomechanics with nanomechanical oscillators", Nature Physics 4, 561 (2008).

[72] F. Brennecke, S. Ritter, T. Donner, and T. Esslinger, "Cavity Optomechanics with a Bose-Einstein Condensate", Science 322, 235 (2008).

[73] M. Eichenfield, R. Camacho, J. Chan, K. J. Vahala, and O. Painter, "A picogram- and nanometre-scale photonic-crystal optomechanical cavity", Nature 459, 550 (2009).

[74] M. Blencowe, "Quantum electromechanical systems", Physics Reports 395, 159 (2003). 
[75] C. A. Regal, J. D. Teufel, and K. W. Lehnert, "Measuring nanomechanical motion with a microwave cavity interferometer", Nature Physics 4, 555 (2008).

[76] A. D. O'Connell, M. Hofheinz, M. Ansmann, R. C. Bialczak, M. Lenander, E. Lucero, M. Neeley, D. Sank, H. Wang, M. Weides, J. Wenner, J. M. Martinis, and A. N. Cleland, "Quantum ground state and single-phonon control of a mechanical resonator", Nature 464, 697 (2010).

[77] J. D. Teufel, D. Li, M. S. Allman, K. Cicak, A. J. Sirois, J. D. Whittaker, and R. W. Simmonds, "Circuit cavity electromechanics in the strong-coupling regime", Nature 471, 204 (2011).

[78] K. W. Lehnert, "Micromechanics and superconducting circuits", in Quantum Machines: Measurement and Control of Engineered Quantum Systems (Les Houches Session XCVI), edited by M. H. Devoret, B. Huard, R. J. Schoelkopf, and L. F. Cugliandolo (Oxford University Press, 2014).

[79] J. Chan, T. P. M. Alegre, A. H. Safavi-Naeini, J. T. Hill, A. Krause, S. Gröblacher, M. Aspelmeyer, and O. Painter, "Laser cooling of a nanomechanical oscillator into its quantum ground state", Nature 478, 89 (2011).

[80] J. D. Teufel, T. Donner, D. Li, J. W. Harlow, M. S. Allman, K. Cicak, A. J. Sirois, J. D. Whittaker, K. W. Lehnert, and R. W. Simmonds, "Sideband cooling of micromechanical motion to the quantum ground state", Nature 475, 359 (2011).

[81] J. Johansson, G. Johansson, and F. Nori, "Optomechanical-like coupling between superconducting resonators", Physical Review A 90, 053833 (2014).

[82] R. C. Jaklevic, J. Lambe, A. H. Silver, and J. E. Mercereau, "Quantum Interference Effects in Josephson Tunneling", Physical Review Letters 12, 159 (1964).

[83] L. Tian, P. Rabl, R. Blatt, and P. Zoller, "Interfacing QuantumOptical and Solid-State Qubits", Physical Review Letters 92, 247902 (2004).

[84] L. Childress, A. S. Sørensen, and M. D. Lukin, "Mesoscopic cavity quantum electrodynamics with quantum dots", Physical Review A 69, 042302 (2004). 
[85] Y. Colombe, T. Steinmetz, G. Dubois, F. Linke, D. Hunger, and J. Reichel, "Strong atom-field coupling for Bose-Einstein condensates in an optical cavity on a chip", Nature 450, 272 (2007).

[86] M Wallquist, K Hammerer, P Rabl, M Lukin, and P Zoller, "Hybrid quantum devices and quantum engineering", Physica Scripta T137, 014001 (2009).

[87] J. Verdú, H. Zoubi, C. Koller, J. Majer, H. Ritsch, and J. Schmiedmayer, "Strong Magnetic Coupling of an Ultracold Gas to a Superconducting Waveguide Cavity", Physical Review Letters 103, 043603 (2009).

[88] T. Duty, "Towards superconductor-spin ensemble hybrid quantum systems", Physics 3, 80 (2010).

[89] Z.-L. Xiang, S. Ashhab, J. Q. You, and F. Nori, "Hybrid quantum circuits: Superconducting circuits interacting with other quantum systems", Reviews of Modern Physics 85, 623 (2013).

[90] G.-W. Deng, D. Wei, J. Johansson, M.-L. Zhang, S.-X. Li, H.-O. Li, G. Cao, M. Xiao, T. Tu, G.-C. Guo, H.-W. Jiang, F. Nori, and G.-P. Guo, "Circuit QED with a graphene double quantum dot and a reflection-line resonator", arXiv:1310.6118 (2013).

[91] Y. Tabuchi, S. Ishino, T. Ishikawa, R. Yamazaki, K. Usami, and Y. Nakamura, "Hybridizing Ferromagnetic Magnons and Microwave Photons in the Quantum Limit", Physical Review Letters 113, 083603 (2014).

[92] Y. Tabuchi, S. Ishino, A. Noguchi, T. Ishikawa, R. Yamazaki, K. Usami, and Y. Nakamura, "Coherent coupling between ferromagnetic magnon and superconducting qubit", arXiv:1410.3781 (2014).

[93] S. Datta, Surface Acoustic Wave Devices (Prentice Hall, 1986).

[94] D. Morgan, Surface Acoustic Wave Filters, 2nd ed. (Academic Press, 2007).

[95] C. K. Campbell, Surface Acoustic Wave Devices and their Signal Processing Applications (Academic Press, 1989).

[96] K. Hashimoto, Surface Acoustic Wave Devices in Telecommunications (Springer-Verlag, 2000). 
[97] P. D. Nation, J. R. Johansson, M. P. Blencowe, and F. Nori, "Stimulating uncertainty: Amplifying the quantum vacuum with superconducting circuits", Reviews of Modern Physics 84, 1 (2012).

[98] G. T. Moore, "Quantum Theory of the Electromagnetic Field in a Variable-Length One-Dimensional Cavity", Journal of Mathematical Physics 11, 2679 (1970).

[99] J. R. Johansson, G. Johansson, C. M. Wilson, and F. Nori, "Dynamical Casimir Effect in a Superconducting Coplanar Waveguide", Physical Review Letters 103, 147003 (2009).

[100] J. R. Johansson, G. Johansson, C. M. Wilson, and F. Nori, "Dynamical Casimir effect in superconducting microwave circuits", Physical Review A 82, 052509 (2010).

[101] C. M. Wilson, G. Johansson, A. Pourkabirian, M. Simoen, J. R. Johansson, T. Duty, F. Nori, and P. Delsing, "Observation of the dynamical Casimir effect in a superconducting circuit", Nature 479, 376 (2011).

[102] J. Lindkvist, C. Sabín, I. Fuentes, A. Dragan, I.-M. Svensson, P. Delsing, and G. Johansson, "Twin paradox with macroscopic clocks in superconducting circuits", Physical Review A 90, 052113 (2014).

[103] N. Friis, A. R. Lee, K. Truong, C. Sabín, E. Solano, G. Johansson, and I. Fuentes, "Relativistic Quantum Teleportation with Superconducting Circuits", Physical Review Letters 110, 113602 (2013).

[104] J. J. Sakurai, Modern Quantum Mechanics (Addison-Wesley Publishing Company, Inc., 1994).

[105] C. C. Gerry and P. L. Knight, Introductory Quantum Optics (Cambridge University Press, 2005).

[106] M. E. Peskin and D. V. Schroeder, An Introduction to Quantum Field Theory (Westview Press, 1995).

[107] H. B. G. Casimir, "On the attraction between two perfectly conducting plates", Proceedings of the Royal Netherlands Academy of Arts and Sciences 51, 793 (1948).

[108] M. J. Sparnaay, "Attractive Forces between Flat Plates", Nature 180, 334 (1957).

[109] S. W. Hawking, "Black hole explosions?", Nature 248, 30 (1974). 
[110] W. G. Unruh, "Notes on black-hole evaporation", Physical Review D 14, 870 (1976).

[111] W. E. Lamb and R. C. Retherford, "Fine Structure of the Hydrogen Atom by a Microwave Method", Physical Review 72, 241 (1947).

[112] H. A. Bethe, "The Electromagnetic Shift of Energy Levels", Physical Review 72, 339 (1947).

[113] A. Fragner, M. Göppl, J. M. Fink, M. Baur, R. Bianchetti, P. J. Leek, A. Blais, and A. Wallraff, "Resolving Vacuum Fluctuations in an Electrical Circuit by Measuring the Lamb Shift", Science 322, 1357 (2008).

[114] V. F. Weisskopf and E. Wigner, "Berechnung der natürlichen Linienbreite auf Grund der Diracschen Lichttheorie", Zeitschrift für Physik 63, 54 (1930).

[115] E. M. Purcell, "Spontaneous Emission Probabilities at Radio Frequencies", Physical Review 69, 681 (1946).

[116] C. W. Gardiner and P. Zoller, Quantum Noise, 3rd ed. (Springer, 2004).

[117] H. Nyquist, "Thermal Agitation of Electric Charge in Conductors", Physical Review 32, 110 (1928).

[118] H. B. Callen and T. A. Welton, "Irreversibility and Generalized Noise", Physical Review 83, 34 (1951).

[119] R. Kubo, "The fluctuation-dissipation theorem", Reports on Progress in Physics 29, 255 (1966).

[120] A. A. Clerk, M. H. Devoret, S. M. Girvin, F. Marquardt, and R. J. Schoelkopf, "Introduction to quantum noise, measurement, and amplification", Reviews of Modern Physics 82, 1155 (2010).

[121] R. P. Feynman, "Simulating Physics with Computers", International Journal of Theoretical Physics 21, 467 (1982).

[122] M. A. Nielsen and I. L. Chuang, Quantum computation and quantum information (Cambridge University Press, 2000).

[123] D. Deutsch, "Quantum Theory, the Church-Turing Principle and the Universal Quantum Computer", Proceedings of the Royal Society A: Mathematical, Physical and Engineering Sciences 400, 97 (1985). 
[124] P. W. Shor, "Algorithms for Quantum Computation: Discrete Logarithms and Factoring", in Proceedings of the 35th annual symposium on foundations of computer science, SFCS '94 (1994), pp. 124-134.

[125] L. K. Grover, "Quantum Mechanics Helps in Searching for a Needle in a Haystack", Physical Review Letters 79, 325 (1997).

[126] A. W. Harrow, A. Hassidim, and S. Lloyd, "Quantum Algorithm for Linear Systems of Equations", Physical Review Letters 103, 150502 (2009).

[127] P. Rebentrost, M. Mohseni, and S. Lloyd, "Quantum Support Vector Machine for Big Data Classification", Physical Review Letters 113, 130503 (2014).

[128] D. P. DiVincenzo, "The Physical Implementation of Quantum Computation", Fortschritte der Physik 48, 771 (2000).

[129] J. I. Cirac and P. Zoller, "Quantum Computations with Cold Trapped Ions", Physical Review Letters 74, 4091 (1995).

[130] N. A. Gershenfeld and I. L. Chuang, "Bulk Spin-Resonance Quantum Computation", Science 275, 350 (1997).

[131] D. Loss and D. P. DiVincenzo, "Quantum computation with quantum dots", Physical Review A 57, 120 (1998).

[132] E. Knill, R. Laflamme, and G. J. Milburn, "A scheme for efficient quantum computation with linear optics", Nature 409, 46 (2001).

[133] A. Blais, J. Gambetta, A. Wallraff, D. I. Schuster, S. M. Girvin, M. H. Devoret, and R. J. Schoelkopf, "Quantum-information processing with circuit quantum electrodynamics", Physical Review A 75, 032329 (2007).

[134] L. M. K. Vandersypen, M. Steffen, G. Breyta, C. S. Yannoni, M. H. Sherwood, and I. L. Chuang, "Experimental realization of Shor's quantum factoring algorithm using nuclear magnetic resonance", Nature 414, 883 (2001).

[135] H. Häffner, W. Hänsel, C. F. Roos, J. Benhelm, D. C. al kar, M. Chwalla, T. Körber, U. D. Rapol, M. Riebe, P. O. Schmidt, C. Becher, O. Gühne, W. Dür, and R. Blatt, "Scalable multiparticle entanglement of trapped ions", Nature 438, 643 (2005). 
[136] T. Monz, P. Schindler, J. T. Barreiro, M. Chwalla, D. Nigg, W. A. Coish, M. Harlander, W. Hänsel, M. Hennrich, and R. Blatt, "14-Qubit Entanglement: Creation and Coherence", Physical Review Letters 106, 130506 (2011).

[137] R. Barends et al., "Superconducting quantum circuits at the surface code threshold for fault tolerance", Nature 508, 500 (2014).

[138] M. W. Johnson et al., "Quantum annealing with manufactured spins", Nature 473, 194 (2011).

[139] N. G. Dickson et al., "Thermally assisted quantum annealing of a 16-qubit problem", Nature Communications 4, 1903 (2013).

[140] T. Lanting et al., "Entanglement in a Quantum Annealing Processor", Physical Review X 4, 021041 (2014).

[141] S. Boixo, T. F. Rønnow, S. V. Isakov, Z. Wang, D. Wecker, D. A. Lidar, J. M. Martinis, and M. Troyer, "Evidence for quantum annealing with more than one hundred qubits", Nature Physics 10, 218 (2014).

[142] T. F. Rønnow, Z. Wang, J. Job, S. Boixo, S. V. Isakov, D. Wecker, J. M. Martinis, D. A. Lidar, and M. Troyer, "Defining and detecting quantum speedup", Science 345, 420 (2014).

[143] S. W. Shin, G. Smith, J. A. Smolin, and U. Vazirani, "How "Quantum" is the D-Wave Machine?", arXiv:1401.7087v2 (2014).

[144] T. Albash, W. Vinci, A. Mishra, P. A. Warburton, and D. A. Lidar, "Distinguishing Classical and Quantum Models for the D-Wave Device", arXiv:1403.4228v3 (2014).

[145] I. Buluta and F. Nori, "Quantum Simulators", Science 326, 108 (2009).

[146] I. Kassal, J. D. Whitfield, A. Perdomo-Ortiz, M.-H. Yung, and A. Aspuru-Guzik, "Simulating Chemistry Using Quantum Computers", Annual Review of Physical Chemistry 62, 185 (2011).

[147] I. Bloch, J. Dalibard, and S. Nascimbène, "Quantum simulations with ultracold quantum gases", Nature Physics 8, 267 (2012).

[148] R. Blatt and C. F. Roos, "Quantum simulations with trapped ions", Nature Physics 8, 277 (2012).

[149] A. Aspuru-Guzik and P. Walther, "Photonic quantum simulators", Nature Physics 8, 285 (2012). 
[150] A. A. Houck, H. E. Türeci, and J. Koch, "On-chip quantum simulation with superconducting circuits", Nature Physics 8, 292 (2012).

[151] I. M. Georgescu, S. Ashhab, and F. Nori, "Quantum Simulation", Reviews of Modern Physics 86, 153 (2014).

[152] J. Raftery, D. Sadri, S. Schmidt, H. E. Türeci, and A. A. Houck, "Observation of a Dissipation-Induced Classical to Quantum Transition", Physical Review X 4, 031043 (2014).

[153] H. Paik, D. I. Schuster, L. S. Bishop, G. Kirchmair, G. Catelani, A. P. Sears, B. R. Johnson, M. J. Reagor, L. Frunzio, L. I. Glazman, S. M. Girvin, M. H. Devoret, and R. J. Schoelkopf, "Observation of High Coherence in Josephson Junction Qubits Measured in a ThreeDimensional Circuit QED Architecture", Physical Review Letters 107, 240501 (2011).

[154] C. Rigetti, J. M. Gambetta, S. Poletto, B. L. T. Plourde, J. M. Chow, A. D. Córcoles, J. A. Smolin, S. T. Merkel, J. R. Rozen, G. A. Keefe, M. B. Rothwell, M. B. Ketchen, and M. Steffen, "Superconducting qubit in a waveguide cavity with a coherence time approaching 0.1 ms", Physical Review B 86, 100506(R) (2012).

[155] I. M. Pop, K. Geerlings, G. Catelani, R. J. Schoelkopf, L. I. Glazman, and M. H. Devoret, "Coherent suppression of electromagnetic dissipation due to superconducting quasiparticles", Nature 508, 369 (2014).

[156] P. W. Shor, "Scheme for reducing decoherence in quantum computer memory", Physical Review A 52, R2493 (1995).

[157] A. R. Calderbank and P. W. Shor, "Good quantum error-correcting codes exist", Physical Review A 54, 1098 (1996).

[158] A. Steane, "Scheme for reducing decoherence in quantum computer memory", Proceedings of the Royal Society A: Mathematical, Physical and Engineering Sciences 452, 2551 (1996).

[159] E. Knill and R. Laflamme, "Theory of quantum error-correcting codes", Physical Review A 55, 900 (1997).

[160] S. B. Bravyi and A. Y. Kitaev, "Quantum codes on a lattice with boundary", arXiv:quant-ph/9811052 (1998).

[161] A. G. Fowler, M. Mariantoni, J. M. Martinis, and A. N. Cleland, "Surface codes: Towards practical large-scale quantum computation", Physical Review A 86, 032324 (2012). 
[162] M. D. Reed, L. DiCarlo, S. E. Nigg, L. Sun, L. Frunzio, S. M. Girvin, and R. J. Schoelkopf, "Realization of three-qubit quantum error correction with superconducting circuits", Nature 482, 382 (2012).

[163] W. K. Wootters and W. H. Zurek, "A single quantum cannot be cloned", Nature 299, 802 (1982).

[164] D. Dieks, "Communication by EPR devices", Physics Letters A 92, 271 (1982).

[165] K. Lalumiere, J. M. Gambetta, and A. Blais, "Tunable joint measurements in the dispersive regime of cavity QED", Physical Review A 81, 040301(R) (2010).

[166] M. Sandberg, "Fast-tunable resonators and quantum electrical circuits", $\mathrm{PhD}$ thesis (Chalmers University of Technology, Gothenburg, 2009).

[167] M. V. Gustafsson, "Studies of acoustic waves, noise and charge pumping using single-electron devices", $\mathrm{PhD}$ thesis (Chalmers University of Technology, Gothenburg, 2012).

[168] A. Pourkabirian, "Probing quantum and classical noise in nano circuits", PhD thesis (Chalmers University of Technology, Gothenburg, 2014).

[169] D. Leibfried, R. Blatt, C. Monroe, and D. Wineland, "Quantum dynamics of single trapped ions", Reviews of Modern Physics 75, 281 (2003).

[170] T. Monz, P. Schindler, D. Nigg, and R. Blatt, "Quantum information science: experimental implementation with trapped ions", in Quantum Machines: Measurement and Control of Engineered Quantum Systems (Les Houches Session XCVI), edited by M. H. Devoret, B. Huard, R. J. Schoelkopf, and L. F. Cugliandolo (Oxford University Press, 2014).

[171] C. Sayrin, I. Dotsenko, X. Zhou, B. Peaudecerf, T. Rybarczyk, S. Gleyzes, P. Rouchon, M. Mirrahimi, H. Amini, M. Brune, J.-M. Raimond, and S. Haroche, "Real-time quantum feedback prepares and stabilizes photon number states", Nature 477, 73 (2011). 
[172] J.-M. Raimond, "Exploring the quantum world with photons trapped in cavities and Rydberg atoms", in Quantum Machines: Measurement and Control of Engineered Quantum Systems (Les Houches Session XCVI), edited by M. H. Devoret, B. Huard, R. J. Schoelkopf, and L. F. Cugliandolo (Oxford University Press, 2014).

[173] J. Koch, T. M. Yu, J. Gambetta, A. A. Houck, D. I. Schuster, J. Majer, A. Blais, M. H. Devoret, S. M. Girvin, and R. J. Schoelkopf, "Charge-insensitive qubit design derived from the Cooper pair box", Physical Review A 76, 042319 (2007).

[174] E. T. Jaynes and F. W. Cummings, "Comparison of Quantum and Semiclassical Radiation Theories with Application to the Beam Maser", Proceedings of the IEEE 51, 89 (1963).

[175] B. Yurke and J. S. Denker, "Quantum Network Theory", Physical Review A 29, 1419 (1984).

[176] M. H. Devoret, "Quantum fluctuations in electrical circuits", in Quantum Fluctuations: Les Houches Session LXIII, edited by S. Reynaud, E. Giacobino, and J. Zinn-Justin (North Holland, 1997).

[177] L. Tornberg, "Superconducting qubits - measurement, entanglement, and noise", PhD thesis (Chalmers University of Technology, Gothenburg, 2009).

[178] J. R. Johansson, "Quantum mechanics in superconducting circuits and nanomechanical devices", $\mathrm{PhD}$ thesis (Chalmers University of Technology, Gothenburg, 2009).

[179] L. S. Bishop, "Circuit Quantum Electrodynamics", PhD thesis (Yale University, New Haven, 2010).

[180] H. Goldstein, Classical Mechanics, 2nd ed. (Addison-Wesley Publishing Company, Inc., 1980).

[181] D. M. Pozar, Microwave Engineering, 3rd ed. (John Wiley \& Sons, Inc., 2005).

[182] R. E. Collin, Foundations for Microwave Engineering, 2nd ed. (WileyIEEE Press, 2001).

[183] B. Peropadre, J. Lindkvist, I.-C. Hoi, C. M. Wilson, J. J. GarciaRipoll, P. Delsing, and G. Johansson, "Scattering of coherent states on a single artificial atom", New Journal of Physics 15, 035009 (2013). 
[184] J. M. Martinis, M. H. Devoret, and J. Clarke, "Energy-Level Quantization in the Zero-Voltage State of a Current-Biased Josephson Junction", Physical Review Letters 55, 1543 (1985).

[185] Y. Makhlin, G. Schön, and A. Shnirman, "Josephson-junction qubits with controlled couplings", Nature 398, 305 (1999).

[186] Y. Nakamura, Y. A. Pashkin, and J. S. Tsai, "Coherent control of macroscopic quantum states in a single-Cooper-pair box", Nature 398, 786 (1999).

[187] D. Vion, A. Aassime, A. Cottet, P. Joyez, H. Pothier, C. Urbina, D. Esteve, and M. H. Devoret, "Manipulating the Quantum State of an Electrical Circuit", Science 296, 886 (2002).

[188] V. E. Manucharyan, J. Koch, L. I. Glazman, and M. H. Devoret, "Fluxonium: Single Cooper-Pair Circuit Free of Charge Offsets", Science 326, 113 (2009).

[189] S. J. Srinivasan, A. J. Hoffman, J. M. Gambetta, and A. A. Houck, "Tunable Coupling in Circuit Quantum Electrodynamics Using a Superconducting Charge Qubit with a V-Shaped Energy Level Diagram", Physical Review Letters 106, 083601 (2011).

[190] F. Lecocq, I. M. Pop, I. Matei, E. Dumur, A. K. Feofanov, C. Naud, W. Guichard, and O. Buisson, "Coherent Frequency Conversion in a Superconducting Artificial Atom with Two Internal Degrees of Freedom", Physical Review Letters 108, 107001 (2012).

[191] V. Bouchiat, D. Vion, P. Joyez, D. Esteve, and M. H. Devoret, "Quantum Coherence with a Single Cooper Pair", Physica Scripta T76, 165 (1998).

[192] L. DiCarlo, M. D. Reed, L. Sun, B. R. Johnson, J. M. Chow, J. M. Gambetta, L. Frunzio, S. M. Girvin, M. H. Devoret, and R. J. Schoelkopf, "Preparation and measurement of three-qubit entanglement in a superconducting circuit", Nature 467, 574 (2010).

[193] A. A. Abdumalikov Jr, J. M. Fink, K. Juliusson, M. Pechal, S. Berger, A. Wallraff, and S. Filipp, "Experimental realization of nonAbelian non-adiabatic geometric gates", Nature 496, 482 (2013).

[194] A. Muthukrishnan and C. R. Stroud, Jr., "Multivalued logic gates for quantum computation", Physical Review A 62, 052309 (2000). 
[195] S. S. Bullock, D. P. O'Leary, and G. K. Brennen, "Asymptotically Optimal Quantum Circuits for $d$-Level Systems", Physical Review Letters 94, 230502 (2005).

[196] B. P. Lanyon, M. Barbieri, M. P. Almeida, T. Jennewein, T. C. Ralph, K. J. Resch, G. J. Pryde, J. L. O'Brien, A. Gilchrist, and A. G. White, "Simplifying quantum logic using higher-dimensional Hilbert spaces", Nature Physics 5, 134 (2009).

[197] M. Neeley, M. Ansmann, R. C. Bialczak, M. Hofheinz, E. Lucero, A. D. O'Connell, D. Sank, H. Wang, J. Wenner, A. N. Cleland, M. R. Geller, and J. M. Martinis, "Emulation of a Quantum Spin with a Superconducting Phase Qudit", Science 325, 722 (2009).

[198] R. Bianchetti, S. Filipp, M. Baur, J. M. Fink, C. Lang, L. Steffen, M. Boissonneault, A. Blais, and A. Wallraff, "Control and Tomography of a Three Level Superconducting Artificial Atom", Physical Review Letters 105, 223601 (2010).

[199] M. J. Peterer, S. J. Bader, X. Jin, F. Yan, A. Kamal, T. Gudmundsen, P. J. Leek, T. P. Orlando, W. D. Oliver, and S. Gustavsson, "Coherence and Decay of Higher Energy Levels of a Superconducting Transmon Qubit", arXiv:1409.6031 (2014).

[200] R. Barends et al., "Coherent Josephson Qubit Suitable for Scalable Quantum Integrated Circuits", Physical Review Letters 111, 080502 (2013).

[201] Y. Chen et al., "Qubit architecture with high coherence and fast tunable coupling", arXiv:1402.7367, to appear in Physical Review Letters (2014).

[202] Lord Rayleigh, "On Waves Propagated along the Plane Surface of an Elastic Solid", Proceedings of the London Mathematical Society s1-17, 4 (1885).

[203] R. White and F. Voltmer, "Direct piezoelectric coupling to surface elastic waves", Applied Physics Letters 7, 314 (1965).

[204] M. V. Gustafsson, P. V. Santos, G. Johansson, and P. Delsing, "Local probing of propagating acoustic waves in a gigahertz echo chamber", Nature Physics 8, 338 (2012). 
[205] G. Kirchmair, B. Vlastakis, Z. Leghtas, S. E. Nigg, H. Paik, E. Ginossar, M. Mirrahimi, L. Frunzio, S. M. Girvin, and R. J. Schoelkopf, "Observation of quantum state collapse and revival due to the singlephoton Kerr effect", Nature 495, 205 (2013).

[206] I. I. Rabi, "Space Quantization in a Gyrating Magnetic Field", Physical Review 51, 652 (1937).

[207] F. Bloch and A. Siegert, "Magnetic Resonance for Nonrotating Fields", Physical Review 57, 522 (1940).

[208] H.-I. Yoo and J. H. Eberly, "Dynamical theory of an atom with two or three levels interacting with quantized cavity fields", Physics Reports 118, 239 (1985).

[209] D. Braak, "Integrability of the Rabi Model", Physical Review Letters 107, 100401 (2011).

[210] M. Tavis and F. W. Cummings, "Exact Solution for an $N$-MoleculeRadiation-Field Hamiltonian", Physical Review 170, 379 (1968).

[211] P. L. Knight and P. W. Milonni, "The Rabi frequency in optical spectra", Physics Reports 66, 21 (1980).

[212] M. Boissonneault, J. M. Gambetta, and A. Blais, "Dispersive regime of circuit QED: Photon-dependent qubit dephasing and relaxation rates", Physical Review A 79, 013819 (2009).

[213] N. Boulant, G. Ithier, P. Meeson, F. Nguyen, D. Vion, D. Esteve, I. Siddiqi, R. Vijay, C. Rigetti, F. Pierre, and M. Devoret, "Quantum nondemolition readout using a Josephson bifurcation amplifier", Physical Review B 76, 014525 (2007).

[214] B. R. Johnson, M. D. Reed, A. A. Houck, D. I. Schuster, L. S. Bishop, E. Ginossar, J. M. Gambetta, L. DiCarlo, L. Frunzio, S. M. Girvin, and R. J. Schoelkopf, "Quantum non-demolition detection of single microwave photons in a circuit", Nature Physics 6, 663 (2010).

[215] J. Gambetta, A. Blais, M. Boissonneault, A. A. Houck, D. I. Schuster, and S. M. Girvin, "Quantum trajectory approach to circuit QED: Quantum jumps and the Zeno effect", Physical Review A 77, 012112 (2008).

[216] L. Tornberg and G. Johansson, "High-fidelity feedback-assisted parity measurement in circuit QED", Physical Review A 82, 012329 (2010). 
[217] M. Devoret, S. Girvin, and R. Schoelkopf, "Circuit-QED: How strong can the coupling between a Josephson junction atom and a transmission line resonator be?", Annalen der Physik 16, 767 (2007).

[218] J. Bourassa, J. M. Gambetta, A. A. Abdumalikov, Jr., O. Astafiev, Y. Nakamura, and A. Blais, "Ultrastrong coupling regime of cavity QED with phase-biased flux qubits", Physical Review A 80, 032109 (2009).

[219] B. Peropadre, P. Forn-Díaz, E. Solano, and J. J. García-Ripoll, "Switchable Ultrastrong Coupling in Circuit QED", Physical Review Letters 105, 023601 (2010).

[220] J. Bourassa, F. Beaudoin, J. M. Gambetta, and A. Blais, "Josephsonjunction-embedded transmission-line resonators: From Kerr medium to in-line transmon", Physical Review A 86, 013814 (2012).

[221] G. Günter, A. A. Anappara, J. Hees, A. Sell, G. Biasiol, L. Sorba, S. D. Liberato, C. Ciuti, A. Tredicucci, A. Leitenstorfer, and R. Huber, "Sub-cycle switch-on of ultrastrong light-matter interaction", Nature 458, 178 (2009).

[222] T. Schwartz, J. A. Hutchison, C. Genet, and T. W. Ebbesen, "Reversible Switching of Ultrastrong Light-Molecule Coupling", Physical Review Letters 106, 196405 (2011).

[223] T. Niemczyk, F. Deppe, H. Huebl, E. P. Menzel, F. Hocke, M. J. Schwarz, J. J. Garcia-Ripoll, D. Zueco, T. Hümmer, E. Solano, A. Marx, and R. Gross, "Circuit quantum electrodynamics in the ultrastrong-coupling regime", Nature Physics 6, 772 (2010).

[224] P. Forn-Díaz, J. Lisenfeld, D. Marcos, J. J. García-Ripoll, E. Solano, C. J. P. M. Harmans, and J. E. Mooij, "Observation of the BlochSiegert Shift in a Qubit-Oscillator System in the Ultrastrong Coupling Regime", Physical Review Letters 105, 237001 (2010).

[225] G. Scalari, C. Maissen, D. Turcinková, D. Hagenmüller, S. De Liberato, C. Ciuti, C. Reichl, D. Schuh, W. Wegscheider, M. Beck, and J. Faist, "Ultrastrong Coupling of the Cyclotron Transition of a 2D Electron Gas to a THz Metamaterial", Science 335, 1323 (2012).

[226] C. Maissen, G. Scalari, F. Valmorra, S. Cibella, R. Leoni, C. Reichl, C. Charpentier, W. Wegscheider, M. Beck, and J. Faist, "Ultrastrong Coupling in the Near-field of Complementary Split Ring Resonators", arXiv:1408.3547 (2014). 
[227] M. Goryachev, W. G. Farr, D. L. Creedon, Y. Fan, M. Kostylev, and M. E. Tobar, "High-Cooperativity Cavity QED with Magnons at Microwave Frequencies", Physical Review Applied 2, 054002 (2014).

[228] J.-T. Shen and S. Fan, "Coherent Single Photon Transport in a OneDimensional Waveguide Coupled with Superconducting Quantum Bits", Physical Review Letters 95, 213001 (2005).

[229] D. E. Chang, A. S. Sørensen, E. A. Demler, and M. D. Lukin, "A single-photon transistor using nanoscale surface plasmons", Nature Physics 3, 807 (2007).

[230] J.-T. Shen and S. Fan, "Strongly Correlated Two-Photon Transport in a One-Dimensional Waveguide Coupled to a Two-Level System", Physical Review Letters 98, 153003 (2007).

[231] J.-T. Shen and S. Fan, "Strongly correlated multiparticle transport in one dimension through a quantum impurity", Physical Review A 76, 062709 (2007).

[232] P. Longo, P. Schmitteckert, and K. Busch, "Few-Photon Transport in Low-Dimensional Systems: Interaction-Induced Radiation Trapping", Physical Review Letters 104, 023602 (2010).

[233] D. Witthaut and A. S. Sørensen, "Photon scattering by a three-level emitter in a one-dimensional waveguide", New Journal of Physics 12, 043052 (2010).

[234] H. Zheng, D. J. Gauthier, and H. U. Baranger, "Waveguide QED: Many-body bound-state effects in coherent and Fock-state scattering from a two-level system", Physical Review A 82, 063816 (2010).

[235] Y. Wang, J. Minar, L. Sheridan, and V. Scarani, "Efficient excitation of a two-level atom by a single photon in a propagating mode", Physical Review A 83, 063842 (2011).

[236] D. Roy, "Two-Photon Scattering by a Driven Three-Level Emitter in a One-Dimensional Waveguide and Electromagnetically Induced Transparency", Physical Review Letters 106, 053601 (2011).

[237] P. Kolchin, R. F. Oulton, and X. Zhang, "Nonlinear Quantum Optics in a Waveguide: Distinct Single Photons Strongly Interacting at the Single Atom Level", Physical Review Letters 106, 113601 (2011).

[238] H. Zheng, D. J. Gauthier, and H. U. Baranger, "Cavity-Free Photon Blockade Induced by Many-Body Bound States", Physical Review Letters 107, 223601 (2011). 
[239] H. Zheng, D. J. Gauthier, and H. U. Baranger, "Strongly correlated photons generated by coupling a three- or four-level system to a waveguide", Physical Review A 85, 043832 (2012).

[240] E. Rephaeli and S. Fan, "Stimulated Emission from a Single Excited Atom in a Waveguide", Physical Review Letters 108, 143602 (2012).

[241] R. H. Lehmberg, "Radiation from an N-Atom System. I. General Formalism", Physical Review A 2, 883 (1970).

[242] Z. Ficek and B. C. Sanders, "Quantum beats in two-atom resonance fluorescence", Physical Review A 41, 359 (1990).

[243] G. Lenz and P. Meystre, "Resonance fluorescence from two identical atoms in a standing-wave field", Physical Review A 48, 3365 (1993).

[244] Z. Ficek and R. Tanaś, "Entangled states and collective nonclassical effects in two-atom systems", Physics Reports 372, 369 (2002).

[245] G. Ordonez and S. Kim, "Complex collective states in a one-dimensional two-atom system", Physical Review A 70, 032702 (2004).

[246] F. L. Kien, S. D. Gupta, K. P. Nayak, and K. Hakuta, "Nanofibermediated radiative transfer between two distant atoms", Physical Review A 72, 063815 (2005).

[247] A. Gonzalez-Tudela, D. Martin-Cano, E. Moreno, L. Martin-Moreno, C. Tejedor, and F. J. Garcia-Vidal, "Entanglement of Two Qubits Mediated by One-Dimensional Plasmonic Waveguides", Physical Review Letters 106, 020501 (2011).

[248] D. Zueco, J. J. Mazo, E. Solano, and J. J. García-Ripoll, "Microwave photonics with Josephson junction arrays: Negative refraction index and entanglement through disorder", Physical Review B 86, 024503 (2012).

[249] K. Lalumière, B. C. Sanders, A. F. van Loo, A. Fedorov, A. Wallraff, and A. Blais, "Input-output theory for waveguide QED with an ensemble of inhomogeneous atoms", Physical Review A 88, 043806 (2013).

[250] H. Zheng and H. U. Baranger, "Persistent Quantum Beats and LongDistance Entanglement from Waveguide-Mediated Interactions", Physical Review Letters 110, 113601 (2013). 
[251] M. Laakso and M. Pletyukhov, "Scattering of Two Photons from Two Distant Qubits: Exact Solution", Physical Review Letters 113, 183601 (2014).

[252] B. Peropadre, D. Zueco, D. Porras, and J. J. Garcia-Ripoll, "Nonequilibrium and nonperturbative dynamics of ultrastrong coupling in open lines", Physical Review Letters 111, 243602 (2013).

[253] E. Sanchez-Burillo, D. Zueco, J. J. Garcia-Ripoll, and L. MartinMoreno, "Scattering in the ultrastrong regime: nonlinear optics with one photon", arXiv:1406.5779 (2014).

[254] H. P. Breuer and F. Petruccione, The theory of Open Quantum Systems (Oxford University Press, 2002).

[255] H. J. Carmichael, Statistical Methods in Quantum Optics 1 (SpringerVerlag, 1999).

[256] G. Lindblad, "On the generators of quantum dynamical semigroups", Communications in Mathematical Physics 48, 119 (1976).

[257] G. S. Agarwal, "Rotating-Wave Approximation and Spontaneous Emission", Physical Review A 7, 1195 (1973).

[258] G. S. Agarwal, "Quantum statistical theories of spontaneous emission and their relation to other approaches", Springer Tracts in Modern Physics 70, 1 (1974).

[259] C. W. Gardiner and M. J. Collett, "Input and output in damped quantum systems: Quantum stochastic differential equations and the master equation", Physical Review A 31, 3761 (1985).

[260] R. L. Hudson and K. R. Parthasarathy, "Quantum Itō's Formula and Stochastic Evolutions", Communications in Mathematical Physics 93, 301 (1984).

[261] L. Bouten, R. van Handel, and M. R. James, "An introduction to quantum filtering", SIAM Journal on Control and Optimization 46, 2199 (2007).

[262] J. Kerckhoff, "Quantum Engineering with Quantum Optics", PhD thesis (Stanford University, Stanford, 2011).

[263] B. Q. Baragiola, R. L. Cook, A. M. Brańczyk, and J. Combes, "Nphoton wave packets interacting with an arbitrary quantum system", Physical Review A 86, 013811 (2012). 
[264] B. Q. Baragiola, "Open systems dynamics for propagating quantum fields", PhD thesis (University of New Mexico, Albuquerque, 2014).

[265] K. M. Gheri, K. Ellinger, T. Pellizzari, and P. Zoller, "PhotonWavepackets as Flying Quantum Bits", Fortschritte der Physik 46, 401 (1998).

[266] J. E. Gough, M. R. James, H. I. Nurdin, and J. Combes, "Quantum filtering for systems driven by fields in single-photon states or superposition of coherent states", Physical Review A 86, 043819 (2012).

[267] R. Loudon, The Quantum Theory of Light, 3rd ed. (Oxford University Press, 2000).

[268] K. J. Blow, R. Loudon, S. J. D. Phoenix, and T. J. Shepherd, "Continuum fields in quantum optics", Physical Review A 42, 4102 (1990).

[269] J. E. Gough, M. R. James, and H. I. Nurdin, "Quantum master equation and filter for systems driven by fields in a single photon state", in 50th IEEE Conference on Decision and Control and European Control Conference (2011), p. 5570.

[270] H. J. Carmichael, "Quantum Trajectory Theory for Cascaded Open Systems", Physical Review Letters 70, 2273 (1993).

[271] H. M. Wiseman, "Quantum Trajectories and Feedback", PhD thesis (University of Queensland, Brisbane, 1994).

[272] H. M. Wiseman and G. J. Milburn, Quantum Measurement and Control (Cambridge University Press, 2010).

[273] H. J. Carmichael, Statistical Methods in Quantum Optics 2 (SpringerVerlag, 2008).

[274] C. Eichler, "Experimental Characterization of Quantum Microwave Radiation and its Entanglement with a Superconducting Qubit", PhD thesis (ETH, Zürich, 2013).

[275] M. da Silva, D. Bozyigit, A. Wallraff, and A. Blais, "Schemes for the observation of photon correlation functions in circuit QED with linear detectors", Physical Review A 82, 043804 (2010).

[276] M. A. Castellanos-Beltran, K. D. Irwin, G. C. Hilton, L. R. Vale, and K. W. Lehnert, "Amplification and squeezing of quantum noise with a tunable Josephson metamaterial", Nature Physics 4, 929 (2008). 
[277] M. A. Castellanos-Beltran, "Development of a Josephson Parametric Amplifier for the Preparation and Detection of Nonclassical States of Microwave Fields", PhD thesis (University of Colorado, Boulder, 2010).

[278] F. Mallet, M. A. Castellanos-Beltran, H. S. Ku, S. Glancy, E. Knill, K. D. Irwin, G. C. Hilton, L. R. Vale, and K. W. Lehnert, "Quantum State Tomography of an Itinerant Squeezed Microwave Field", Physical Review Letters 106, 220502 (2011).

[279] R. Vijay, C. Macklin, D. H. Slichter, S. J. Weber, K. W. Murch, R. Naik, A. N. Korotkov, and I. Siddiqi, "Stabilizing Rabi oscillations in a superconducting qubit using quantum feedback", Nature 490, 77 (2012).

[280] K. W. Murch, S. J. Weber, C. Macklin, and I. Siddiqi, "Observing single quantum trajectories of a superconducting quantum bit", Nature 502, 211 (2013).

[281] F. D. O. Schackert, "A Practical Quantum-Limited Parametric Amplifier Based on the Josephson Ring Modulator", $\mathrm{PhD}$ thesis (Yale University, New Haven, 2013).

[282] E. Flurin, "The Josephson mixer - A swiss army knife for microwave quantum optics", $\mathrm{PhD}$ thesis (École Normale supérieure, Paris, 2014).

[283] E. Platen and N. Bruti-Liberati, Numerical Solution of Stochastic Differential Equations with Jumps in Finance (Springer-Verlag, 2010).

[284] R. Kubo, "Statistical-Mechanical Theory of Irreversible Processes. I. General Theory and Simple Applications to Magnetic and Conduction Problems", Journal of the Physical Society of Japan 12, 570 (1957).

[285] I. Rau, G. Johansson, and A. Shnirman, "Cavity quantum electrodynamics in superconducting circuits: Susceptibility at elevated temperatures", Physical Review B 70, 054521 (2004).

[286] C. W. Gardiner, "Driving a quantum system with the output field from another driven quantum system", Physical Review Letters 70, 2269 (1993). 
[287] J. Gough and M. R. James, "Quantum Feedback Networks: Hamiltonian Formulation", Communications in Mathematical Physics 287, 1109 (2009).

[288] J. Gough and M. R. James, "The Series Product and Its Application to Quantum Feedforward and Feedback Networks", IEEE Transactions on Automatic Control 54, 2530 (2009).

[289] N. Tezak, A. Niederberger, D. S. Pavlichin, G. Sarma, and H. Mabuchi, "Specification of photonic circuits using Quantum Hardware Description Language", Philosophical Transactions of the Royal Society A: Mathematical, Physical and Engineering Sciences 370, 5270 (2012).

[290] J. Kerckhoff and K. W. Lehnert, "Superconducting Microwave Multivibrator Produced by Coherent Feedback", Physical Review Letters 109, 153602 (2012).

[291] D. P. DiVincenzo and F. Solgun, "Multi-qubit parity measurement in circuit quantum electrodynamics", New Journal of Physics 15, 075001 (2013).

[292] L. Tornberg, S. Barzanjeh, and D. P. DiVincenzo, "Stochastic-masterequation analysis of optimized three-qubit nondemolition parity measurements", Physical Review A 89, 032314 (2014).

[293] S. E. Nigg and S. M. Girvin, "Stabilizer Quantum Error Correction Toolbox for Superconducting Qubits", Physical Review Letters 110, 243604 (2013).

[294] G. de Lange, D. Ristè, M. J. Tiggelman, C. Eichler, L. Tornberg, G. Johansson, A. Wallraff, R. N. Schouten, and L. DiCarlo, "Reversing Quantum Trajectories with Analog Feedback", Physical Review Letters 112, 080501 (2014).

[295] B. Fan, G. Johansson, J. Combes, G. J. Milburn, and T. M. Stace, "Nonabsorbing high-efficiency counter for itinerant microwave photons", Physical Review A 90, 035132 (2014).

[296] W. E. Shanks, D. L. Underwood, and A. A. Houck, "A scanning transmon qubit for strong coupling circuit quantum electrodynamics", Nature Communications 4, 1991 (2013). 
[297] M. Lee, J. Kim, W. Seo, H.-G. Hong, Y. Song, R. R. Dasari, and K. An, "Three-dimensional imaging of cavity vacuum with single atoms localized by a nanohole array", Nature Communications $\mathbf{5}$, 3441 (2014).

[298] M. V. Gustafsson, T. Aref, A. F. Kockum, M. K. Ekström, R. Manenti, P. Leek, G. Johansson, and P. Delsing, "Using phonons for quantum information", to appear in Superconducting devices in quantum optics, edited by R. Hadfield and G. Johansson (SpringerVerlag, 2015).

[299] J. Bochmann, A. Vainsencher, D. D. Awschalom, and A. N. Cleland, "Nanomechanical coupling between microwave and optical photons", Nature Physics 9, 712 (2013).

[300] R. W. Andrews, R. W. Peterson, T. P. Purdy, K. Cicak, R. W. Simmonds, C. A. Regal, and K. W. Lehnert, "Bidirectional and efficient conversion between microwave and optical light", Nature Physics 10, 321 (2014).

[301] S. J. M. Habraken, K. Stannigel, M. D. Lukin, P. Zoller, and P. Rabl, "Continuous mode cooling and phonon routers for phononic quantum networks", New Journal of Physics 14, 115005 (2012).

[302] U. Dorner and P. Zoller, "Laser-driven atoms in half-cavities", Physical Review A 66, 023816 (2002).

[303] J. Zhang, Y.-X. Liu, R.-B. Wu, K. Jacobs, and F. Nori, "NonMarkovian quantum input-output networks", Physical Review A 87, 032117 (2013).

[304] J. Johansson, P. Nation, and F. Nori, "QuTiP: An open-source Python framework for the dynamics of open quantum systems", Computer Physics Communications 183, 1760 (2012).

[305] J. Johansson, P. Nation, and F. Nori, "QuTiP 2: A Python framework for the dynamics of open quantum systems", Computer Physics Communications 184, 1234 (2013).

[306] J. Koch, A. A. Houck, K. L. Hur, and S. M. Girvin, "Time-reversalsymmetry breaking in circuit-QED-based photon lattices", Physical Review A 82, 043811 (2010). 
[307] J. Kerckhoff, H. Nurdin, D. S. Pavlichin, and H. Mabuchi, "Designing quantum memories with embedded control: photonic circuits for autonomous quantum error correction", Physical Review Letters 105, 040502 (2010).

[308] J. Kerckhoff, D. S. Pavlichin, H. Chalabi, and H. Mabuchi, "Design of nanophotonic circuits for autonomous subsystem quantum error correction", New Journal of Physics 13, 055022 (2011).

[309] S. Schmidt and J. Koch, "Circuit QED lattices: Towards quantum simulation with superconducting circuits", Annalen der Physik 525, 395 (2013).

[310] J. R. Schrieffer and P. A. Wolff, "Relation between the Anderson and Kondo Hamiltonians", Physical Review 149, 491 (1966). 